\title{
Gravity duals of supersymmetric gauge theories on three-manifolds
}

\author{
Daniel Farquet, ${ }^{a}$ Jakob Lorenzen, ${ }^{b}$ Dario Martelli ${ }^{b}$ and James Sparks ${ }^{a}$ \\ ${ }^{a}$ Mathematical Institute, University of Oxford, \\ Radcliffe Observatory Quarter, Woodstock Road, Oxford OX2 6GG, U.K. \\ ${ }^{b}$ Department of Mathematics, King's College London, \\ The Strand, London WC2R 2LS, U.K. \\ E-mail: farquet@maths.ox.ac.uk, jakob.lorenzen@kcl.ac.uk, \\ dario.martelli@kcl.ac.uk, sparks@maths.ox.ac.uk
}

ABSTRACT: We study gravity duals to a broad class of $\mathcal{N}=2$ supersymmetric gauge theories defined on a general class of three-manifold geometries. The gravity backgrounds are based on Euclidean self-dual solutions to four-dimensional gauged supergravity. As well as constructing new examples, we prove in general that for solutions defined on the four-ball the gravitational free energy depends only on the supersymmetric Killing vector, finding a simple closed formula when the solution has $\mathrm{U}(1) \times \mathrm{U}(1)$ symmetry. Our result agrees with the large $N$ limit of the free energy of the dual gauge theory, computed using localization. This constitutes an exact check of the gauge/gravity correspondence for a very broad class of gauge theories with a large $N$ limit, defined on a general class of background three-manifold geometries.

KEYWORDS: Gauge-gravity correspondence, Supersymmetric gauge theory

ARXIV EPRINT: 1404.0268 


\section{Contents}

1 Introduction 2

2 Local geometry of self-dual solutions 4

2.1 Local form of the solution 5

2.2 Conformal Kähler metric 6

2.3 Killing spinor: sufficiency

3 Asymptotically locally AdS solutions $\quad 9$

$\begin{array}{ll}3.1 \text { Conformal boundary at } y=0 & 10\end{array}$

$\begin{array}{lll}3.2 & \text { Boundary Killing spinor } & 12\end{array}$

$\begin{array}{lll}3.3 & \text { Non-singular gauge } & 13\end{array}$

$\begin{array}{ll}3.4 & \text { Global conformal Kähler structure } \\ \end{array}$

$\begin{array}{ll}3.5 \text { Toric formulae } & 18\end{array}$

4 Holographic free energy $\quad 19$

$\begin{array}{ll}4.1 \text { General formulae } & 19\end{array}$

4.2 The four-ball 20

$\begin{array}{ll}4.3 & \text { Index theory formulae } \\ \end{array}$

5 Examples $\quad 25$

$\begin{array}{lll}5.1 & \mathrm{AdS}_{4} & 25\end{array}$

$\begin{array}{lll}5.2 & \text { Taub-NUT-AdS } & \\ 5.3 & \text { Plebansk-Demian } & 27\end{array}$

5.3 Plebanski-Demianski 30

5.4 Infinite parameter generalization 31

6 Conclusions 33

A Spin connection of the Kähler metric $\quad 35$

B Weyl transformations of the boundary 36

C Toric self-dual Einstein metrics on the four-ball 39

$\begin{array}{lll}\text { C.1 Local form of the metrics and instanton } & 39\end{array}$

C.2 $m$-pole solutions 41

C.3 $\mathrm{AdS}_{4}$ from 2-pole solution 43

C.4 Plebanski-Demianski from 3-pole solution 46

$\begin{array}{lll}\text { C.5 General instanton on Plebanski-Demianski } & 55\end{array}$

C.6 Taub-NUT-AdS 4 as a limit of Plebanski-Demianski 57 


\section{Introduction}

Exact results in quantum field theories are rare and for some time the gauge/gravity duality [1-3] has been a main tool for obtaining such results in a growing variety of situations. More recently, it has been appreciated that exact non-perturbative computations can be performed in certain supersymmetric field theories defined on curved Riemannian manifolds, using the technique of localization [4]. On the one hand, this has motivated the systematic study of rigid supersymmetry in curved space [5], and on the other hand it has prompted the exploration of the gauge/gravity duality in situations when the boundary supersymmetric field theories are defined on non-trivial curved manifolds. This programme has been initiated in [6], where a simple (Euclidean) supersymmetric solution of fourdimensional minimal gauged supergravity was proposed as the dual to three-dimensional supersymmetric Chern-Simons quiver theories defined on a squashed three-sphere (ellipsoid), for which the exact partition function had been computed previously in [7]. Generalizations have been discussed by some of the authors in [8-10]. Further examples of four-dimensional gravity solutions with curved boundary, where in the dual field theory the path integral can be computed exactly using localization, have been discussed in $[11,12]$. In this case, the exactly calculable quantity on both sides of the duality is the so-called supersymmetric Rényi entropy [13], which is a simple modification of the partition function on the ellipsoid [7] (see also [6]). In five bulk dimensions a supersymmetric solution, where holographic computations have been compared with exact four-dimensional results in $\mathcal{N}=1$ SCFTs, has been recently constructed in [14], while in [15] the gravity dual to supersymmetric gauge theories on a squashed five-sphere has been constructed in Romans $F(4)$ gauged supergravity in six dimensions, and the holographic free energy and BPS Wilson loops successfully matched to localization computations in five dimensions. Gravity solutions dual to exact localization results have also been discussed in [16] (for three-dimensional $\mathcal{N}=2$ theories on $S^{3}$ ) and in [17-19] (for four-dimensional $\mathcal{N}=2^{*}$ theories on $S^{4}$ ). These, however, have conformally flat boundaries.

Using localization, the partition function $Z$ of a large class of $\mathcal{N}=2$ three-dimensional Chern-Simons theories defined on a general manifold with three-sphere topology was computed explicitly in [20]. This has provided a unified understanding of all previous localization computations on deformed three-spheres [6, 7, 13, 21], and has shown that the partition function on these manifolds depends only on a single parameter $b_{1} / b_{2}$, related to a choice of almost contact structure. ${ }^{1}$ Specifically, for a general toric metric on the three-sphere, the real numbers $b_{1}, b_{2}$ specify a choice of Killing vector $K$ in the torus of isometries. For a broad class of Chern-Simons quiver theories, the large $N$ limit of the free energy $\mathcal{F}=-\log |Z|$ can be computed using saddle points methods [6], giving the general result

$$
\lim _{N \rightarrow \infty} \mathcal{F}_{\frac{b_{1}}{b_{2}}}=\frac{1}{4}\left(\sqrt{\left|\frac{b_{1}}{b_{2}}\right|}+\sqrt{\left|\frac{b_{2}}{b_{1}}\right|}\right)^{2} \mathcal{F}_{1},
$$

where $\mathcal{F}_{1}$ is the large $N$ limit of the free energy on the round three-sphere, scaling with $N^{3 / 2}$ [23].

\footnotetext{
${ }^{1}$ This fact has been recovered independently in [22], using different methods.
} 
On the gravity side this yields a universal prediction for the holographically renormalized on-shell action of the corresponding supergravity solutions. Indeed, the on-shell action of the solutions of $[6,8,9]$, and [10] reproduced this formula, for certain choices of metrics and background gauge fields. More precisely, these are all supersymmetric solutions of minimal four-dimensional gauged supergravity in Euclidean signature, and comprise a negatively curved Einstein anti-self-dual metric on the four-ball, ${ }^{2}$ with a specific choice of gauge field with anti-self-dual curvature, that we refer to as an instanton. The result of [20] raises two questions: 1) given an arbitrary (toric) metric on the three-sphere, with a background gauge field satisfying the rigid Killing spinor equations [24, 25], can one construct a dual supegravity solution? 2) Assuming such a supergravity solution exists, can one compute the corresponding holographic free energy and show that it matches (1.1)?

The purpose of this paper is to address these two questions. Working in the context of minimal gauged supergravity, and assuming an ansatz that the solutions are anti-self-dual and have the topology of the ball, we will be able to provide rather general answers to both these questions. In the concluding section we will discuss the possibility of extending our results beyond the class of solutions considered in this paper.

Regarding the first question, we will show that given a (non-singular) anti-self-dual metric on the ball with $\mathrm{U}(1)^{2}$ isometry, and a choice of an arbitrary Killing vector therein, we can construct a (non-singular) instanton configuration, such that together these give a smooth supersymmetric solution of minimal gauged supergravity. Moreover, assuming this metric is asymptotically locally (Euclidean) AdS, we will show that on the conformal boundary the four-dimensional solution reduces to a three-dimensional geometry solving the rigid Killing spinor equations of [24, 25], in the form presented in [20]. We will illustrate this construction through several examples, including previously known as well as new solutions. We will also discuss how all the examples that we will present can be understood as arising from an infinite-dimensional family of explicit " $m$-pole" metrics [26].

We will be able to answer the second question, regarding the computation of the holographic free energy, independently of the details of a specific solution. Namely, assuming only that a smooth solution with given boundary conditions exists, we will show that the holographically renormalized on-shell action takes the form

$$
I=\frac{\pi}{2 G_{4}} \cdot \frac{\left(\left|b_{1}\right|+\left|b_{2}\right|\right)^{2}}{4\left|b_{1} b_{2}\right|}
$$

precisely matching the large $N$ field theory prediction from localization (1.1)! We emphasize that (1.2) will be derived without reference to a specific solution, and that it receives nonzero contributions from the boundary (as expected), as well as from the bulk, specifically from the "centre" of the ball. This latter contribution may be understood as arising from the fixed point of the torus action, and can be determined from a fixed point theorem, using the Berline-Vergne formula. We will also present formulas relating the renormalized on-

\footnotetext{
${ }^{2}$ References [8] and [9] also discuss several solutions with topology different from the four-ball; however, currently the precise field theory constructions dual to these remain unknown. In the present paper we will not discuss topologies different from the four-ball.
} 
shell action to topological invariants of the bulk and conformal invariants of the boundary, by using the Atiyah-Patodi-Singer index theorem, which may be of independent interest.

The rest of this paper is organized as follows. In section 2 we discuss the local geometry of Euclidean supersymmetric solutions of minimal four-dimensional gauged supergravity. In section 3 we turn to global and smooth asymptotically locally Euclidean AdS solutions, with the topology of the four-ball. Section 4 contains the derivation of the general formula (1.2) for the holographic free energy. In section 5 we present examples. In section 6 we conclude by discussing possible extensions of this work. Appendices A and B contain details about the geometry, while in appendix $\mathrm{C}$ we present a unified view of all the examples, arising as particular cases of the $m$-pole metrics [26].

\section{Local geometry of self-dual solutions}

The action for the bosonic sector of four-dimensional $\mathcal{N}=2$ gauged supergravity [27] is

$$
I^{\mathrm{SUGRA}}=-\frac{1}{16 \pi G_{4}} \int\left(R+6-F^{2}\right) \sqrt{\operatorname{det} g} \mathrm{~d}^{4} x
$$

where $R$ denotes the Ricci scalar of the four-dimensional metric $g_{\mu \nu}$, we have defined $F^{2} \equiv F_{\mu \nu} F^{\mu \nu}$, and the cosmological constant has been normalized to $\Lambda=-3$. The graviphoton is an Abelian gauge field $A$ with field strength $F=\mathrm{d} A$. The equations of motion derived from (2.1) are

$$
\begin{aligned}
R_{\mu \nu}+3 g_{\mu \nu} & =2\left(F_{\mu}{ }^{\rho} F_{\nu \rho}-\frac{1}{4} F^{2} g_{\mu \nu}\right), \\
\mathrm{d} *_{4} F & =0 .
\end{aligned}
$$

This is simply Einstein-Maxwell theory with a cosmological constant $\Lambda=-3$. Notice that when $F$ is anti-self-dual the right hand side of the Einstein equation in (2.2) is zero, so that the metric $g_{\mu \nu}$ is necessarily Einstein.

A solution is supersymmetric provided it admits a (not identically zero) Dirac spinor $\epsilon$ satisfying the Killing spinor equation

$$
\left(\nabla_{\mu}-\mathrm{i} A_{\mu}+\frac{1}{2} \Gamma_{\mu}+\frac{\mathrm{i}}{4} F_{\nu \rho} \Gamma^{\nu \rho} \Gamma_{\mu}\right) \epsilon=0
$$

This takes the same form as in Lorentzian signature, except that here the gamma matrices generate the Clifford algebra Cliff(4,0) in an orthonormal frame, so $\left\{\Gamma_{\mu}, \Gamma_{\nu}\right\}=2 g_{\mu \nu}$. Notice that we may define the charge conjugate of the spinor $\epsilon$ as $\epsilon^{c} \equiv B \epsilon^{*}$, where $B$ is the charge conjugation matrix satisfying $B^{-1} \Gamma_{\mu} B=\Gamma_{\mu}^{*}, B B^{*}=-1$ and may be chosen to be antisymmetric $B^{T}=-B$ [6]. Then provided the gauge field $A$ is real (as it will be in the present paper) $\epsilon^{c}$ satisfies (2.3) with $A \rightarrow-A$.

In $[28,29]$ the authors studied the local geometry of Euclidean supersymmetric solutions to the above theory for which $F$ is anti-self-dual, ${ }_{4} F=-F$. It follows that the metric $g_{\mu \nu}$ then has anti-self-dual Weyl tensor, and adopting a standard abuse of terminology we 
shall refer to such solutions as "self-dual". ${ }^{3}$ Supersymmetry also equips this background geometry with a Killing vector field $K$. Self-dual Einstein metrics with a Killing vector have a rich geometric structure that has been well-studied (see for example [31]), and are well-known to be related by a Weyl rescaling to a (local) Kähler metric with zero Ricci scalar. Such metrics are described by a solution to a single PDE, known as the Toda equation, and this solution also specifies uniquely the background gauge field $A$. In fact we will show that $F=\mathrm{d} A$ is $\frac{1}{2}$ the Ricci-form of the conformally related Kähler metric, so that $A$ is the natural connection on $\mathcal{K}^{-1 / 2}$, where $\mathcal{K}$ denotes the canonical bundle of the Kähler manifold. Moreover, we will reverse the direction of implication in [28, 29] and show that any self-dual Einstein metric with a choice of Killing vector field admits (locally) a solution to the Killing spinor equation (2.3). This may be constructed from the canonically defined $\operatorname{spin}^{c}$ spinor that exists on any Kähler manifold.

\subsection{Local form of the solution}

In this section we briefly review the local geometry determined in $[28,29]$. The existence of a non-trivial solution to the Killing spinor equation (2.3), together with the ansatz that $F$ is anti-self-dual and real, implies that the metric $g_{\mu \nu}$ is Einstein with anti-self-dual Weyl tensor. There is then a canonically defined local coordinate system in which the metric takes the form

$$
\mathrm{d} s_{\mathrm{SDE}}^{2}=\frac{1}{y^{2}}\left[V^{-1}(\mathrm{~d} \psi+\phi)^{2}+V\left(\mathrm{~d} y^{2}+4 \mathrm{e}^{w} \mathrm{~d} z \mathrm{~d} \bar{z}\right)\right]
$$

where

$$
\begin{aligned}
V & =1-\frac{1}{2} y \partial_{y} w \\
\mathrm{~d} \phi & =\mathrm{i} \partial_{z} V \mathrm{~d} y \wedge \mathrm{d} z-\mathrm{i} \partial_{\bar{z}} V \mathrm{~d} y \wedge \mathrm{d} \bar{z}+2 \mathrm{i} \partial_{y}\left(V \mathrm{e}^{w}\right) \mathrm{d} z \wedge \mathrm{d} \bar{z}
\end{aligned}
$$

and $w=w(y, z, \bar{z})$ satisfies the Toda equation

$$
\partial_{z} \partial_{\bar{z}} w+\partial_{y}^{2} \mathrm{e}^{w}=0
$$

Notice that the function $w$ determines entirely the metric. The two-form $\mathrm{d} \phi$ is easily verified to be closed provided the Toda equation (2.7) is satisfied, implying the existence of a local one-form $\phi$.

The vector $K=\partial_{\psi}$ is a Killing vector field, and arises canonically from supersymmetry as a bilinear $K^{\mu} \equiv \mathrm{i} \epsilon^{\dagger} \Gamma^{\mu} \Gamma_{5} \epsilon$, where $\epsilon$ is the Killing spinor solving (2.3) and $\Gamma_{5} \equiv \Gamma_{0123}$. Notice that the corresponding bilinear in the charge conjugate spinor $\epsilon^{c}$ is $\mathrm{i}\left(\epsilon^{c}\right)^{\dagger} \Gamma^{\mu} \Gamma_{5} \epsilon^{c}=$ $-K^{\mu}$. Thus as in the discussion after equation (2.3) we may change variables to $\tilde{\epsilon}=\epsilon^{c}, \tilde{A}=$ $-A$. In the tilded variables the equations of motion (2.2) and Killing spinor equation (2.3) are identical to the untilded equations, but now $\tilde{A}=-A$ and $\tilde{K}=-K$. Thus the sign of the instanton is correlated with a choice of sign for the supersymmetric Killing vector, with charge conjugation of the spinor changing the signs of both $A$ and $K$.

\footnotetext{
${ }^{3}$ Einstein four-manifolds with anti-self-dual Weyl tensor and non-zero scalar curvature are also sometimes called quaternionic Kähler four-manifolds, with the condition on the Weyl tensor being referred to as halfconformally flat. See, for example, [30].
} 
As we shall see in the next section, the coordinate $y$ determines the conformal factor for the conformally related Kähler metric, and is also the Hamiltonian function for the vector field $K=\partial_{\psi}$ with respect to the associated symplectic form. The graviphoton field is given (in our conventions) by

$$
A=-\frac{1}{4} V^{-1} \partial_{y} w(\mathrm{~d} \psi+\phi)+\frac{\mathrm{i}}{4} \partial_{z} w \mathrm{~d} z-\frac{\mathrm{i}}{4} \partial_{\bar{z}} w \mathrm{~d} \bar{z}
$$

We are of course free to make gauge transformations of $A$, and we stress that (2.8) is in general valid only locally.

Having summarized the results of $[28,29]$, in the next two sections we study this local geometry further. In particular we show that any self-dual Einstein metric with Killing vector $K \equiv \partial_{\psi}$, which then takes the form (2.4), admits a Killing spinor $\epsilon$ solving (2.3), where $A$ is given by (2.8).

\subsection{Conformal Kähler metric}

As already mentioned, every self-dual Einstein four-metric with a Killing vector is conformally related to a scalar-flat Kähler metric. This is given by

$$
\begin{aligned}
\mathrm{d} s_{\text {Kahler }}^{2} & \equiv \mathrm{d} \hat{s}^{2}=y^{2} \mathrm{~d} s_{\text {SDE }}^{2} \\
& =V^{-1}(\mathrm{~d} \psi+\phi)^{2}+V\left(\mathrm{~d} y^{2}+4 \mathrm{e}^{w} \mathrm{~d} z \mathrm{~d} \bar{z}\right) .
\end{aligned}
$$

Introducing an associated local orthonormal frame of one-forms

$$
\hat{e}^{0}=V^{1 / 2} \mathrm{~d} y, \quad \hat{e}^{1}=V^{-1 / 2}(\mathrm{~d} \psi+\phi), \quad \hat{e}^{2}+\mathrm{i} \hat{e}^{3}=2\left(V \mathrm{e}^{w}\right)^{1 / 2} \mathrm{~d} z,
$$

the Kähler form is

$$
\omega=\hat{e}^{01}+\hat{e}^{23}
$$

where we have denoted $\hat{e}^{0} \wedge \hat{e}^{1}=\hat{e}^{01}$, etc. That (2.11) is indeed closed follows immediately from the expression for $\mathrm{d} \phi$ in (2.6). The Kähler form is self-dual with respect to the natural orientation on a Kähler manifold, namely $\hat{e}^{0123}$ above, and it is with respect to this orientation that the curvature $F$ and Weyl tensor are anti-self-dual. We denote the corresponding orthonormal frame for the self-dual Einstein metric (2.4) as $e^{a}=y^{-1} \hat{e}^{a}, a=0,1,2,3$.

Next we introduce the Hodge type $(2,0)$-form

$$
\Omega \equiv\left(\hat{e}^{0}+\mathrm{i} \hat{e}^{1}\right) \wedge\left(\hat{e}^{2}+\mathrm{i} \hat{e}^{3}\right),
$$

and recall that the metric (2.9) is Kähler if and only if

$$
\mathrm{d} \Omega=\mathrm{i} \mathcal{P} \wedge \Omega,
$$

where $\mathcal{P}$ is then the Ricci one-form, with Ricci two-form $\mathcal{R}=\mathrm{d} \mathcal{P}$. It is straightforward to compute $\mathrm{d} \Omega$ for the metric (2.9), and one finds that

$$
\mathcal{P}=2 A,
$$


where $A$ is given by (2.8). Thus the gauge field is the natural connection on $\mathcal{K}^{-1 / 2}$, where $\mathcal{K}$ denotes the canonical line bundle for the Kähler metric. The curvature is correspondingly $F=\mathrm{d} A=\frac{1}{2} \mathcal{R}$, where recall that $\mathcal{R}_{\mu \nu}=\frac{1}{2} \hat{R}_{\mu \nu \rho \sigma} \omega^{\rho \sigma}$ where $\hat{R}_{\mu \nu \rho \sigma}$ denotes the Riemann tensor for the Kähler metric. A computation gives

$$
-2 \mathcal{R} \wedge \omega=\frac{1}{V \mathrm{e}^{w}}\left[\partial_{z} \partial_{\bar{z}} w+\partial_{y}^{2} \mathrm{e}^{w}\right] \hat{e}^{0123},
$$

so that the Kähler metric is indeed scalar flat if the Toda equation holds. Since the Ricci two-form has Hodge type $(1,1)$ and the metric is scalar flat, it follows immediately that $F=$ $\frac{1}{2} \mathcal{R}$ is anti-self-dual. This is because the anti-self-dual two-forms on a Kähler four-manifold are precisely the primitive $(1,1)$-forms (i.e. having zero wedge product with $\omega$, as in (2.15)), so $\Lambda_{-}^{2} \cong \Lambda_{0}^{(1,1)}$. An explicit computation shows that with respect to the frame (2.10)

$$
\begin{aligned}
F= & -\frac{1}{4} \partial_{y}\left[V^{-1} \partial_{y} w\right]\left(\hat{e}^{01}-\hat{e}^{23}\right)+\frac{1}{8 \mathrm{e}^{w / 2}}\left[\mathrm{i}\left(\partial_{z}-\partial_{\bar{z}}\right)\left[V^{-1} \partial_{y} w\right]\left(\hat{e}^{02}+\hat{e}^{13}\right)\right. \\
& \left.-\left(\partial_{z}+\partial_{\bar{z}}\right)\left[V^{-1} \partial_{y} w\right]\left(\hat{e}^{03}-\hat{e}^{12}\right)\right],
\end{aligned}
$$

which is then manifestly anti-self-dual. One can also derive the formula

$$
F=-\left(\frac{1}{2} y \mathrm{~d} K^{b}+y^{2} K^{b} \wedge J K^{b}\right)^{-}
$$

where $K^{b}$ denotes the one-form dual to the Killing vector $K$ (in the self-dual Einstein metric), and $J$ is the complex structure tensor for the Kähler metric (2.9), and a further short computation leads to

$$
F=\left(\frac{1}{y} \mathrm{i} \partial \bar{\partial} y\right)^{-}=\frac{1}{y} \mathrm{i} \partial \bar{\partial} y+\frac{1}{4 y}(\hat{\Delta} y) \omega
$$

where $\bar{\partial}$ denotes the standard operator on a Kähler manifold, the superscript "-" in (2.18) denotes anti-self-dual part, and $\hat{\Delta}$ denotes the scalar Laplacian for the Kähler metric.

Let us note that the Kähler form is explicitly

$$
\omega=\mathrm{d} y \wedge(\mathrm{d} \psi+\phi)+2 \mathrm{i} V \mathrm{e}^{w} \mathrm{~d} z \wedge \mathrm{d} \bar{z} .
$$

Thus $\left.\mathrm{d} y=-\partial_{\psi}\right\lrcorner \omega$, which identifies the coordinate $y$ as the Hamiltonian function for the Killing vector $K=\partial_{\psi}$. Of course, $y^{2}$ is also the conformal factor relating the self-dual Einstein metric to the Kähler metric in (2.9).

\subsection{Killing spinor: sufficiency}

In this section we show that a self-dual Einstein metric with Killing vector $K=\partial_{\psi}$, which necessarily takes the form (2.4), admits a solution to the Killing spinor equation (2.3) with gauge field given by (2.8). The key to this construction is to begin with the canonically defined $\operatorname{spin}^{c}$ spinor that exists on any Kähler manifold.

The positive chirality spin bundle on a Kähler four-manifold takes the form $\mathcal{S}_{+} \cong$ $\mathcal{K}^{1 / 2} \oplus \mathcal{K}^{-1 / 2}$, where $\mathcal{K}$ denotes the canonical bundle. The spin bundle then exists globally 
only if the latter admits a square root, but the $\operatorname{spin}^{c}$ bundle $\mathcal{S}_{+} \otimes \mathcal{K}^{-1 / 2} \cong 1 \oplus \mathcal{K}^{-1}$ always exists globally. In particular the first factor in $\mathcal{S}_{+} \otimes \mathcal{K}^{-1 / 2} \cong 1 \oplus \mathcal{K}^{-1}$ is a trivial complex line bundle, whose sections may be identified with complex-valued functions, and there is always a section $\zeta$ satisfying the $\operatorname{spin}^{c}$ Killing spinor equation

$$
\left(\hat{\nabla}_{\mu}-\frac{\mathrm{i}}{2} \mathcal{P}_{\mu}\right) \zeta=0
$$

Here the hat denotes that we will apply this to the conformal Kähler metric (2.9) in the case at hand, and $\mathcal{P}$ is the Ricci one-form potential we encountered above. The connection term in (2.20) precisely corresponds to twisting the spin bundle $\mathcal{S}_{+}$by $\mathcal{K}^{-1 / 2}$. Using the result earlier that $\mathcal{P}=2 A$ the $\operatorname{spin}^{c}$ equation (2.20) may be rewritten as

$$
\left(\hat{\nabla}_{\mu}-\mathrm{i} A_{\mu}\right) \zeta=0
$$

which may already be compared with the Killing spinor equation (2.3).

More concretely, the solution to (2.20), or equivalently (2.21), is simply given by a constant spinor $\zeta$, so that $\partial_{\mu} \zeta=0$. This equation makes sense globally as $\zeta$ may be identified with a complex-valued function. To see this it is useful to take the following projection conditions

$$
\hat{\Gamma}_{1} \zeta=\mathrm{i} \hat{\Gamma}_{0} \zeta, \quad \hat{\Gamma}_{3} \zeta=\mathrm{i} \hat{\Gamma}_{2} \zeta
$$

following e.g. reference [32]. Here $\hat{\Gamma}_{a}, a=0,1,2,3$, denote the gamma matrices in the orthonormal frame (2.10). ${ }^{4}$ The covariant derivative of $\zeta$ is then computed to be

$$
\hat{\nabla}_{\mu} \zeta=\left(\partial_{\mu}+\frac{1}{4} \hat{\omega}_{\mu}^{\nu \rho} \hat{\Gamma}_{\nu \rho}\right) \zeta=\partial_{\mu} \zeta+\frac{\mathrm{i}}{2}\left(\hat{\omega}_{\mu}^{01}+\hat{\omega}_{\mu}^{23}\right) \zeta=\partial_{\mu} \zeta+\mathrm{i} A_{\mu} \zeta
$$

where $\hat{\omega}_{\mu}^{\nu \rho}$ is the spin connection of the conformal Kähler metric, and we have used the explicit form of this in appendix A together with the formula (2.8) for $A$. It follows that simply taking $\zeta$ to be constant, $\partial_{\mu} \zeta=0$, solves (2.20). This is a general phenomenon on any Kähler manifold.

Using the canonical spinor $\zeta$ we may construct a spinor $\epsilon$ that is a solution to the Killing spinor equation (2.3). Specifically, we find

$$
\epsilon=\frac{1}{\sqrt{2 y}}\left(1+V^{-1 / 2} \hat{\Gamma}_{0}\right) \zeta
$$

To verify this one first notes that the spin connections of the Kähler metric and the self-dual Einstein metric are related by

$$
\hat{\nabla}_{\mu} \zeta=\nabla_{\mu} \zeta+\frac{1}{2} \hat{\Gamma}_{\mu}{ }^{\nu}\left(\partial_{\nu} \log y\right) \zeta
$$

where $\hat{\Gamma}_{\mu}=y \Gamma_{\mu}$ in a coordinate basis. The Killing spinor equation then takes the form

$$
\left[\partial_{\mu}+\frac{1}{4} \hat{\omega}_{\mu}^{\nu \rho} \hat{\Gamma}_{\nu \rho}-\frac{1}{2} \hat{\Gamma}_{\mu}^{\nu}\left(\partial_{\nu} \log y\right)-\mathrm{i} A_{\mu}+\frac{1}{2 y} \hat{\Gamma}_{\mu}+\frac{\mathrm{i}}{4} y F_{\nu \rho} \hat{\Gamma}^{\nu \rho} \hat{\Gamma}_{\mu}\right] \epsilon=0 .
$$

\footnotetext{
${ }^{4}$ Strictly speaking the hats are redundant, but we keep them as a reminder that in this section the orthonormal frame is for the Kähler metric.
} 
To verify this is solved by (2.24) one simply substitutes (2.24) directly into the left-handside of (2.26). Using the explicit expressions for the spin connection, the gauge field, the field strength, as well as the projection conditions on the canonical spinor $\zeta$ and (2.20), one sees that (2.26) indeed holds.

From this analysis we can conclude that the self-dual Einstein metric (2.4) and the gauge field (2.8), which are solutions to Einstein-Maxwell theory in four dimensions, yield a Dirac spinor $\epsilon$ that is solution to the Killing spinor equation (2.3). This implies that these self-dual Einstein backgrounds are always locally supersymmetric solutions of Euclidean $\mathcal{N}=2$ gauged supergravity. We turn to global issues in the next section.

\section{Asymptotically locally AdS solutions}

In this section and the next we will assume that we are given a complete (non-singular) selfdual Einstein metric with a Killing vector, which then necessarily takes the local form (2.4). Moreover, we shall assume this metric is asymptotically locally Euclidean AdS, ${ }^{5}$ and in later subsections also that the four-manifold $M_{4}$ on which the metric is defined is topologically a ball. A two-parameter family of such self-dual solutions on the four-ball, generalizing all previously known solutions of this type, was constructed in [10]. In section 5 we shall review these solutions, and also introduce a number of further generalizations. In particular, the results of the current section allow us to deform the choice of Killing vector (which was essentially fixed in previous results), and we will also explain how to generalize to an infinite-dimensional family of solutions satisfying the above properties, starting with the local metrics in [26].

With the above assumptions in place, we begin in this section by showing that if the Killing vector $K=\partial_{\psi}$ is nowhere zero in a neighbourhood of the conformal boundary threemanifold $M_{3}$ then it is a Reeb vector field for an almost contact structure on $M_{3}$. We then reproduce the same geometric structure on $M_{3}$ studied from a purely three-dimensional viewpoint in [25]. In particular the asymptotic expansion of the Killing spinor $\epsilon$ leads to the same Killing spinor equation as [25]. This is important, as it shows that the dual field theory is defined on a supersymmetric background of the form studied in [25], for which the exact partition function of a general $\mathcal{N}=2$ supersymmetric gauge theory was computed in [20] using localization. Having studied the conformal boundary geometry, we then turn to the bulk in section 3.4. In particular we show that, with an appropriate restriction on the Killing vector $K$, the conformal Kähler structure of section 2.2 is everywhere non-singular. This allows us to prove in turn that the instanton and Killing spinor defined by the Kähler structure are everywhere non-singular.

In particular this means that each of the self-dual Einstein metrics in section 5 leads to a one-parameter family (depending on the choice of Killing vector $K$ ) of smooth supersymmetric solutions. In other words, if the self-dual Einstein metric depends on $n$ parameters, the complete solution will depend on $n+1$ parameters. We emphasize that in the previ-

\footnotetext{
${ }^{5}$ Since the metric has Euclidean signature one might more accurately describe this boundary condition as asymptotically locally hyperbolic, which is often used in the mathematics literature.
} 
ously known solutions the only example of this phenomenon is the solution of [6]. There the Einstein metric was simply $\mathrm{AdS}_{4}$, which doesn't have any parameters.

\subsection{Conformal boundary at $y=0$}

We are interested in self-dual Einstein metrics of the form (2.4) which are asymptotically locally Euclidean AdS (hyperbolic), in order to apply to the gauge/gravity correspondence. From the assumptions described above there is a single asymptotic region where the metric approaches $\frac{\mathrm{d} r^{2}}{r^{2}}+r^{2} \mathrm{~d} s_{M_{3}}^{2}$ as $r \rightarrow \infty$, where $M_{3}$ is a smooth compact three-manifold. In fact the metrics (2.4) naturally have such a conformal boundary at $y=0$. More precisely, we impose boundary conditions such that $w(y, z, \bar{z})$ is analytic around $y=0$, so

$$
w(y, z, \bar{z})=w_{(0)}(z, \bar{z})+y w_{(1)}(z, \bar{z})+\frac{1}{2} y^{2} w_{(2)}(z, \bar{z})+\mathcal{O}\left(y^{3}\right) .
$$

It follows that

$$
V(y, z, \bar{z})=1-\frac{1}{2} y w_{(1)}(z, \bar{z})-\frac{1}{2} y^{2} w_{(2)}(z, \bar{z})+\mathcal{O}\left(y^{3}\right)
$$

and that the metric $(2.4)$ is

$$
\mathrm{d} s_{\mathrm{SDE}}^{2}=[1+\mathcal{O}(y)] \frac{\mathrm{d} y^{2}}{y^{2}}+\frac{1}{y^{2}}\left[\left(\mathrm{~d} \psi+\phi_{0}\right)^{2}+4 \mathrm{e}^{w_{(0)}} \mathrm{d} z \mathrm{~d} \bar{z}+\mathcal{O}(y)\right]
$$

Setting $r=1 / y$ this is to leading order

$$
\mathrm{d} s_{\mathrm{SDE}}^{2} \simeq \frac{\mathrm{d} r^{2}}{r^{2}}+r^{2}\left[\left(\mathrm{~d} \psi+\phi_{0}\right)^{2}+4 \mathrm{e}^{w_{(0)}} \mathrm{d} z \mathrm{~d} \bar{z}\right]
$$

as $r \rightarrow \infty$, so that the metric is indeed asymptotically locally Euclidean AdS around $y=0$. Here we have also expanded the one-form tangent to $M_{3}$

$$
\left.\phi(y, z, \bar{z})\right|_{M_{3}}=\phi_{(0)}(z, \bar{z})+y \phi_{(1)}(z, \bar{z})+\mathcal{O}\left(y^{2}\right) .
$$

In fact by expanding (2.6) one can show that $\phi_{(1)}=0$. Of course, as usual one is free to redefine $r \rightarrow r \Omega(\psi, z, \bar{z})$, where $\Omega$ is any smooth, nowhere zero function on $M_{3}$, resulting in a conformal transformation of the boundary metric $\mathrm{d} s_{M_{3}}^{2} \rightarrow \Omega^{2} \mathrm{~d} s_{M_{3}}^{2}$. However, in the present context notice that $r=1 / y$ is a natural choice of radial coordinate.

With the analytic boundary condition (3.1) for $w$ it follows automatically that $K=\partial_{\psi}$ is nowhere zero in a neighbourhood of the conformal boundary $y=0$. As we shall see, this will reproduce the same structure on $M_{3}$ as [25], but we should stress that this is not the general situation. For example, one could take the standard hyperbolic metric for Euclidean AdS, conformally embedded as a unit ball in $\mathbb{R}^{4}$, and take $K$ to be the Killing vector that rotates the first factor in $\mathbb{R}^{2} \oplus \mathbb{R}^{2} \cong \mathbb{R}^{4}$. In fact this will be the natural choice of $K$ that arises in the two-monopole solution described in appendix C.3. The ansatz (3.1) is thus certainly a restriction on the class of possible globally regular solutions, although all examples in section 5 have choices of Killing vector for which this expansion holds. 
Returning to the case at hand, the conformal boundary is a compact three-manifold $M_{3}$ (by assumption), and from the above discussion a natural choice of representative for the metric is

$$
\mathrm{d} s_{M_{3}}^{2}=\left(\mathrm{d} \psi+\phi_{0}\right)^{2}+4 \mathrm{e}^{w_{(0)}} \mathrm{d} z \mathrm{~d} \bar{z}
$$

Notice that the form of the metric (3.6) is precisely of the form studied in [20]. In that reference an important role is played by the one-form

$$
\eta \equiv \mathrm{d} \psi+\phi_{0}
$$

which has exterior derivative

$$
\mathrm{d} \eta=\mathrm{d} \phi_{0}=\left.2 \mathrm{i} \partial_{y}\left(V \mathrm{e}^{w}\right)\right|_{y=0} \mathrm{~d} z \wedge \mathrm{d} \bar{z}=\mathrm{i} w_{(1)} \mathrm{e}^{w_{(0)}} \mathrm{d} z \wedge \mathrm{d} \bar{z} .
$$

The form $\eta$ is a global almost contact one-form on $M_{3}$. The most straightforward way to derive this in the case at hand is to note the form of the boundary Killing spinor equation in section 3.2 and appeal to the results of [25].

The Killing vector $K=\partial_{\psi}$ is the Reeb vector for the almost contact form $\eta$, as follows from the equations

$$
K\lrcorner \eta=1, \quad K\lrcorner \mathrm{d} \eta=0 .
$$

The orbits of $K$ thus foliate $M_{3}$, and moreover this foliation is transversely holomorphic with local complex coordinate $z$. When the orbits of $K$ all close it generates a U(1) symmetry of the boundary structure, and the orbit space $M_{3} / \mathrm{U}(1)$ is in general a compact orbifold surface, on which $z$ may be regarded as a local complex coordinate. These are generally called Seifert fibred three-manifolds in the literature. On the other hand, if $K$ has at least one non-closed orbit then since the isometry group of a compact manifold is compact, we deduce that $M_{3}$ admits at least a $\mathrm{U}(1) \times \mathrm{U}(1)$ symmetry, and the structure defined by $\eta$ is a toric almost contact structure. In this case we may introduce standard $2 \pi$-period coordinates $\varphi_{1}, \varphi_{2}$ on the torus $\mathrm{U}(1) \times \mathrm{U}(1)$ and write

$$
K=\partial_{\psi}=b_{1} \partial_{\varphi_{1}}+b_{2} \partial_{\varphi_{2}} .
$$

From (3.8) we deduce that the Taylor coefficient $w_{(1)}$ is a globally defined basic function on $M_{3}$ - that is, it is invariant under $K=\partial_{\psi}$. Moreover, the almost contact form $\eta$ is a contact form precisely when the function $w_{(1)}$ is everywhere positive. We shall see later that there are examples for which $\eta$ is contact and not contact. On the other hand, the coefficient $w_{(0)}$ is in general only a locally defined function of $z, \bar{z}$, as one sees by noting that the transverse metric $g_{T}=\mathrm{e}^{w_{(0)}} \mathrm{d} z \mathrm{~d} \bar{z}$ is a global two-tensor, but in general the complex coordinate $z$ is defined only locally. ${ }^{6}$ It will be useful in what follows to define a corresponding transverse volume form

$$
\operatorname{vol}_{T} \equiv 2 \mathrm{ie}^{w_{(0)}} \mathrm{d} z \wedge \mathrm{d} \bar{z}
$$

Again, this is a global tensor on $M_{3}$, with

$$
\mathrm{d} \eta=\mathrm{d} \phi_{0}=\frac{w_{(1)}}{2} \operatorname{vol}_{T} .
$$

\footnotetext{
${ }^{6}$ For example, for Euclidean $\mathrm{AdS}_{4}$ realized as a hyperbolic ball and with $K=\partial_{\psi}$ generating the Hopf fibration of the boundary $S^{3}$ then $g_{T}$ is the standard metric on the round two-sphere, implying that $w_{(0)}(z, \bar{z})=-2 \log \left(1+|z|^{2}\right)$ which blows up at $z=\infty$ (which is a smooth copy of $\left.S^{1} \subset M_{3} \cong S^{3}\right)$.
} 


\subsection{Boundary Killing spinor}

In this section we show that the Killing spinor $\epsilon$ induces a Killing spinor $\chi$ on the conformal boundary $M_{3}$ that solves the Killing spinor equation in [25].

We begin by recalling the orthonormal frame of one-forms

$$
e^{0}=\frac{1}{y} V^{1 / 2} \mathrm{~d} y, \quad e^{1}=\frac{1}{y} V^{-1 / 2}(\mathrm{~d} \psi+\phi), \quad e^{2}+\mathrm{i} e^{3}=\frac{2}{y}\left(V \mathrm{e}^{w}\right)^{1 / 2} \mathrm{~d} z,
$$

for the self-dual Einstein metric (2.4). We introduce a corresponding frame for the threemetric $\mathrm{d} s_{M_{3}}^{2}$ on the conformal boundary:

$$
e_{(3)}^{1}=\mathrm{d} \psi+\phi_{(0)}, \quad e_{(3)}^{2}+\mathrm{i} e_{(3)}^{3}=2 \mathrm{e}^{w_{(0)} / 2} \mathrm{~d} z,
$$

and will use indices $i, j, k=1,2,3$ for this orthonormal frame.

We next expand the four-dimensional Killing spinor equation (2.3) as a Taylor series in $y$. One starts by noting that $\Gamma^{\mu}=e^{\mu}{ }_{a} \Gamma^{a}=\mathcal{O}(y)$. But as $\Gamma_{\mu}=e^{a}{ }_{\mu} \Gamma_{a}=\mathcal{O}(1 / y)$ and the field strength expands as $F=F_{(0)}+y F_{(1)}+\mathcal{O}\left(y^{2}\right)$ we see that

$$
\frac{\mathrm{i}}{4} F_{\nu \rho} \Gamma^{\nu \rho} \Gamma_{\mu}=\mathcal{O}(y)
$$

After a computation we then obtain

$$
\left[\nabla_{\mu}^{(3)}-\mathrm{i} A_{(0) \mu}+\frac{1}{2 y}\left(1+\frac{1}{4} y w_{(1)}\right) e_{(3) \mu}^{i}\left(\Gamma_{i}-\Gamma_{i 0}\right)+\mathcal{O}(y)\right] \epsilon=0,
$$

where $\mu=\psi, z, \bar{z}$, and where

$$
A_{(0)}=-\frac{1}{4} w_{(1)} e_{(3)}^{1}+\frac{\mathrm{i}}{8} \mathrm{e}^{-w_{(0)} / 2}\left(\partial_{z}-\partial_{\bar{z}}\right) w_{(0)} e_{(3)}^{2}-\frac{1}{8} \mathrm{e}^{-w_{(0)} / 2}\left(\partial_{z}+\partial_{\bar{z}}\right) w_{(0)} e_{(3)}^{3},
$$

is the lowest order term in the expansion of $A$ given by (2.8). The Killing spinor $\epsilon$ then expands as

$$
\epsilon=\frac{1}{\sqrt{2 y}}\left[1+\Gamma_{0}+\frac{1}{4} y w_{(1)} \Gamma_{0}+\mathcal{O}\left(y^{2}\right)\right] \zeta_{0},
$$

where $\zeta_{0}$ is the lowest order ( $y$-independent) part of the Kähler spinor $\zeta$. Substituting this into (3.16) gives a leading order term that is identically zero. The subleading term then reads

$$
\left[\left(\nabla_{i}^{(3)}-\mathrm{i} A_{(0) i}\right)\left(1+\Gamma_{0}\right)+\frac{1}{8} w_{(1)}\left(\Gamma_{i 0}-\Gamma_{i}\right)\right] \zeta_{0}=0 .
$$

The projections (2.22), in the current context, read

$$
\Gamma_{1} \zeta_{0}=\mathrm{i} \Gamma_{0} \zeta_{0}, \quad \Gamma_{3} \zeta_{0}=\mathrm{i} \Gamma_{2} \zeta_{0} .
$$

We may choose the following representation of the gamma matrices:

$$
\Gamma_{i}=\left(\begin{array}{cc}
0 & \sigma_{i} \\
\sigma_{i} & 0
\end{array}\right), \quad \Gamma_{0}=\left(\begin{array}{cc}
0 & \mathrm{i} \mathbb{I}_{2} \\
-\mathrm{i} \mathbb{I}_{2} & 0
\end{array}\right),
$$


with $\sigma_{i}$ the Pauli matrices. ${ }^{7}$ The projection conditions then force $\zeta_{0}$ to take the form ${ }^{8}$

$$
\zeta_{0}=\left(\begin{array}{l}
\chi \\
0
\end{array}\right) \quad \text { where } \quad \chi=\left(\begin{array}{l}
\chi_{0} \\
\chi_{0}
\end{array}\right)
$$

Here $\chi$ is a two-component spinor and $\chi_{0}$ is simply a constant. The three-dimensional Killing spinor equation then becomes

$$
\left(\nabla_{i}^{(3)}-\mathrm{i} A_{(0) i}-\frac{\mathrm{i}}{8} w_{(1)} \sigma_{i}\right) \chi=0 .
$$

This three-dimensional Killing spinor equation is precisely of the form found in [25], and studied in [20]. More precisely, this is the form of the Killing spinor equation in the case where the background geometry has real-valued fields, with the metric given by (3.6), and the Killing spinor $\chi$ and its charge conjugate $\chi^{c}$ give rise to a supersymmetric background admitting two supercharges of opposite R-charge. In the notation of these references we have that the three-dimensional gauge field $V=0$ (or rather there exists a gauge in which this is true - see appendix B), while $A=A_{(0)}$ and the function $H=-\frac{\mathrm{i}}{4} w_{(1)}$. This result shows that there indeed exists a spinor $\chi$ with the required properties to construct supersymmetric field theories on $M_{3}$.

We close this subsection by remarking that supersymmetry singled out a natural representative (3.6) of the conformal class of the boundary metric. However, one is free to make the change in radial coordinate $r \rightarrow r \Omega$, with $\Omega$ any smooth, nowhere zero function on $M_{3}$, resulting in a conformal transformation of (3.6) by $\mathrm{d} s_{M_{3}}^{2} \rightarrow \Omega^{2} \mathrm{~d} s_{M_{3}}^{2}$. In particular, in the metric (3.6) the Killing vector $K=\partial_{\psi}$ has length 1 , while the latter conformal rescaling gives $\|K\|_{M_{3}}=\Omega$. In this case one instead finds that the vector $V$ in $[20,25]$ is non-zero, with gauge-invariant and generically non-zero components $V_{2}=\partial_{3} \log \Omega$ and $V_{3}=-\partial_{2} \log \Omega$. This is then in agreement with the three-dimensional results of [20]. For further details of this conformal rescaling we refer the reader to appendix B.

\subsection{Non-singular gauge}

In a neighbourhood of the conformal boundary the Kähler metric is defined on $[0, \epsilon) \times M_{3}$, for some $\epsilon>0$. This follows since via the conformal rescaling (2.9) the Kähler metric asymptotes to

$$
\mathrm{d} s_{\text {Kahler }}^{2} \simeq \mathrm{d} y^{2}+\mathrm{d} s_{M_{3}}^{2},
$$

near to the conformal boundary $y=0$. In particular the Kähler structure is smooth and globally defined in a neighbourhood of this boundary. Recall also that the gauge field $A$ is a connection on $\mathcal{K}^{-1 / 2}$. Since every orientable three-manifold is spin the canonical bundle

\footnotetext{
${ }^{7}$ In this basis the charge conjugation matrix $B$, appearing in $\epsilon^{c} \equiv B \epsilon^{*}$, is $B=\left(\begin{array}{cc}\varepsilon & 0 \\ 0 & -\varepsilon\end{array}\right)$ where $\varepsilon=$ $\left(\begin{array}{cc}0 & -1 \\ 1 & 0\end{array}\right)$

${ }^{8}$ Notice that although our frame coincides with that of [25], our three-dimensional gamma matrices are a permutation of those in the latter reference, which is why the spinor solution takes a slightly different form.
} 
$\mathcal{K}$ admits a square root in this neighbourhood, and so $A$ restricts to a bona fide connection one-form on $M_{3}$. The corresponding U(1) principal bundle can certainly be non-trivial for generic topology of $M_{3}$. In this section we analyse the simpler case where $M_{3} \cong S^{3}$. Here $A$ necessarily restricts to a global one-form $A_{(0)}$ on the conformal boundary, but as we shall see the explicit representative (3.17) is in a singular gauge. Correspondingly, since the boundary Killing spinor $\chi$ is a $\operatorname{spin}^{c}$ spinor, the solution (3.22) to (3.23) is similarly in a singular gauge. In this section we correct this by writing $A_{(0)}$ as a global one-form on $M_{3} \cong S^{3}$.

The expression (3.17) for the restriction of $A$ to the conformal boundary is of course only well-defined up to gauge transformations. We may rewrite the expression in (3.17) as

$$
A_{(0)}^{\text {local }}=-\frac{1}{4} w_{(1)}\left(\mathrm{d} \psi+\phi_{0}\right)+\frac{\mathrm{i}}{4} \partial_{z} w_{(0)} \mathrm{d} z-\frac{\mathrm{i}}{4} \partial_{\bar{z}} w_{(0)} \mathrm{d} \bar{z},
$$

adding the superscript label "local" to emphasize that in general this is only a local oneform. The first term is $-\frac{1}{4} w_{(1)} \eta$, which is always a global one-form on $M_{3}$, independently of the topology of $M_{3}$. However, the last two terms are not globally defined in general. We may remedy this in the case where $M_{3} \cong S^{3}$ by making a gauge transformation, adding an appropriate multiple of $\mathrm{d} \psi$ :

$$
A_{(0)}=-\frac{1}{4} w_{(1)} \eta+\gamma\left[\mathrm{d} \psi+\frac{\mathrm{i}}{4 \gamma} \partial_{z} w_{(0)} \mathrm{d} z-\frac{\mathrm{i}}{4 \gamma} \partial_{\bar{z}} w_{(0)} \mathrm{d} \bar{z}\right]
$$

This is then a global one-form on $M_{3} \cong S^{3}$ if and only if the curvature two-form of the connection in square brackets lies in the same basic cohomology class as $\mathrm{d} \eta=\mathrm{d} \phi_{0}$. Concretely, we write

$$
\gamma \mathrm{d} \psi+\frac{\mathrm{i}}{4} \partial_{z} w_{(0)} \mathrm{d} z-\frac{\mathrm{i}}{4} \partial_{\bar{z}} w_{(0)} \mathrm{d} \bar{z} \equiv \gamma \mathrm{d} \psi+B \equiv \gamma \eta+\alpha
$$

and compute

$$
\begin{aligned}
\mathrm{d} B & =-\frac{\mathrm{i}}{2} \partial_{z} \partial_{\bar{z}} w_{(0)} \mathrm{d} z \wedge \mathrm{d} \bar{z}=\left(w_{(1)}^{2}+w_{(2)}\right) \mathrm{e}^{w_{(0)}} \frac{\mathrm{i}}{2} \mathrm{~d} z \wedge \mathrm{d} \bar{z} \\
& =\frac{1}{4}\left(w_{(1)}^{2}+w_{(2)}\right) \operatorname{vol}_{T}
\end{aligned}
$$

where we used the Toda equation (2.7) and Taylor expanded. Since $\eta$ is a global one-form on $M_{3} \cong S^{3}$, it follows that (3.26) is a global one-form precisely if $\alpha$ defined via (3.27) is a global basic one-form, i.e. $\alpha$ is invariant under $\mathcal{L}_{\partial_{\psi}}$ and satisfies $\left.\partial_{\psi}\right\lrcorner \alpha=0$. In this case we have

$$
\int_{M_{3}} \eta \wedge \frac{1}{\gamma} \mathrm{d} B=\int_{M_{3}} \eta \wedge \mathrm{d} \eta
$$

which may be interpreted as saying that $\left[\frac{1}{\gamma} \mathrm{d} B\right]=[\mathrm{d} \eta] \in H_{\text {basic }}^{2}\left(M_{3}\right) \cong \mathbb{R}$ lie in the same basic cohomology class. Indeed, this is the case if and only if $\frac{1}{\gamma} \mathrm{d} B$ and $\mathrm{d} \eta$ differ by the exterior derivative of a global basic one-form.

The integral on the right hand side of (3.29) is the almost contact volume of $M_{3}$ :

$$
\mathrm{Vol}_{\eta} \equiv \int_{M_{3}} \eta \wedge \mathrm{d} \eta=\int_{M_{3}} \frac{w_{(1)}}{2} \eta \wedge \operatorname{vol}_{T}=\int_{M_{3}} \frac{w_{(1)}}{2} \sqrt{\operatorname{det} g_{M_{3}}} \mathrm{~d}^{3} x
$$


This played an important role in computing the classical localized Chern-Simons action in [20], which contributes to the field theory partition function on $M_{3}$. Using (3.28), (3.29) and (3.30) we see that $A_{(0)}$ in (3.26) is a global one-form if we choose the constant $\gamma$ via

$$
\frac{1}{4 \gamma} \int_{M_{3}}\left(w_{(1)}^{2}+w_{(2)}\right) \sqrt{\operatorname{det} g_{M_{3}}} \mathrm{~d}^{3} x=\mathrm{Vol}_{\eta} .
$$

We shall return to this formula in section 3.5

\subsection{Global conformal Kähler structure}

Recall that at the beginning of this section we assumed we were given a complete self-dual Einstein metric with Killing vector $K=\partial_{\psi}$, of the local form (2.4). We would like to understand when the conformal Kähler structure, studied locally in section 2.2, is then globally non-singular. As we shall see, this is not automatically the case. Focusing on the case of toric metrics on a four-ball (all examples in section 5 are of this type), with an appropriate restriction on $K$ we will see that the conformal Kähler structure is indeed everywhere regular. It follows in this case that the Kähler $\operatorname{spin}^{c}$ spinor and instanton $F=\frac{1}{2} \mathcal{R}$ are globally non-singular, and thus that the Killing spinor $\epsilon$ given by (2.24) is also globally defined and non-singular. Before embarking on this section, we warn the reader that the discussion is a little involved, and this section is probably better read in conjuction with the explicit examples in section 5. In fact the Euclidean $\mathrm{AdS}_{4}$ metric in section 5.1 displays almost all of the generic features we shall encounter.

The self-dual Einstein metrics of section 5 are all toric, and we may thus parameterize a choice of toric Killing vector $K$ as

$$
K=b_{1} \partial_{\varphi_{1}}+b_{2} \partial_{\varphi_{2}}
$$

where we have introduced standard $2 \pi$-period coordinates $\varphi_{1}, \varphi_{2}$ on the torus $\mathrm{U}(1) \times \mathrm{U}(1)$. It will be important to fix carefully the orientations here. Since the metrics are defined on a ball, diffeomorphic to $\mathbb{R}^{4} \cong \mathbb{R}^{2} \oplus \mathbb{R}^{2}$ with $\mathrm{U}(1) \times \mathrm{U}(1)$ acting in the obvious way, we choose $\partial_{\varphi_{i}}$ so that the orientations on $\mathbb{R}^{2}$ induce the given orientation on $\mathbb{R}^{4}$ (with respect to which the metric has anti-self-dual Weyl tensor). This fixes the relative sign of $b_{1}$ and $b_{2}$. Given that we have also assumed that $K$ has no fixed points near the conformal boundary, we must also have $b_{1}$ and $b_{2}$ non-zero. Thus $b_{1} / b_{2} \in \mathbb{R} \backslash\{0\}$, and its sign will be important in what follows.

Since the self-dual Einstein metric is assumed regular, the one-form $K^{b}$ and its exterior derivative $\mathrm{d} K^{b}$ are both globally defined and regular. The self-dual two-form

$$
\Psi \equiv\left(\mathrm{d} K^{b}\right)^{+} \equiv \frac{1}{2}\left(\mathrm{~d} K^{b}+* \mathrm{~d} K^{b}\right)
$$

is a twistor [26], and the invariant definition of the function/coordinate $y$ in section 2 is given in terms of its norm by

$$
\frac{2}{y^{2}}=\|\Psi\|^{2} \equiv \frac{1}{2 !} \Psi_{\mu \nu} \Psi^{\mu \nu}
$$


The complex structure tensor for the conformal Kähler structure is correspondingly

$$
J_{\nu}^{\mu}=-y \Psi_{\nu}^{\mu}
$$

where indices are raised and lowered using the self-dual Einstein metric. It is then an algebraic fact that $J^{2}=-1$. The conformal Kähler structure will thus be everywhere regular, provided the functions $y$ and $1 / y$ are not zero. Of course $y=0$ is the conformal boundary (which is at infinity, and is not part of the self-dual Einstein space). We are free to choose the sign when taking a square root of (3.34), and without loss of generality we take $y>0$ in a neighbourhood of the conformal boundary at $y=0$. Since everything is regular, in particular the norm of the twistor $\Psi$ cannot diverge anywhere (except at infinity), and thus $y \neq 0$ in the interior of the bulk $M_{4}$. It follows that $y$ is everywhere positive on $M_{4}$.

The Killing vector $K$ is zero only at the "NUT", namely the fixed origin of $\mathbb{R}^{4} \cong$ $\mathbb{R}^{2} \oplus \mathbb{R}^{2}$. At this point the two-form $\mathrm{d} K^{b}$, in an orthonormal frame, is a skew-symmetric $4 \times 4$ matrix whose weights are precisely the coefficients $b_{1}, b_{2}$ in $(3.32) .{ }^{9}$ It follows from the definitions (3.33) and (3.34), together with a little linear algebra in such an orthonormal frame, that

$$
y_{\mathrm{NUT}}=\frac{1}{\left|b_{1}+b_{2}\right|} .
$$

The conformal Kähler structure will thus be regular everywhere, except potentially where $1 / y=0$. Suppose that $1 / y=0$ at a point $p \in M_{4} \backslash\{\mathrm{NUT}\}$. Then $K=\left.\partial_{\psi}\right|_{p} \neq 0$, and thus from the metric (2.4) we see that $1 /\left.\left(V y^{2}\right)\right|_{p} \neq 0$. It follows that the function $V$ must tend to zero as $1 / y^{2}$ as one approaches $p$. We may thus write $V=\frac{c}{y^{2}}+o\left(1 / y^{2}\right)$, where $c=c(z, \bar{z})$ is non-zero at $p$. Using the definition of $V$ in terms of $w$ in (2.5) we thus see that $\partial_{y} w=\frac{2}{y}-\frac{2 c}{y^{3}}+o\left(1 / y^{3}\right)$. There are then various ways to see that the corresponding supersymmetric supergravity solution is singular. Perhaps the easiest is to note from the Killing spinor formula (2.24), together with the fact that we may normalise $\zeta^{\dagger} \zeta=1$, we have

$$
\epsilon^{\dagger} \epsilon=\frac{1}{2 y}\left(1+V^{-1}\right)
$$

which from the above behaviour of $V$ then diverges as we approach the point $p$. It follows that the Killing spinor $\epsilon$ is divergent at $p$, and the solution is singular.

The solutions are thus singular on $M_{4} \backslash\{\mathrm{NUT}\}$ if and only if $\{1 / y=0\} \backslash\{\mathrm{NUT}\}$ is non-empty. Since $y_{\mathrm{NUT}}=1 /\left|b_{1}+b_{2}\right|$, the analysis will be a little different for the cases $b_{1} / b_{2}=-1$ and $b_{1} / b_{2} \neq-1$. We thus assume the latter (generic) case for the time being. As in the last paragraph, let us suppose $1 /\left.y\right|_{p}=0$. Due to the behaviour of $V$ and $w$ near $p$, it follows from the form of the metric (2.4) that $p$ must lie on one of the axes, i.e. at $\rho_{1}=0$ or at $\rho_{2}=0$, where $\left(\rho_{i}, \varphi_{i}\right)$ are standard polar coordinates on each copy of $\mathbb{R}^{2} \oplus \mathbb{R}^{2} \cong \mathbb{R}^{4} \cong M_{4}, i=1,2 .{ }^{10}$ In either case there is then an $S^{1} \ni p$ locus of points where $1 / y=0$, as follows by following the orbits of the Killing vector $\partial_{\varphi_{2}}$ or $\partial_{\varphi_{1}}$, respectively.

\footnotetext{
${ }^{9}$ This is perhaps easiest to see by noting that to leading order the metric is flat at the NUT, so one can compute $\mathrm{d} K^{\mathrm{b}}$ in an orthonormal frame at the NUT using the flat Euclidean metric on $\mathbb{R}^{2} \oplus \mathbb{R}^{2}$.

${ }^{10}$ Notice that when $b_{1} / b_{2}=-1$ in fact $1 / y=0$ at the NUT itself, $\rho_{1}=\rho_{2}=0$.
} 
To see when this happens, our analysis will be based on the fact that, since the Killing vector has finite norm in the interior of $M_{4}$, one can straightforwardly show that $y$ diverges if and only if $\|\mathrm{d} y\|=0$. It is then convenient to consider the function $y$ restricted to the relevant axis, i.e. $\left.y\right|_{\left\{\rho_{1}=0\right\}} \equiv y_{2}\left(\rho_{2}\right)$ or $\left.y\right|_{\left\{\rho_{2}=0\right\}} \equiv y_{1}\left(\rho_{1}\right)$. We have $y_{1}(0)=y_{2}(0)=y_{\text {NUT }}>$ 0 . Suppose that $y_{i}(\rho)$ (for either $\left.i=1,2\right)$ starts out decreasing along the axis as we move away from the NUT. Then in fact it must remain monotonic decreasing along the whole axis, until it reaches $y=0$ at conformal infinity where $\rho=\infty$. The reason for this is simply that if $y_{i}(\rho)$ has a turning point then ${ }^{11} \mathrm{~d} y=0$, which we have already seen can happen only where $y$ diverges: but this contradicts the fact that $y_{i}(\rho)$ is decreasing from a positive value at $\rho=0$ (and is bounded below by 0 ). On the other hand, suppose that $y_{i}(\rho)$ starts out increasing at the NUT. Then since at conformal infinity $y_{i}(\infty)=0$, it follows that $y_{i}(\rho)$ must have a turning point at some finite $\rho>0$. At such a point $y$ will diverge, and from our above discussion the solution is singular.

This shows that the key is to examine $\mathrm{d} y$ at the NUT itself. Recall that the coordinate $y$ is a Hamiltonian function for the Killing vector $K$, i.e. $\mathrm{d} y=-K\lrcorner \omega$. From (3.35), we also know that $\omega$ is related to the two-form $\Psi=\left(\mathrm{d} K^{b}\right)^{+}$by $\omega=-y^{3} \Psi$, yielding $\left.\mathrm{d} y=y^{3} K\right\lrcorner\left(\mathrm{d} K^{b}\right)^{+}$. At the NUT we may again use the polar coordinates $\left(\rho_{i}, \varphi_{i}\right)$ for the two copies of $\mathbb{R}^{2}$, where the metric is to leading order the metric on flat space. In the usual orthonormal frame for these polar coordinates, using the above formulae we then compute to leading order

$$
\left.(\mathrm{d} y)\right|_{\mathrm{NUT}} \simeq\left(\begin{array}{cc}
-\frac{b_{1}}{\left(b_{1}+b_{2}\right)^{2}} & \operatorname{sign}\left(b_{1}+b_{2}\right) \rho_{1} \\
0 \\
-\frac{b_{2}}{\left(b_{1}+b_{2}\right)^{2}} & \operatorname{sign}\left(b_{1}+b_{2}\right) \rho_{2} \\
0
\end{array}\right)
$$

Thus when $b_{1} / b_{2}>0$ we see that $y_{i}(\rho)$ starts out decreasing at the NUT, for both $i=1,2$, and from the previous paragraph it follows that the solution is then globally non-singular! On the other hand, the case $b_{1} / b_{2}<0$ splits further into two subcases. For simplicity let us describe the case where $b_{2}>0$ (with the case $b_{2}<0$ being similar). Then when $b_{1} / b_{2}<-1$ we have $y_{2}(\rho)$ starts out increasing at the NUT, which then leads to a singularity along the axis $\rho_{1}=0$ at some finite value of $\rho_{2}$; on the other hand, when $-1<b_{1} / b_{2}<0$ we have that $y_{1}(\rho)$ starts out increasing at the NUT, which then leads to a singularity along the axis $\rho_{2}=0$ at some finite value of $\rho_{1}$. Notice these two subcases meet where $b_{1} / b_{2}=-1$, when we know that $1 / y=0$ at the NUT itself, $\rho_{1}=\rho_{2}=0$.

This leads to the simple picture that all solutions with $b_{1} / b_{2}>0$ are globally regular, while all solutions with $b_{1} / b_{2}<0$ are singular, except when $b_{1} / b_{2}=-1$. In this latter case $y$ is infinity at the NUT. As one moves out along either axis $y$ is then necessarily monotonically decreasing to zero, by similar arguments to those above. Thus the $b_{1} / b_{2}=$ -1 solution is in fact also non-singular, although qualitatively different from the solutions with $b_{1} / b_{2}>0$. One can show that, regardless of the values of $b_{1}$ and $b_{2}$, the complex structure (3.35) is always the standard complex structure on flat space at the NUT, meaning

\footnotetext{
${ }^{11}$ Notice that $\mathrm{d} y$ necessarily points along the axis, given the form of the metric (2.4).
} 
that when $b_{1} / b_{2}>0$ the induced complex structure at the NUT is $\mathbb{C}^{2}$, while when $b_{1} / b_{2}=$ -1 the NUT becomes a point at infinity in the conformal Kähler metric, with the Kähler metric being asymptotically Euclidean. In particular the instanton is zero at the NUT in this case, and so is regular there.

Notice that, for the regular solutions, since $K$ is nowhere zero away from the NUT we may deduce that also $\mathrm{d} y=-K\lrcorner \omega$ is nowhere zero (as $\omega$ is a global symplectic form on $\left.M_{4} \backslash\{\mathrm{NUT}\}\right)$. In particular $y$ is a global Hamiltonian function for $K$, and in particular it is a Morse-Bott function on $M_{4}$. This implies that $y$ has no critical points on $M_{4} \backslash\{\mathrm{NUT}\}$, and thus that $y_{\mathrm{NUT}}$ is the maximum value of $y$ on $M_{4}$. Moreover, the Morse-Bott theory tells us that constant $y$ surfaces on $M_{4} \backslash\{\mathrm{NUT}\}$ are all diffeomorphic to $M_{3} \cong S^{3}$.

We shall see all of the above behaviour very explicitly in section 5 for the case when the self-dual Einstein metric is simply Euclidean $\mathrm{AdS}_{4}$. The more complicated Einstein metrics in that section of course also display these features, although the corresponding formulae become more difficult to make completely explicit as the examples become more complicated.

\subsection{Toric formulae}

In this section we shall obtain some further formulae, valid for any toric self-dual Einstein metric on the four-ball. These will be useful for computing the holographic free energy in the next section.

We first note that for $M_{3} \cong S^{3}$ with Reeb vector (3.10) the almost contact volume in (3.30) may be computed using equivaraint localization to give

$$
\mathrm{Vol}_{\eta}=\int_{M_{3}} \eta \wedge \mathrm{d} \eta=-\frac{(2 \pi)^{2}}{b_{1} b_{2}} .
$$

This formula also appeared in [20], although in the present paper we have been more careful with sign conventions. One proves (3.39) by an analogous computation to the Duistermaat-Heckman formula in [33]. Specifically, we define a two-form

$$
\tilde{\omega} \equiv \frac{1}{2} \mathrm{~d}\left(\varrho^{2} \eta\right)
$$

on $M_{4}$, where $\varrho$ is a choice of radial coordinate with the NUT at $\varrho=0$ and the conformal boundary at $\varrho=\infty$, and notice that

$$
\mathrm{Vol}_{\eta}=-\int_{M_{4}} \mathrm{e}^{-\varrho^{2} / 2} \frac{1}{2 !} \tilde{\omega} \wedge \tilde{\omega} .
$$

The minus sign arises here because the natural orientation on $M_{3}$ defined in our set-up is opposite to that on the right hand side of (3.41). Specifically, $y$ is decreasing towards the boundary of $M_{4}$, so that dy points inwards from $M_{3}=\partial M_{4}$, while $\varrho$ is increasing towards the boundary, with d $\varrho$ pointing outwards. ${ }^{12}$ One then evaluates the right hand side of (3.41) using equivariant localization. Specifically, the integrand is

$$
\exp \left[-\frac{\varrho^{2}}{2}+\tilde{\omega}\right]
$$

\footnotetext{
${ }^{12}$ Notice that we could have avoided this by choosing $y$ to be strictly negative on the interior of $M_{4}$, rather than strictly positive.
} 
which since $K\lrcorner \tilde{\omega}=-\mathrm{d}\left(\frac{\varrho^{2}}{2}\right)$ is an equivariantly closed form for $K$, i.e. is closed under $\left.\mathrm{d}+K\right\lrcorner$. The Berline-Vergne equivariant integration theorem then localizes the integral to the fixed point set of $K$, and one obtains precisely (3.39), with the $b_{i}$ appearing as the weights of the action of $K$ at the NUT. ${ }^{13}$

Finally, let us return to the equation (3.31). In fact there is another interpretation of the constant $\gamma$, in terms of the charge of the Killing spinor under $K$. To see this, recall that the solution (3.22) to the three-dimensional Killing spinor equation (3.23) is simply constant in our frame, but that was for the case where the gauge field $A_{(0)}$ is given by (3.25), which as we saw in section 3.3 is always in a singular gauge on $M_{3} \cong S^{3}$. The gauge transformation $A_{(0)} \rightarrow A_{(0)}+\gamma \mathrm{d} \psi$ that we made in (3.26) to obtain a non-singular gauge implies that the correct global spinor $\chi$ has a phase dependence

$$
\chi^{\text {global }}=\mathrm{e}^{\mathrm{i} \gamma \psi}\left(\begin{array}{l}
\chi_{0} \\
\chi_{0}
\end{array}\right),
$$

where $\chi_{0}$ is a constant complex number. Since the frame is invariant under $K=\partial_{\psi}$, we thus deduce that $\gamma$ is precisely the charge of the Killing spinor under $K$.

On the other hand, the total four-dimensional spinor is constructed from the canonical spinor $\zeta$ on the conformal Kähler manifold, via (2.24). Thus $\gamma$ is also the charge of $\zeta$ under $K$. This immediately allows us to write down that

$$
|\gamma|=\frac{\left|b_{1}\right|+\left|b_{2}\right|}{2}
$$

This formula may be fixed by looking at the behaviour at the NUT, where recall that the complex structure is that of $\mathbb{C}^{2}$. In terms of complex coordinates $z_{1}=\left|z_{1}\right| \mathrm{e}^{\mathrm{i} \psi_{1}}, z_{2}=\left|z_{2}\right| \mathrm{e}^{\mathrm{i} \psi_{2}}$, the Kähler spinor $\zeta$, and hence also our Killing spinor, has charges $\frac{1}{2}$ under each of $\partial_{\psi_{i}}$, $i=1,2$. However, one must be careful to correctly fix the orientations, which leads to the modulus signs in (3.44). More precisely, for $b_{1} / b_{2}>0$ the conformal Kähler metric fills the interior of a ball in $\mathbb{C}^{2}$, while for $b_{1} / b_{2}=-1$ instead it is the exterior - see, for example, the discussion at the end of section 5.1.

\section{Holographic free energy}

In this section we compute the regularized holographic free energy for a supersymmetric self-dual asymptotically locally Euclidean AdS solution defined on the four-ball, deriving the remarkably simple formula (1.2) quoted in the introduction.

\subsection{General formulae}

The computation of the holographic free energy follows standard holographic renormalization methods $[34,35]$. The total on-shell action is

$$
I=I_{\mathrm{bulk}}^{\mathrm{grav}}+I^{F}+I_{\mathrm{bdry}}^{\mathrm{grav}}+I_{\mathrm{ct}}^{\text {grav }} .
$$

\footnotetext{
${ }^{13}$ This is then the Duistermaat-Heckman formula when $\tilde{\omega}$ is a symplectic form, i.e. when $\eta$ is a contact form.
} 
Here the first two terms are the bulk (Euclidean) supergravity action (2.1)

$$
I^{\mathrm{SUGRA}}=I_{\mathrm{bulk}}^{\mathrm{grav}}+I^{F} \equiv-\frac{1}{16 \pi G_{4}} \int_{M_{4}}\left(R+6-F^{2}\right) \sqrt{\operatorname{det} g} \mathrm{~d}^{4} x,
$$

evaluated on a particular solution with topology $M_{4}$. The boundary term $I_{\text {bdry }}^{\text {grav }}$ in (4.1) is the Gibbons-Hawking-York term, required so that the equations of motion (2.2) follow from the bulk action (4.2) for a manifold $M_{4}$ with boundary. This action is divergent, but we may regularize it using holographic renormalization. Introducing a cut-off at a sufficiently small value of $y=\delta>0$, with corresponding hypersurface $\mathcal{S}_{\delta}=\{y=\delta\} \cong M_{3}$, we have the following total boundary terms

$$
I_{\mathrm{bdry}}^{\text {grav }}+I_{\mathrm{ct}}^{\text {grav }}=\frac{1}{8 \pi G_{4}} \int_{\mathcal{S}_{\delta}}\left(-K+2+\frac{1}{2} R(h)\right) \sqrt{\operatorname{det} h} \mathrm{~d}^{3} x .
$$

Here $R(h)$ is the Ricci scalar of the induced metric $h_{i j}$ on $\mathcal{S}_{\delta}$, and $K$ is the trace of the second fundamental form of $\mathcal{S}_{\delta}$, the latter being the Gibbons-Hawking-York boundary term. It is convenient to rewrite the latter using

$$
\int_{\mathcal{S}_{\delta}} K \sqrt{\operatorname{det} h} \mathrm{~d}^{3} x=\mathcal{L}_{n} \int_{\mathcal{S}_{\delta}} \sqrt{\operatorname{det} h} \mathrm{~d}^{3} x
$$

where $n$ is the outward pointing normal vector to the boundary $\mathcal{S}_{\delta}$.

\subsection{The four-ball}

In this section we evaluate the total free energy (4.1) in the case of a supersymmetric self-dual solution on the four-ball $M_{4} \cong B^{4} \cong \mathbb{R}^{4}$.

We deal with each term in (4.1) in turn, beginning with the gauge field contribution

$$
I^{F}=\frac{1}{16 \pi G_{4}} \int_{M_{4}} F^{2} \sqrt{\operatorname{det} g} \mathrm{~d}^{4} x=-\frac{1}{8 \pi G_{4}} \int_{M_{4}} F \wedge F=\int_{M_{3}} A_{(0)} \wedge F_{(0)} .
$$

Here in second equality we have used the fact that $*_{4} F=-F$ is anti-self-dual, while in the last equality we used the fact that on the four-ball $M_{4}=B^{4} \cong \mathbb{R}^{4}$ the curvature $F=\mathrm{d} A$ is globally exact. Thus we may apply Stokes' theorem with $M_{3}=\partial M_{4}$, recalling that the natural orientation on $M_{3}$ is induced from an inward-pointing normal vector, as in the discussion of (3.41). ${ }^{14}$ Notice also that here the gauge field action is already finite, so there is no need to realize the conformal boundary $M_{3}$ as the $\operatorname{limit}_{\lim _{\delta \rightarrow 0}} \mathcal{S}_{\delta}$. Next we compute the integrand in (4.5) using the global form of $A_{(0)}$ (3.26) in section 3.3. Recall that this reads

$$
A_{(0)}=-\frac{1}{4} w_{(1)} \eta+\gamma \mathrm{d} \psi+B=-\frac{1}{4} w_{(1)} \eta+\gamma \eta+\alpha,
$$

where in particular $\alpha$ is a global basic one-form. We then compute

$$
\begin{aligned}
A_{(0)} \wedge F_{(0)}= & \frac{w_{(1)}^{3}}{32} \eta \wedge \operatorname{vol}_{T}-\frac{1}{4} w_{(1)} \eta \wedge \mathrm{d} B-\frac{\gamma}{8} w_{(1)}^{2} \eta \wedge \operatorname{vol}_{T} \\
& +\gamma \eta \wedge \mathrm{d} B-\frac{1}{4} \alpha \wedge \mathrm{d} w_{(1)} \wedge \eta .
\end{aligned}
$$

\footnotetext{
${ }^{14}$ Concretely, the integral over $y$ is $\int_{y_{\mathrm{NUT}}}^{0} \mathrm{~d} y$, where we chose the convention that $y_{\mathrm{NUT}}>0$.
} 
When we integrate this over $M_{3}$, the last term may be integrated by parts, giving an integral that is equal to the integral of $-\frac{1}{4} w_{(1)} \eta \wedge \mathrm{d} \alpha$, which then combines with the first line of (4.7). On the other hand, the first term on the second line of (4.7) may be evaluted in the $\mathrm{U}(1) \times$ $\mathrm{U}(1)$ toric case using (3.28), the integral (3.31) and the formula (3.44) for $|\gamma|$. This leads to

$$
\begin{aligned}
I^{F}= & -\frac{\pi}{2 G_{4}} \cdot \frac{\left(\left|b_{1}\right|+\left|b_{2}\right|\right)^{2}}{4 b_{1} b_{2}}+\frac{1}{8 \pi G_{4}} \int_{M_{3}} \frac{w_{(1)}^{3}}{32} \sqrt{\operatorname{det} g_{M_{3}}} \mathrm{~d}^{3} x \\
& -\frac{1}{8 \pi G_{4}} \int_{M_{3}} \frac{1}{8}\left(w_{(1)}^{3}+w_{(1)} w_{(2)}\right) \sqrt{\operatorname{det} g_{M_{3}}} \mathrm{~d}^{3} x .
\end{aligned}
$$

Notice that the first term closely resembles the free energy appearing in (1.2) - we shall see momentarily that this combines with a term coming from the gravitational contribution.

We turn next to the bulk gravity part of the action, which when evaluated on-shell is

$$
I_{\mathrm{bulk}}^{\mathrm{grav}}=\frac{1}{16 \pi G_{4}} \int_{M_{4}^{\delta}} 6 \mathrm{vol}_{4} .
$$

Here $M_{4}^{\delta}$ is cut off along the boundary $\mathcal{S}_{\delta}=\{y=\delta\} \cong M_{3}$, which is necessary as the volume is of course divergent. The volume form of interest is

$$
\operatorname{vol}_{4}=\frac{1}{y^{4}} \mathrm{~d} y \wedge(\mathrm{d} \psi+\phi) \wedge V \mathrm{e}^{w} 2 \mathrm{id} z \wedge \mathrm{d} \bar{z} .
$$

A computation reveals that this may be written as the exact form

$$
-3 \mathrm{vol}_{4}=\mathrm{d} \Gamma
$$

where we have defined the three-form

$$
\Gamma \equiv \frac{1}{2 y^{2}}(\mathrm{~d} \psi+\phi) \wedge \mathrm{d} \phi+\frac{1}{y^{3}}(\mathrm{~d} \psi+\phi) \wedge V \mathrm{e}^{w} 2 \mathrm{id} z \wedge \mathrm{d} \bar{z} .
$$

We may then integrate over $M_{4}^{\delta}$ using Stokes' theorem. To do this let us define $\varrho$ to be geodesic distance from the NUT - the origin of $M_{4} \cong B^{4} \cong \mathbb{R}^{4}$ that is fixed by the Killing vector $K=\partial_{\psi}$. We then more precisely cut off the space also at small $\varrho>0$ and let $\varrho \rightarrow 0$, so that we are integrating over $M_{4}^{\delta, \varrho}$. The form $\Gamma$ may be written

$$
\Gamma=\frac{1}{2 y^{2}}(\mathrm{~d} \psi+\phi) \wedge \mathrm{d} \phi+\frac{1}{y^{3}}(\mathrm{~d} \psi+\phi) \wedge \omega,
$$

where $\omega$ is the conformal Kähler form. As argued in section 3.4, when $y_{\mathrm{NUT}}$ is finite $\omega$ is everywhere a smooth two-form, and thus in particular in polar coordinates near the NUT at $\varrho=0$ it takes the form $\omega \simeq \varrho \mathrm{d} \varrho \wedge \beta_{1}+\varrho^{2} \beta_{2}$ to leading order, where $\beta_{1}$ and $\beta_{2}$ are pullbacks of smooth forms on the $S^{3}=S_{\mathrm{NUT}}^{3}$ at constant $\varrho>0$. Because of this, the second term in (4.13) does not contribute to the integral around the NUT. However, notice that

$$
\int_{S_{\mathrm{NUT}}^{3}}(\mathrm{~d} \psi+\phi) \wedge \mathrm{d} \phi=\int_{M_{3}^{y=0}}(\mathrm{~d} \psi+\phi) \wedge \mathrm{d} \phi=-\frac{(2 \pi)^{2}}{b_{1} b_{2}},
$$


follows from a simple application of Stokes' theorem, where we have used the almost contact volume (3.39). Using the fact (3.36) that $y_{\mathrm{NUT}}=1 /\left|b_{1}+b_{2}\right|$ one thus obtains

$$
\int_{M_{4}^{\delta}} \operatorname{vol}_{4}=\frac{(2 \pi)^{2}\left|b_{1}+b_{2}\right|^{2}}{6 b_{1} b_{2}}+\int_{M_{3}^{y=0}}\left[\frac{1}{3 \delta^{3}}+\frac{w_{(1)}}{4 \delta^{2}}\right] \sqrt{\operatorname{det} g_{M_{3}}} \mathrm{~d}^{3} x
$$

so that

$$
\begin{aligned}
I_{\text {bulk }}^{\text {grav }}= & \frac{\pi}{2 G_{4}} \cdot \frac{\left|b_{1}+b_{2}\right|^{2}}{2 b_{1} b_{2}}+\frac{1}{8 \pi G_{4}} \cdot \frac{1}{\delta^{3}} \int_{M_{3}^{y=0}} \sqrt{\operatorname{det} g_{M_{3}}} \mathrm{~d}^{3} x \\
& +\frac{3}{32 \pi G_{4}} \cdot \frac{1}{\delta^{2}} \int_{M_{3}^{y=0}} w_{(1)} \sqrt{\operatorname{det} g_{M_{3}}} \mathrm{~d}^{3} x .
\end{aligned}
$$

In particular notice that the $\mathcal{O}(0)$ term at the conformal boundary is zero. This follows from the identity

$$
\int_{M_{3}}\left(w_{(1)}^{3}+3 w_{(1)} w_{(2)}+w_{(3)}\right) \sqrt{\operatorname{det} g_{M_{3}}} \mathrm{~d}^{3} x=0,
$$

which arises from Taylor expanding the Toda equation (2.7) as

$$
\begin{aligned}
0= & \partial_{z} \partial_{\bar{z}} w_{(0)}+\mathrm{e}^{w_{(0)}}\left(w_{(1)}^{2}+w_{(2)}\right) \\
& +y\left[\partial_{z} \partial_{\bar{z}} w_{(1)}+\mathrm{e}^{w_{(0)}}\left(w_{(1)}^{3}+3 w_{(1)} w_{(2)}+w_{(3)}\right)\right]+\mathcal{O}\left(y^{2}\right) .
\end{aligned}
$$

In particular, because $w_{(1)}$ is a smooth global function on $M_{3}$, the second line implies (4.17).

It remains to evaluate the boundary terms $I_{\mathrm{bdry}}^{\text {grav }}+I_{\mathrm{ct}}^{\text {grav }}$. After a computation, and again using (4.17), one obtains

$$
\begin{aligned}
I_{\mathrm{bdry}}^{\mathrm{grav}}+I_{\mathrm{ct}}^{\mathrm{grav}}= & -\frac{1}{8 \pi G_{4} \delta^{3}} \int_{M_{3}^{y=0}} \sqrt{\operatorname{det} g_{M_{3}}} \mathrm{~d}^{3} x-\frac{3}{32 \pi G_{4} \delta^{2}} \int_{M_{3}^{y=0}} w_{(1)} \sqrt{\operatorname{det} g_{M_{3}}} \mathrm{~d}^{3} x \\
& +\frac{1}{256 \pi G_{4}} \int_{M_{3}}\left(3 w_{(1)}^{3}+4 w_{(1)} w_{(2)}\right) \sqrt{\operatorname{det} g_{M_{3}}} \mathrm{~d}^{3} x .
\end{aligned}
$$

Adding (4.19) to the bulk gravity term (4.16) we see that the divergent terms do indeed precisely cancel, and further combining with (4.8) we see that the terms involving the integrals of $w_{(i)}$ also all cancel.

The computations we have done are valid only for globally regular solutions, and recall these divide into the two cases $b_{1} / b_{2}>0$, and $b_{1} / b_{2}=-1$. In the first case the first term in (4.8) combines with the first term in (4.16) to give

$$
I=\frac{\pi}{2 G_{4}} \cdot \frac{\left(\left|b_{1}\right|+\left|b_{2}\right|\right)^{2}}{4\left|b_{1} b_{2}\right|},
$$

where notice $\left|b_{1}+b_{2}\right|=\left|b_{1}\right|+\left|b_{2}\right|$. On the other hand the isolated case with $b_{1} / b_{2}=-1$ has $b_{1}+b_{2}=0$, so that the free energy comes entirely from the first term in (4.8), which remarkably is then also given by the formula (4.20). Thus for all regular supersymmetric solutions we have shown that (4.20) holds. 


\subsection{Index theory formulae}

Although our main result (4.20) is extremely simple, it is also possible to derive another interesting formula for the holographic free energy (that however seems less practically useful). We begin by following [36], which rewrites the gravitational contribution

$$
I^{\text {grav }}=I_{\mathrm{bulk}}^{\text {grav }}+I_{\mathrm{bdry}}^{\text {grav }}+I_{\mathrm{ct}}^{\text {grav }}
$$

to the total holographic free energy $I=I^{\text {grav }}+I^{F}$. Specifically, we may use the GaussBonnet formula to rewrite $I^{\text {grav }}$ as [36]

$$
I^{\text {grav }}=\frac{\pi}{2 G_{4}} \chi\left(M_{4}\right)-\frac{1}{16 \pi G_{4}} \int_{M_{4}}|W|^{2} \sqrt{\operatorname{det} g} \mathrm{~d}^{4} x,
$$

where $W$ denotes the Weyl tensor and $\chi\left(M_{4}\right)$ is the Euler number of $M_{4}$. For example, for Euclidean AdS, which is conformally flat and has the topology of a four-ball, (4.22) immediately gives $I=\frac{\pi}{2 G_{4}}$.

When the metric on $M_{4}$ is also anti-self-dual one can go further, using the AtiyahPatodi-Singer index theorem [37]. This was first applied, in the current context, in [38]. The index theorem for the signature operator in general reads [37]

$$
\sigma\left(M_{4}\right)=-\frac{1}{24 \pi^{2}} \int_{M_{4}} \operatorname{Tr}(R \wedge R)+\frac{1}{24 \pi^{2}} \int_{\partial M_{4}} \operatorname{Tr}(\Pi \wedge R)-\eta\left(\partial M_{4}\right) .
$$

Here $\sigma\left(M_{4}\right)$ is the signature of $M_{4}, R$ is the curvature tensor of $M_{4}, \Pi$ is the second fundamental form of the boundary, and $\eta\left(\partial M_{4}\right)$ denotes the eta invariant ${ }^{15}$ of the boundary conformal structure on $\partial M_{4}$. Recall that the latter is defined in terms of the analytic continuation of the series

$$
\eta(s)=\sum_{\lambda \neq 0} \frac{\operatorname{sign} \lambda}{|\lambda|^{s}}
$$

where the summation is over non-zero eigenvalues $\lambda$ of the first order differential operator $B=(-1)^{p}(* \mathrm{~d}-\mathrm{d} *)$ acting on even forms $\Omega^{2 p}\left(\partial M_{4}\right)$. Specifically, one defines $\eta\left(\partial M_{4}\right)=$ $\eta(0)$, which may thus be thought of as a regularization of the number of positive eigenvalues of $B$ minus the number of negative eigenvalues. This is a conformal invariant of the boundary, but in general depends on the conformal class.

We may apply (4.23) in the case at hand [38] by noting that on a four-manifold

$$
\operatorname{Tr}(R \wedge R)=-2\left(\left|W^{+}\right|^{2}-\left|W^{-}\right|^{2}\right) \sqrt{\operatorname{det} g} \mathrm{~d}^{4} x,
$$

where $W^{ \pm}$denotes the self-dual/anti-self-dual parts of $W$. Moreover, the boundary term in (4.23) involving the second fundamental form $\Pi$ is zero; this follows because $\Pi$ is proportional to the boundary metric ${ }^{16}$ for the asymptotically locally Euclidean AdS boundary

\footnotetext{
${ }^{15}$ We hope that no confusion arises between this and the almost contact form on $M_{3}$, which we have also called $\eta$.

${ }^{16}$ That is, the boundary is totally umbilical.
} 
condition, and the trace is then zero on using the Bianchi identity for the curvature $R$. We thus conclude that

$$
\frac{1}{12 \pi^{2}} \int_{M_{4}}\left(\left|W^{+}\right|^{2}-\left|W^{-}\right|^{2}\right) \sqrt{\operatorname{det} g} \mathrm{~d}^{4} x=\sigma\left(M_{4}\right)+\eta\left(\partial M_{4}\right),
$$

When $\left(M_{4}, \mathrm{~d} s_{\mathrm{SDE}}^{2}\right)$ is anti-self-dual one can combine (4.26) with (4.22) to obtain (we have corrected a sign in [39])

$$
I^{\text {grav }}=\frac{3 \pi}{4 G_{4}} \eta\left(\partial M_{4}\right)+\frac{\pi}{4 G_{4}}\left(2 \chi\left(M_{4}\right)+3 \sigma\left(M_{4}\right)\right) .
$$

This expresses the gravitational contribution to the free energy as a conformally invariant local contribution from the boundary $\partial M_{4}$, plus a purely topological part depending on the filling $M_{4}$.

In the supergravity setting there is also the gauge field contribution $I^{F}$ to the action. Given that the Killing spinor is charged under the graviphoton field $A$, the natural operator on $M_{4}$ to consider is the index of the associated twisted Dirac operator $\mathcal{D}_{A}$. The index theorem in this case reads

$$
\begin{aligned}
\operatorname{Ind}_{\mathcal{D}_{\mathcal{A}}}= & \frac{1}{24 \cdot 8 \pi^{2}} \int_{M_{4}} \operatorname{Tr}(R \wedge R)-\frac{1}{24 \cdot 8 \pi^{2}} \int_{\partial M_{4}} \operatorname{Tr}(\Pi \wedge R)-\frac{1}{8 \pi^{2}} \int_{M_{4}} F \wedge F \\
& -\frac{1}{2}\left(\eta_{\mathcal{D}_{A}}\left(\partial M_{4}\right)+h_{\mathcal{D}_{A}}\left(\partial M_{4}\right)\right) .
\end{aligned}
$$

Here $\operatorname{Ind}_{\mathcal{D}_{\mathcal{A}}}$ is the index of the Dirac operator on $M_{4}$, twisted by the graviphoton $A$, with APS boundary conditions. The eta invariant is defined analogously to (4.24), replacing the operator $B$ by the restriction of the Dirac operator to the boundary, while $h_{\mathcal{D}_{A}}\left(\partial M_{4}\right)$ is the number of zero modes for that operator. As for the signature operator, the boundary term in (4.28) involving the second fundamental form is zero, and we thus find the total holographic free energy $I=I^{\text {grav }}+I^{F}$ may be written

$$
I=\frac{\pi}{2 G_{4}}\left\{\left[\eta_{\mathcal{D}_{A}}\left(\partial M_{4}\right)+h_{\mathcal{D}_{A}}\left(\partial M_{4}\right)+\frac{7}{4} \eta\left(\partial M_{4}\right)\right]+\left[\chi\left(M_{4}\right)+2 \operatorname{Ind}_{\mathcal{D}_{A}}+\frac{7}{4} \sigma\left(M_{4}\right)\right]\right\} .
$$

Here the terms in the first square bracket depend only on the conformal boundary, via eta invariants of the boundary twisted Dirac operator and signature operator, while the terms in the second square bracket are topological invariants of $M_{4}$ (each of $\chi\left(M_{4}\right), \sigma\left(M_{4}\right)$ and $\operatorname{Ind}_{\mathcal{D}_{A}}$ is of course an integer).

Finally, as a simple corollary of our results notice that we obtain a formula for the eta invariant of $M_{3} \cong S^{3}$, arising as the conformal boundary of a toric self-dual Einstein metric on the ball:

$$
\eta\left(M_{3}\right)=\frac{\left|b_{1}+b_{2}\right|^{2}}{3 b_{1} b_{2}}-\frac{2}{3}+\frac{1}{192 \pi^{2}} \int_{M_{3}}\left(3 w_{(1)}^{3}+4 w_{(1)} w_{(2)}\right) \sqrt{\operatorname{det} g_{M_{3}}} \mathrm{~d}^{3} x .
$$

For example, in section 5.2 below we will see how the general formulae derived thus far apply when one takes the self-dual Einstein metric on the four-ball to be the Euclidean Taub-NUT-AdS metric. In this case the conformal boundary is a biaxially squashed threesphere. One can then use (4.30) to compute the $\eta$ invariant of this conformal geometry to obtain $\eta=-\frac{2}{3}\left(1-4 s^{2}\right)^{2}$, where $s$ is the squashing parameter of section 5.2. This agrees with a direct computation of the eta invariant in [40]. 


\section{$5 \quad$ Examples}

In this section we illustrate our general results by discussing three explicit families of solutions. These consist of three sets of self-dual Einstein metrics on the four-ball, studied previously by some of the authors in $[6,8-10]$. We begin with $\mathrm{AdS}_{4}$ in section 5.1. Although the metric is trivial, the one-parameter family of instantons given by our general results is non-trivial, and it turns out that this family is identical to that in [6]. The solutions in sections 5.2 and 5.3 each add a deformation parameter, meaning that the metrics in each subsequent section generalize that in the previous section. Particular supersymmetric instantons on these backgrounds were found in [8-10], but our general results allow us to study the most general choice of instanton, leading to new solutions. Furthermore, in section 5.4 we indicate how to generalize these metrics further by adding an arbitrary number of parameters. This is discussed in more detail in appendix $\mathrm{C}$.

\section{$5.1 \quad \mathrm{AdS}_{4}$}

The metric on Euclidean $\mathrm{AdS}_{4}$ can be written as

$$
\mathrm{d} s_{\mathrm{EAdS}_{4}}^{2}=\frac{\mathrm{d} q^{2}}{q^{2}+1}+q^{2}\left(\mathrm{~d} \vartheta^{2}+\cos ^{2} \vartheta \mathrm{d} \varphi_{1}^{2}+\sin ^{2} \vartheta \mathrm{d} \varphi_{2}^{2}\right)
$$

Here $q$ is a radial variable with $q \in[0, \infty)$, so that the NUT is at $q=0$ while the conformal boundary is at $q=\infty$. The coordinate $\vartheta \in\left[0, \frac{\pi}{2}\right]$, with the endpoints being the two axes of $\mathbb{R}^{2} \oplus \mathbb{R}^{2} \cong \mathbb{R}^{4}$. The $\mathrm{AdS}_{4}$ metric is of course both self-dual and anti-self-dual.

Writing a general choice of Reeb vector field as $K=b_{1} \partial_{\varphi_{1}}+b_{2} \partial_{\varphi_{2}}$, as in our general discussion (3.32), the function $y$ is then defined in terms of $K$ via (3.33) and (3.34). Using these formulae one easily computes

$$
y(q, \vartheta)=\frac{1}{\sqrt{\left(b_{2}+b_{1} \sqrt{q^{2}+1}\right)^{2} \cos ^{2} \vartheta+\left(b_{1}+b_{2} \sqrt{q^{2}+1}\right)^{2} \sin ^{2} \vartheta}} .
$$

Notice that indeed $y_{\mathrm{NUT}}=1 /\left|b_{1}+b_{2}\right|$, in agreement with (3.36). Using (5.2) one can also verify the general behaviour in section 3.4 very explicitly. In particular we see the very different global behaviour, depending on the sign of $b_{1} / b_{2}$. If $b_{1} / b_{2}>0$ then $1 / y$ is nowhere zero, while if $b_{1} / b_{2}<0$ instead $1 / y$ has a zero on $M_{4}$. More precisely, if $-1<b_{1} / b_{2}<0$ then $1 / y=0$ at $\left\{\vartheta=0, q=\sqrt{b_{2}^{2}-b_{1}^{2}} /\left|b_{1}\right|\right\}$, while if $b_{1} / b_{2}<-1$ then $1 / y=0$ at $\{\vartheta=$ $\left.\frac{\pi}{2}, q=\sqrt{b_{1}^{2}-b_{2}^{2}} /\left|b_{2}\right|\right\}$. These are each a copy of $S^{1}$ at one or other of the "axes" of $\mathbb{R}^{2} \oplus \mathbb{R}^{2}$, at the corresponding radius given by $q$. In the special case that $b_{1}=-b_{2}$ we have $1 / y=0$ at the NUT itself, where the axes meet. These comments of course all agree with the general analysis in section 3.4, except here all formulae can be made completely explicit.

We thus indeed obtain smooth solutions for all $b_{1} / b_{2}>0$, as well as the isolated nonsingular solution with $b_{1} / b_{2}=-1$. In fact it is not difficult to check that the former are precisely the solutions first found in [6], where the parameter $b^{2}=b_{2} / b_{1}$ (compare to the formulae at the beginning of section 2.5 of [6]). To see this we may compute the instanton 
using the formulae in section 2, finding

$$
A=\frac{\left(b_{1}+b_{2} \sqrt{q^{2}+1}\right) \mathrm{d} \varphi_{1}+\left(b_{2}+b_{1} \sqrt{q^{2}+1}\right) \mathrm{d} \varphi_{2}}{2 \sqrt{\left(b_{2}+b_{1} \sqrt{q^{2}+1}\right)^{2} \cos ^{2} \vartheta+\left(b_{1}+b_{2} \sqrt{q^{2}+1}\right)^{2} \sin ^{2} \vartheta}},
$$

which agrees with the corresponding formula in [6]. In particular one can check that this gives a regular instanton when $b_{1} / b_{2}>0$, with the particular cases that $b_{1} / b_{2}= \pm 1$ giving a trivial instanton, and correspondingly the conformal Kähler structure is flat. We shall comment further on this below. Moreover, one can also check that the singular instantons with $b_{1} / b_{2}<0$ are singular at precisely the locus that $1 / y=0$, again in agreement with our general discussion.

In this case we may also compute all other functions appearing in sections 2, 3 and 4 explicitly. For example, we find

$$
V(q, \vartheta)=\frac{\left(b_{2}+b_{1} \sqrt{q^{2}+1}\right)^{2} \cos ^{2} \vartheta+\left(b_{1}+b_{2} \sqrt{q^{2}+1}\right)^{2} \sin ^{2} \vartheta}{q^{2}\left(b_{1}^{2} \cos ^{2} \vartheta+b_{2}^{2} \sin ^{2} \vartheta\right)},
$$

while the functions $w_{(1)}$ and $w_{(2)}$ on $\partial M_{4}=M_{3} \cong S^{3}$ appearing in the free energy computations are given by

$$
w_{(1)}=\frac{-4 b_{1} b_{2}}{\sqrt{b_{1}^{2} \cos ^{2} \vartheta+b_{2}^{2} \sin ^{2} \vartheta}}, \quad w_{(2)}=\frac{-2\left(3 b_{1}^{2} b_{2}^{2}+b_{1}^{4} \cos ^{2} \vartheta+b_{2}^{4} \sin ^{2} \vartheta\right)}{b_{1}^{2} \cos ^{2} \vartheta+b_{2}^{2} \sin ^{2} \vartheta} .
$$

Using these expressions one can verify all of the key formulae in our general analysis. For example, the integrals in (3.39), (4.8), (4.16) and (4.19) are all easily computed in closed form.

Finally, let us return to discuss the special cases $b_{1} / b_{2}= \pm 1$, where recall that the instanton is trivial and the conformal Kähler structure is flat. The latter is thus locally the flat Kähler metric on $\mathbb{C}^{2}$, but in fact in the two cases $b_{1} / b_{2}= \pm 1$ the Euclidean $\mathrm{AdS}_{4}$ metric is conformally embedded into different regions of $\mathbb{C}^{2}$. Notice this has to be the case, because the conformal factor $y$ of the $b_{1} / b_{2}=+1$ solution has $y_{\mathrm{NUT}}=1 /\left(2\left|b_{1}\right|\right)$, while for the $b_{1} / b_{2}=-1$ solution instead $y_{\mathrm{NUT}}=\infty$. We may see this concretely by writing the flat Kähler metric on $\mathbb{C}^{2}$ as

$$
\mathrm{d} s_{\text {flat }}^{2}=\mathrm{d} R^{2}+R^{2}\left(\mathrm{~d} \vartheta^{2}+\cos ^{2} \vartheta \mathrm{d} \varphi_{1}^{2}+\sin ^{2} \vartheta \mathrm{d} \varphi_{2}^{2}\right) .
$$

In both cases the change of radial coordinate to (5.1) is

$$
q(R)=\frac{2 R}{\left|R^{2}-1\right|} .
$$

However, for the $b_{1} / b_{2}=+1$ case the range of $R$ is $0 \leq R<1$, with the NUT being at $R=0$ and the conformal boundary being at $R=1$; while for the $b_{1} / b_{2}=-1$ case the range of $R$ is instead $1<R \leq \infty$, with the NUT being at $R=\infty$ (and the conformal boundary again being at $R=1$ ). In particular the two conformal factors are

$$
y(R)=\frac{1}{2\left|b_{1}\right|}\left|R^{2}-1\right| .
$$


The two solutions $b_{1} / b_{2}= \pm 1$ thus effectively fill opposite sides of the unit sphere in $\mathbb{C}^{2}$, and because of this they induce opposite orientations on $S^{3}$. Again, this may be seen rather explicitly in various formulae. For example, $w_{(1)}=\mp 4\left|b_{1}\right|$ in the two cases, so that the boundary Killing spinor equation (3.23) on the round $S^{3}$ becomes respectively $\nabla_{i}^{(3)} \chi=\mp \frac{i}{2}\left|b_{1}\right| \gamma_{i} \chi$, where one can take the gamma matrices to be the Pauli matrices $\gamma_{i}=\sigma_{i}$ in an orthonormal frame.

\subsection{Taub-NUT-AdS 4}

The Taub-NUT-AdS 4 metrics are a one-parameter family of self-dual Einstein metrics on the four-ball, and have been studied in detail in $[8,9]$. The metric may be written as

$$
\mathrm{d} s_{4}^{2}=\frac{r^{2}-s^{2}}{\Omega(r)} \mathrm{d} r^{2}+\left(r^{2}-s^{2}\right)\left(\tau_{1}^{2}+\tau_{2}^{2}\right)+\frac{4 s^{2} \Omega(r)}{r^{2}-s^{2}} \tau_{3}^{2},
$$

where

$$
\Omega(r)=(r \mp s)^{2}[1+(r \mp s)(r \pm 3 s)],
$$

and $\tau_{1}, \tau_{2}, \tau_{3}$ are left-invariant one-forms on $\mathrm{SU}(2) \simeq S^{3}$. The latter may be written in terms of Euler angular variables as

$$
\tau_{1}+\mathrm{i} \tau_{2}=\mathrm{e}^{-\mathrm{i} \varsigma}(\mathrm{d} \theta+\mathrm{i} \sin \theta \mathrm{d} \varphi), \quad \tau_{3}=\mathrm{d} \varsigma+\cos \theta \mathrm{d} \varphi .
$$

Here $\varsigma$ has period $4 \pi$, while $\theta \in[0, \pi]$ with $\varphi$ having period $2 \pi$. The radial coordinate $r$ lies in the range $r \in\left[s, \infty\right.$ ), with the NUT (origin of the ball $\cong \mathbb{R}^{4}$ ) being at $r=s$. The parameter $s>0$ is referred to as the squashing parameter, with $s=\frac{1}{2}$ being the Euclidean $\mathrm{AdS}_{4}$ metric studied in the previous section. Indeed, the metric is asymptotically locally Euclidean AdS as $r \rightarrow \infty$, with

$$
\mathrm{d} s_{4}^{2} \approx \frac{\mathrm{d} r^{2}}{r^{2}}+r^{2}\left(\tau_{1}^{2}+\tau_{2}^{2}+4 s^{2} \tau_{3}^{2}\right)
$$

so that the conformal boundary at $r=\infty$ is a biaxially squashed $S^{3}$.

Using the results of this paper we may write a general choice of Reeb vector field as $K=\left(b_{1}+b_{2}\right) \partial_{\varphi}+\left(b_{1}-b_{2}\right) \partial_{\varsigma}$, as in our general discussion (3.32), and the function $y$ is then defined in terms of $K$ via (3.33) and (3.34). Using these one computes

$$
\begin{aligned}
\frac{1}{y(r, \theta)^{2}}= & {\left[2\left(b_{1}-b_{2}\right)(r-s) s+\left(b_{1}+b_{2}\right)(1+2(r-s) s) \cos \theta\right]^{2} } \\
& +\left(b_{1}+b_{2}\right)^{2}[1+(r-s)(r+3 s)] \sin ^{2} \theta
\end{aligned}
$$

Notice that indeed $y_{\mathrm{NUT}}=\lim _{r \rightarrow s} y(r, \theta)=1 /\left|b_{1}+b_{2}\right|$. We see that if $b_{1} / b_{2}>0$ or $b_{1} / b_{2}=$ -1 then $1 / y$ is indeed never zero (except at the NUT in the latter case), as expected. In this way we obtain a two-parameter family of regular supersymmetric solutions, parametrized by the squashing parameter $s$ and $b_{1} / b_{2}$. One can also compute explicitly the corresponding instanton $F$ for a general choice of $s$ and $b_{1} / b_{2}$, although in practice it turns out to be more convenient to derive this as a special limit of the Plebanski-Demianski solutions, discussed in section 5.3. We do this in appendix C.6, where the resulting expression for $F$ is given 
in (C.111). In the remainder of this subsection we shall instead discuss further some special cases, making contact with the previous results $[8,9]$.

While the Taub-NUT-AdS metric (5.9) has $\mathrm{SU}(2) \times \mathrm{U}(1)$ isometry, a generic choice of the Killing vector $K=\left(b_{1}+b_{2}\right) \partial_{\varphi}+\left(b_{1}-b_{2}\right) \partial_{\varsigma}$ breaks the symmetry of the full solution to $\mathrm{U}(1) \times \mathrm{U}(1)$. In particular, this symmetry is also broken by the corresponding instanton $A$. On the other hand, in $[8,9]$ the $\mathrm{SU}(2) \times \mathrm{U}(1)$ symmetry of the metric was also imposed on the gauge field, which results in two one-parameter subfamilies of the above two-parameter family of solutions, which are $1 / 4$ BPS and $1 / 2$ BPS, respectively. In each case this effectively fixes the Killing vector $K$ (or rather the parameter $b_{1} / b_{2}$ ) as a function of the squashing parameter $s$.

1/4 BPS solution. This solution is simple enough that it can be presented in complete detail. The coordinate transformation to the (2.4) form for the 1/4 BPS solution reads

$$
r-s=1 / y, \quad-2 s \tau_{3}=\mathrm{d} \psi+\phi,
$$

and

$$
y^{2}\left(r^{2}-s^{2}\right)=\mathrm{e}^{w} V\left(1+|z|^{2}\right)^{2}, \quad \frac{r^{2}-s^{2}}{\Omega(r)}=y^{2} V .
$$

Notice immediately that at the NUT $r=s$ we have $1 / y=0$, so that this solution must have $b_{1}=-b_{2}$ - we shall find this explicitly below. The metric $\left(\tau_{1}^{2}+\tau_{2}^{2}\right)$ is diffeomorphic to the Fubini-Study metric on $\mathbb{C P}^{1} \cong S^{2}$ :

$$
\tau_{1}^{2}+\tau_{2}^{2}=\frac{4 \mathrm{~d} z \mathrm{~d} \bar{z}}{\left(1+|z|^{2}\right)^{2}}
$$

The metric functions then simplify to

$$
V(y)=\frac{1+2 s y}{1+4 s y+y^{2}}, \quad w(y, z, \bar{z})=\log \frac{1+4 s y+y^{2}}{\left(1+|z|^{2}\right)^{2}},
$$

and it is straightforward to check these satisfy the defining equation (2.5) and Toda equation (2.7). The conformally related scalar-flat Kähler metric is

$$
\mathrm{d} s_{\text {Kahler }}^{2}=\frac{1+2 s y}{1+4 s y+y^{2}} \mathrm{~d} y^{2}+(1+2 s y)\left(\tau_{1}^{2}+\tau_{2}^{2}\right)+\frac{4 s^{2}\left(1+4 s y+y^{2}\right)}{1+2 s y} \tau_{3}^{2},
$$

with Kähler form

$$
\omega=-\mathrm{d} y \wedge 2 s \tau_{3}+(1+2 s y) \tau_{1} \wedge \tau_{2}=-\mathrm{d}\left[(1+2 s y) \tau_{3}\right] .
$$

Using the formula (2.8) for the gauge field $A$, we compute

$$
A=\frac{1}{2}\left(4 s^{2}-1\right) \frac{r-s}{r+s} \tau_{3}+\text { pure gauge, }
$$

which we see reproduces the $1 / 4$ BPS choice of instanton in section 3.3 of [9]. ${ }^{17}$ The supersymmetric Killing vector is $K=\partial_{\psi}=-\frac{1}{2 s} \partial_{\varsigma}$ and so generates the Hopf fibration of

\footnotetext{
${ }^{17}$ Notice that in [9] the opposite orientation convention was chosen, so that that instanton in [9] is selfdual, rather than anti-self-dual. Recall also from the discussion above equation (2.8) that the overall sign of the instanton is correlated with the sign of the supersymmetric Killing vector $K$. Here $K=-\frac{1}{2 s} \partial_{\varsigma}$, which is minus the expression in [9], hence leading to the opposite sign for the instanton gauge field $A$.
} 
$S^{3}$. Since $\varsigma=\varphi_{1}-\varphi_{2}, \varphi=\varphi_{1}+\varphi_{2}$ we hence find

$$
b_{1}=-b_{2}=-\frac{1}{4 s},
$$

which using (1.2) yields

$$
I_{1 / 4 \mathrm{BPS}}=\frac{\pi}{2 G_{4}}
$$

This formula matches the result of section 5.4 of [9].

1/2 BPS solution. The Taub-NUT-AdS metric (5.9) also admits a 1/2 BPS solution [8, 9]. We hence have two linearly independent Killing spinors, which may be parametrized by an arbitrary choice of constant two-component spinor $\chi_{(0)}=\left(\begin{array}{l}p \\ q\end{array}\right) \in \mathbb{C}^{2} \backslash\{0\} .{ }^{18}$ The correspondong Killing vector is given by the unlikely expression

$$
\begin{aligned}
K= & \left(2 s+\sqrt{4 s^{2}-1}\right)\left[2 \operatorname{Im}\left[\mathrm{e}^{\mathrm{i} \varphi} \mathbf{p} \overline{\mathbf{q}}\right] \partial_{\theta}+\left(|\mathrm{p}|^{2}-|\mathbf{q}|^{2}+2 \operatorname{Re}\left[\mathrm{e}^{\mathrm{i} \varphi} \mathrm{p} \overline{\mathbf{q}}\right] \cot \theta\right) \partial_{\varphi}\right] \\
& \left.+\left[\left(|\mathbf{p}|^{2}+|\mathbf{q}|^{2}\right)\left(\frac{1}{2 s}-2 s-\sqrt{4 s^{2}-1}\right)\right)-2 \operatorname{Re}\left[\mathrm{e}^{\mathrm{i} \varphi} \mathbf{p} \overline{\mathbf{q}}\right]\left(2 s+\sqrt{4 s^{2}-1}\right) \csc \theta\right] \partial_{\varsigma} .
\end{aligned}
$$

Since multiplying $\chi_{(0)}$ by a non-zero complex number $\lambda \in \mathbb{C}^{*}$ simply rescales $K$ by $|\lambda|^{2}$, this leads to a $\mathbb{C P}^{1}$ family of choices of Killing vector $K$ in this case. Of course, the vector (5.23) is not toric for generic choice of $\chi_{(0)}$. Nevertheless, one can still compute the various geometric quantities in section 2 . In particular one can check that the formula (2.18) for the instanton gives

$$
A=s \sqrt{4 s^{2}-1} \frac{r-s}{r+s} \tau_{3}+\text { pure gauge },
$$

for any choice of $K$ in (5.23), which agrees with the expression in $[8,9]$. Notice that the instanton is invariant under the $\mathrm{SU}(2) \times \mathrm{U}(1)$ symmetry of the metric, even though a choice of Killing vector $K$ breaks this symmetry. Indeed, in this case the conformal factor $y=y(r, \theta)$ for toric solutions given by (5.13) depends non-trivially on both $r$ and $\theta$, thus also breaking the SU(2) symmetry of the underlying Taub-NUT-AdS metric. This is to be contrasted with the $1 / 4$ BPS solution, where instead (5.13) reduces simply to $y=y(r)=1 /(r-s)($ see $(5.14))$.

The toric choices of $K$ for these $1 / 2$ BPS solutions correspond to the poles of the $\mathbb{C P}^{1}$ parameter space. For example, choosing $\mathrm{p}=1, \mathrm{q}=0$ above gives

$$
K=\left(2 s+\sqrt{4 s^{2}-1}\right) \partial_{\varphi}+\left(\frac{1}{2 s}-2 s-\sqrt{4 s^{2}-1}\right) \partial_{\varsigma},
$$

so that

$$
b_{1}=\frac{1}{4 s}, \quad b_{2}=-\frac{1}{4 s}+2 s+\sqrt{4 s^{2}-1} .
$$

The free energy (1.2) is thus

$$
I=\frac{2 \pi s^{2}}{G_{4}}
$$

which of course matches the result obtained in section 4.4 of [9].

\footnotetext{
${ }^{18}$ The full Killing spinor is given by substituting this into the right hand side of (2.29) of [9].
} 


\subsection{Plebanski-Demianski}

The Taub-NUT-AdS metric has been extended to a two-parameter family of smooth selfdual Einstein metrics on the four-ball in [10], which lie in the Plebanski-Demianski class of local solutions [41] to Einstein-Maxwell theory. We will henceforth refer to the solution of [10] as "Plebanski-Demianski". The metric may be written as

$$
\mathrm{d} s_{\mathrm{PD}}^{2}=\frac{\mathcal{P}(q)}{q^{2}-p^{2}}\left(\mathrm{~d} \tau+p^{2} \mathrm{~d} \sigma\right)^{2}-\frac{\mathcal{P}(p)}{q^{2}-p^{2}}\left(\mathrm{~d} \tau+q^{2} \mathrm{~d} \sigma\right)^{2}+\frac{q^{2}-p^{2}}{\mathcal{P}(q)} \mathrm{d} q^{2}-\frac{q^{2}-p^{2}}{\mathcal{P}(p)} \mathrm{d} p^{2},
$$

where

$$
\mathcal{P}(x)=\left(x-p_{1}\right)\left(x-p_{2}\right)\left(x-p_{3}\right)\left(x-p_{4}\right) .
$$

The roots of the quartic $\mathcal{P}(x)$ can be expressed in terms of the two parameters of the solution, $\hat{a}$ and $v$, as

$$
\begin{array}{ll}
p_{1}=-\frac{1}{2}-\sqrt{1+\hat{a}^{2}-v^{2}}, & p_{3}=\frac{1}{2}-\hat{a}, \\
p_{2}=-\frac{1}{2}+\sqrt{1+\hat{a}^{2}-v^{2}}, & p_{4}=\frac{1}{2}+\hat{a} .
\end{array}
$$

The coordinate $p \in\left[p_{3}, p_{4}\right]$ is essentially a polar angle variable, while $q \in\left[p_{4}, \infty\right)$ plays the role of a radial coordinate, with the conformal boundary being at $q=\infty$. The NUT/origin of $\mathbb{R}^{4}$ is located at $p=p_{3}, q=p_{4}$. The Killing vectors $\partial_{\tau}, \partial_{\sigma}$ generate the $\mathrm{U}(1)^{2}$ torus symmetry of the solution, with the coordinates related to our standard $2 \pi$-period coordinates $\varphi_{1}, \varphi_{2}$ on $\mathrm{U}(1)^{2}$ via

$$
\begin{aligned}
\tau & =\frac{2 p_{3}^{2}}{\mathcal{P}^{\prime}\left(p_{3}\right)} \varphi_{1}-\frac{2 p_{4}^{2}}{\mathcal{P}^{\prime}\left(p_{4}\right)} \varphi_{2}, \\
\sigma & =-\frac{2}{\mathcal{P}^{\prime}\left(p_{3}\right)} \varphi_{1}+\frac{2}{\mathcal{P}^{\prime}\left(p_{4}\right)} \varphi_{2} .
\end{aligned}
$$

In order that the metric is smooth on the four-ball the parameters must obey $v^{2}>2|\hat{a}|$, with the $\hat{a}=0$ limit being the Taub-NUT-AdS metric of the previous section, and further setting $v=1$ one recovers Euclidean $\mathrm{AdS}_{4}$ (we refer the reader to [10] for further details).

It is straightforward, but tedious, to express the metric (5.28) in the form (2.4), with an arbitrary choice of toric Killing vector $K=b_{1} \partial_{\varphi_{1}}+b_{2} \partial_{\varphi_{2}}$. For the special case of the Killing vector/instanton in the solution of [10], we work out the change of coordinates explicitly towards the end of section C.4, cf. equations (C.76), (C.83)-(C.85).

In the $(\tau, \sigma)$ coordinates an arbitrary Killing vector may be written as

$$
K=b_{\tau} \partial_{\tau}+b_{\sigma} \partial_{\sigma}
$$

where

$$
b_{\tau}=\frac{2 p_{3}^{2}}{\mathcal{P}^{\prime}\left(p_{3}\right)} b_{1}-\frac{2 p_{4}^{2}}{\mathcal{P}^{\prime}\left(p_{4}\right)} b_{2}, \quad b_{\sigma}=-\frac{2}{\mathcal{P}^{\prime}\left(p_{3}\right)} b_{1}+\frac{2}{\mathcal{P}^{\prime}\left(p_{4}\right)} b_{2} .
$$


Using (3.33) and (3.34) one can calculate

$$
\begin{aligned}
\frac{1}{y(p, q)^{2}}= & \frac{1}{4} \frac{1}{\left(q^{2}-p^{2}\right)^{2}}\left\{\left[\left(\frac{2 \mathcal{P}(q)}{q-p}-\mathcal{P}^{\prime}(q)\right)\left(b_{\tau}+b_{\sigma} p^{2}\right)\right.\right. \\
& \left.\left.-\left(\frac{2 \mathcal{P}(p)}{q-p}+\mathcal{P}^{\prime}(p)\right)\left(b_{\tau}+b_{\sigma} q^{2}\right)\right]^{2}-4 b_{\sigma}^{2} \mathcal{P}(q) \mathcal{P}(p)(q+p)^{2}\right\} .
\end{aligned}
$$

Notice that this is a sum of two non-negative terms. Furthermore, these terms may vanish only when evaluated at the roots $p=p_{3}, p=p_{4}$ or $q=p_{4}$, which correspond to the axes of $\mathbb{R}^{4}=\mathbb{R}^{2} \oplus \mathbb{R}^{2}$. Let us calculate these limits:

$$
\begin{aligned}
& \lim _{p \rightarrow p_{3}} \frac{1}{y^{2}}=\left(\frac{\left(b_{1}+b_{2}\right) v^{2}+2 \hat{a} b_{1}+b_{2}(2 q-1)}{v^{2}+2 \hat{a}}\right)^{2}, \\
& \lim _{p \rightarrow p_{4}} \frac{1}{y^{2}}=\left(\frac{\left(b_{1}+b_{2}\right) v^{2}-2 \hat{a} b_{2}+b_{1}(2 q-1)}{v^{2}-2 \hat{a}}\right)^{2}, \\
& \lim _{q \rightarrow p_{4}} \frac{1}{y^{2}}=\left(\frac{\left(b_{1}+b_{2}\right) v^{2}-2 \hat{a} b_{2}+b_{1}(2 p-1)}{v^{2}-2 \hat{a}}\right)^{2} .
\end{aligned}
$$

A careful analysis of the above limits shows that $1 / y$ does not vanish, and hence the metric is regular, whenever $b_{1} / b_{2}>0$, while $1 / y=0$ only at the NUT when $b_{1} / b_{2}=-1$. On the other hand, the the solution is indeed singular if $b_{1} / b_{2}<0$ and $b_{1} / b_{2} \neq-1$. Notice that we also easily recover the formula (3.36) for the conformal factor at the NUT: $\lim _{p \rightarrow p_{3}, q \rightarrow p_{4}} y=1 /\left|b_{1}+b_{2}\right|$.

In [10] particular supersymmetric instantons (particular choices of $b_{1} / b_{2}$ for fixed $\hat{a}$ and $v$ ) were studied for this two-parameter family of metrics, which by construction lie within the Plebanski-Demianski ansatz. The results of this paper extend these results to a general choice of instanton on the same background, parametrized by $b_{1} / b_{2}$, leading to a three-parameter family of regular supersymmetric solutions. The general expression for this instanton is lengthy, but computable, and the interested reader may find the details in appendix C.5.

\subsection{Infinite parameter generalization}

In each subsection we have generalized the metrics of the previous subsection by adding a parameter, and one might wonder whether one can find more general self-dual Einstein metrics on the four-ball. In fact from the gauge-gravity point of view it is more natural to ask the question of which conformal structures on $S^{3}$ may be filled by a self-dual Einstein metric. Of course one expects this problem to be overdetermined, and some general results in this direction appear in [42]. Roughly speaking, as long as the conformal class of the boundary metric $\left[g_{S^{3}}\right]$ is sufficiently close to the round metric $\left[g_{S^{3}}^{0}\right]$, then one can write $\left[g_{S^{3}}\right]=\left[g_{S^{3}}^{0}\right]+\left[g_{S^{3}}^{+}\right]+\left[g_{S^{3}}^{-}\right]$, where $\left[g_{S^{3}}^{0}\right]+\left[g_{S^{3}}^{ \pm}\right]$bound self-dual/anti-self-dual Einstein metrics on the four-ball $B^{4}$, respectively. Equivalently, viewed as self-dual fillings these induce opposite orientations on $S^{3}$. This may be regarded as a generalization of the well-known result of Fefferman-Graham [43] to the self-dual case. Another important general result 
is that these fillings are unique: that is, two self-dual Einstein four-manifolds $\left(M_{4}^{(1)}, g^{(1)}\right)$, $\left(M_{4}^{(2)}, g^{(2)}\right)$ inducing the same conformal structure on $M_{3}=\partial M_{4}$ are isometric [44].

However, starting with a particular (conformal) three-metric and trying to construct a global filling explicitly is likely to be very difficult. In order to construct further explicit examples one might instead attempt to directly generalize the Plebanski-Demianski metrics of the previous subsection. A natural way to do this is explained in more detail in appendix C. Specifically, in [26] the authors studied the general local geometry of toric selfdual Einstein metrics, which thus includes all the solutions (locally) above. In appropriate coordinates ${ }^{19}$ the metric takes the form

$$
\begin{aligned}
\mathrm{d} s_{\text {toric }}^{2}= & \frac{4 \rho^{2}\left(\mathcal{F}_{\rho}^{2}+\mathcal{F}_{\eta}^{2}\right)-\mathcal{F}^{2}}{4 \mathcal{F}^{2}} \mathrm{~d} s_{\mathcal{H}^{2}}^{2}+\frac{4}{\mathcal{F}^{2}\left(4 \rho^{2}\left(\mathcal{F}_{\rho}^{2}+\mathcal{F}_{\eta}^{2}\right)-\mathcal{F}^{2}\right)}\left[\left(y_{\rho}^{\mathrm{can}} \mathrm{d} \nu\right.\right. \\
& \left.\left.+\left(\eta y_{\rho}^{\mathrm{can}}-\rho y_{\eta}^{\mathrm{can}}\right) \mathrm{d} \varphi\right)^{2}+\left(y_{\eta}^{\mathrm{can}} \mathrm{d} \nu+\left(\rho y_{\rho}^{\mathrm{can}}+\eta y_{\eta}^{\mathrm{can}}-y^{\mathrm{can}}\right) \mathrm{d} \varphi\right)^{2}\right] .
\end{aligned}
$$

where we have defined

$$
y^{\mathrm{can}}(\rho, \eta) \equiv \sqrt{\rho} \mathcal{F}(\rho, \eta)
$$

and

$$
\mathrm{d} s_{\mathcal{H}^{2}}^{2}=\frac{\mathrm{d} \rho^{2}+\mathrm{d} \eta^{2}}{\rho^{2}}
$$

is the metric on hyperbolic two-space $\mathcal{H}^{2}$, regarded as the upper half plane with boundary at $\rho=0$. The metric (5.36) is entirely determined by the choice of function $\mathcal{F}=\mathcal{F}(\rho, \eta)$, and the metric is self-dual Einstein if and only if this solves the eigenfunction equation

$$
\Delta_{\mathcal{H}^{2}} \mathcal{F}=\frac{3}{4} \mathcal{F} \quad \Longleftrightarrow \quad \mathcal{F}_{\rho \rho}+\mathcal{F}_{\eta \eta}=\frac{3}{4 \rho^{2}} \mathcal{F},
$$

where $\mathcal{F}_{\rho} \equiv \partial_{\rho} \mathcal{F}$, etc. Unlike the Toda equation (2.7) this is linear, and one may add solutions. In particular there is a basic solution

$$
\mathcal{F}(\rho, \eta ; \lambda)=\frac{\sqrt{\rho^{2}+(\eta-\lambda)^{2}}}{\sqrt{\rho}},
$$

where $\lambda$ is any constant. Via linearity then

$$
\mathcal{F}(\rho, \eta)=\sum_{i=1}^{m} \alpha_{i} \mathcal{F}\left(\rho, \eta ; \lambda_{i}\right),
$$

also solves (5.39), for arbitrary constants $\alpha_{i}, \lambda_{i}, i=1, \ldots, m$. We refer to (5.41) as an $m$ pole solution. Of course, one could also replace the sum in (5.41) by an integral, smearing the monopoles in some chosen charge distribution.

Thus the local construction of toric self-dual Einstein metrics is very straightforward - the above gives an infinite-dimensional space. However, understanding when the above metrics extend to complete asymptotically locally hyperbolic metrics on a ball (or indeed

\footnotetext{
${ }^{19}$ Below, and in appendix C, $\eta$ is a coordinate. We hope that no confusion arises between this, the almost contact form on $M_{3}$, and the $\eta$ invariant. The latter uses will not appear in the remainder of the paper.
} 
any other topology for $M_{4}$ ) is more involved. In appendix $\mathrm{C}$ we take some steps in this direction by showing that the general 2-pole solution is simply (Euclidean) $\mathrm{AdS}_{4}$, while the general 3-pole solution is precisely the Plebanski-Demianski solutions of section 5.3. This requires taking into account the symmetries of (5.36) (in particular the PSL $(2, \mathbb{R})$ symmetry of $\mathcal{H}^{2}$ ), and then making a number of rather non-trivial coordinate transformations. We also analyse in detail the global structure of Euclidean $\mathrm{AdS}_{4}$ in the $(\rho, \eta)$ coordinates, together with some global properties of the Plebanski-Demianski solutions in the $(\rho, \eta)$ coordinates.

Some work has also been done on global properties of the metrics (5.36) in [45], although the focus in that paper is on constructing complete asymptotically locally Euclidean scalar-flat Kähler metrics, which are conformal to (5.36). However, these have non-trivial Lens space boundaries $S^{3} / \Gamma$, and correspondingly the second Betti number $b_{2}=\operatorname{dim} H_{2}\left(M_{4}, \mathbb{R}\right)$ of the filling $M_{4}$ is non-zero (they contain "bolt $S^{2}$ s"). The corresponding complete self-dual Einstein metrics in Theorem B of that paper then also do not have the topology of the ball. Thus it remains an interesting open problem to understand when the general $m$-pole metrics extend to complete metrics on the ball. ${ }^{20}$

Finally, let us remark that in [30] Lebrun has constructed infinitely many self-dual Einstein metrics on the four-ball using twistor methods. This is essentially a deformation argument, where one starts with (the twistor space of) Euclidean $\mathrm{AdS}_{4}$, and perturbs the twistor space. However, as such this is rather more implicit than the toric metrics above, and in order to construct supersymmetric solutions one needs to ensure that the resulting self-dual Einstein metric has at least one Killing vector field. Nevertheless, this might be an alternative method for analysing regularity of the above $m$-pole solutions, at least in a neighbourhood of Euclidean $\mathrm{AdS}_{4}$ in parameter space.

\section{Conclusions}

The main result of this paper is the proof of the formula (1.2) for the holographically renormalized on-shell action in minimal four-dimensional supergravity. This result is analogous to the general formula for the volume functional of a toric Sasakian manifold in [46]. Indeed, the latter was also entirely determined by the Reeb vector field of the corresponding Sasakian manifold, and was later shown to agree with the large $N$ limit of the trial $a$ function in a dual four-dimensional field theory [47]. ${ }^{21}$ Moreover, we have provided a general construction of regular supersymmetric solutions of this theory, ${ }^{22}$ based on self-dual Einstein metrics on the four-ball equipped with a one-parameter family of instanton fields for the graviphoton. Specifically, if the self-dual Eintein metric admits $n$ parameters, our constuction produces an $(n+1)$-parameter family of solutions. We have shown that the renormalized on-shell action does not depend on the $n$ metric parameters, but only on this last "instanton parameter". This matches beautifully the field theory results of [20].

\footnotetext{
${ }^{20}$ At the end of reference [26] it is briefly noted that one can obtain regular $m$-pole metrics by deforming, for example, a given 3-pole solution. It would be interesting to examine the details of this deformation argument further.

${ }^{21} \mathrm{~A}$ similar general result, valid for the trial free energy of a three-dimensional field theory with $\mathrm{AdS}_{4}$ dual, was conjectured in [48].

${ }^{22}$ Of course, these uplift to solutions of eleven-dimensional supergravity using the results of [49].
} 
We have also shown how all the previous examples in the literature, as well as some new examples that we have presented, can be understood as arising from an infinite-dimensional family of local self-dual Einstein metrics with torus symmetry [26]. In section 5.4 we have suggested that using this family of local metrics, it should be possible to construct global asymptotically locally (Euclidean) AdS self-dual Einstein metrics on the four-ball, thus obtaining an infinite family of completely explicit metrics. It will be interesting to analyse these $m$-pole solutions in more detail.

In this paper we have achieved a rather general understanding of the gauge/gravity duality for supersymmetric asymptotically locally Euclidean $\mathrm{AdS}_{4}$ solutions. Nevertheless, there are a number of possible extensions of our work. First, it is possible to extend the matching of the free energy (1.2) for the class of self-dual backgrounds we have considered to other BPS observables. In particular in [50] the Wilson loop around an orbit of the Killing vector $K$ is shown to be BPS in the field theory, and may also be computed via localization. The gravity dual is an M2-brane wrapping a calibrated copy of the Mtheory circle in the internal space [51], and computing its renormalized action one finds an analogously simple formula to (1.2), namely

$$
\lim _{N \rightarrow \infty} \log \langle W\rangle=\frac{\left|b_{1}\right|+\left|b_{2}\right|}{2} \ell \cdot \log \langle W\rangle_{1}
$$

where $\langle W\rangle_{1}$ denotes the large $N$ limit of the Wilson loop on the round sphere/AdS $\mathrm{S}_{4}$, whose $\log$ scales as $N^{1 / 2}$, and $2 \pi \ell$ denotes the length of the orbit of $K$ (for example, such orbits always close over the poles of the $S^{3}$, where $\ell=1 /\left|b_{1}\right|$ or $\ell=1 /\left|b_{2}\right|$, respectively; notice that for these Wilson loops (6.1) is again a function only of $\left.\left|b_{1} / b_{2}\right|\right)$. Details of this computation are given in [50].

One might further generalize our results by relaxing one or more of the assumptions we have made. For example, remaining in the context of minimal gauged supergravity, it would be very interesting to investigate the more general class of supersymmetric, but non(anti-)self-dual solutions [28]. Several examples of such solutions were constructed in [8, 9], and these all turn out to have a bulk topology different from the four-ball. This suggests that self-duality and the topology of supersymmetric asymptotically $\mathrm{AdS}_{4}$ solutions are two related issues, and it would be desirable to clarify this. On the other hand, at present it is unclear to us what is the precise dual field theory implication of non-trivial two-cycles in the geometry, and therefore this direction is both challenging and interesting. Perhaps related to this, one of our main results is that a smooth toric self-dual Einstein metric on the four-ball with supersymmetric Killing vector $K=b_{1} \partial_{\varphi_{1}}+b_{2} \partial_{\varphi_{2}}$ gives rise to a smooth supersymmetric solution only if $b_{1} / b_{2}>0$ or $b_{1} / b_{2}=-1$. Specifically, for other choices of $b_{1} / b_{2}$ the conformal factor/Killing spinor are singular in the interior of the bulk. Nevertheless, the conformal boundary is smooth for all choices of $b_{1}, b_{2}$, and the question arises as to how to fill those boundaries smoothly within gauged supergravity. A natural conjecture is that these are filled with the non-self-dual solutions mentioned above.

Another assumption that should be straightforward to relax is in taking the gauge field $A$ to be real. In general, if $A$ is complex the existence of one (Euclidean) Killing spinor does not imply that the metric possesses any isometry [28]. However, we expect that if one 
requires the existence of two spinors of opposite R-charge, then there will be canonically defined Killing vectors, and therefore it should be possible to analyse the solutions with the techniques of this paper.

All the above extensions would be important conceptually, in order to address the issue of uniqueness of the filling of a given conformal boundary geometry. In fact, this could also motivate the study of this problem in a more general consistent truncation, or directly in eleven-dimensional supergravity.

Of course, in any of these more general set-ups a central issue will be to prove a generalized version of the formula (1.2) for the renormalized on-shell action. In this respect, some of the methods that we employed to derive this may be more amenable to generalization than others. For example, the expression (4.29), given in terms of boundary conformal invariants and bulk topological invariants, might extend to the class of non-self-dual metrics and/or non-ball topology. We also expect that some of the results of the present paper can be adapted to dimensions different from four. In particular, on the one hand it would be very nice to understand better the structure of the holographically renormalized on-shell action in five dimensions, and on the other hand, to enlarge the list of examples, extending the work of [14].

\section{Acknowledgments}

D. F. is supported by the Berrow Foundation. D. M. and J. L. are supported by the ERC Starting Grant N. 304806, "The Gauge/Gravity Duality and Geometry in String Theory". D. M. also acknowledges partial support from the STFC grant ST/J002798/1. J. F. S. is supported by a Royal Society University Research Fellowship.

\section{A Spin connection of the Kähler metric}

For the Kähler metric (2.9) in the frame (2.10) the spin connection reads

$$
\begin{aligned}
\hat{\omega}^{01}= & -\frac{\left(\partial_{y} w+y \partial_{y}^{2} w\right)}{4 V^{3 / 2}} \hat{e}^{1}+\frac{\mathrm{i} y \partial_{y}\left(\partial_{z}-\partial_{\bar{z}}\right) w}{8 V^{3 / 2} \mathrm{e}^{w / 2}} \hat{e}^{2}-\frac{y \partial_{y}\left(\partial_{z}+\partial_{\bar{z}}\right) w}{8 V^{3 / 2} \mathrm{e}^{w / 2}} \hat{e}^{3}, \\
\hat{\omega}^{02}= & -\frac{y \partial_{y}\left(\partial_{z}+\partial_{\bar{z}}\right) w}{8 V^{3 / 2} \mathrm{e}^{w / 2}} \hat{e}^{0}+\frac{\mathrm{i} y \partial_{y}\left(\partial_{z}-\partial_{\bar{z}}\right) w}{8 V^{3 / 2} \mathrm{e}^{w / 2}} \hat{e}^{1}+\frac{\left(\partial_{y} w+y \partial_{y}^{2} w\right)-2 V \partial_{y} w}{4 V^{3 / 2}} \hat{e}^{2}, \\
\hat{\omega}^{03}= & -\frac{\mathrm{i} y \partial_{y}\left(\partial_{z}-\partial_{\bar{z}}\right) w}{8 V^{3 / 2} \mathrm{e}^{w / 2}} \hat{e}^{0}-\frac{y \partial_{y}\left(\partial_{z}+\partial_{\bar{z}}\right) w}{8 V^{3 / 2} \mathrm{e}^{w / 2}} \hat{e}^{1}+\frac{\left(\partial_{y} w+y \partial_{y}^{2} w\right)-2 V \partial_{y} w}{4 V^{3 / 2}} \hat{e}^{3}, \\
\hat{\omega}^{12}= & -\hat{\omega}^{03} \\
\hat{\omega}^{13}= & \hat{\omega}^{02}, \\
\hat{\omega}^{23}= & -\frac{\left(\partial_{y} w-y\left(\partial_{y} w\right)^{2}-y \partial_{y}^{2} w\right)}{4 V^{3 / 2}} \hat{e}^{1}+\frac{\mathrm{i}\left[2 V\left(\partial_{z}-\partial_{\bar{z}}\right) w-y \partial_{y}\left(\partial_{z}-\partial_{\bar{z}}\right) w\right]}{8 V^{3 / 2} \mathrm{e}^{w / 2}} \hat{e}^{2} \\
& -\frac{2 V\left(\partial_{z}+\partial_{\bar{z}}\right) w-y \partial_{y}\left(\partial_{z}+\partial_{\bar{z}}\right) w}{8 V^{3 / 2} \mathrm{e}^{w / 2}} \hat{e}^{3} .
\end{aligned}
$$

Here we have used both (2.5) and (2.6). 


\section{B Weyl transformations of the boundary}

In section 3 of the main text we studied the boundary geometry and Killing spinor equation using the radial coordinate $r=1 / y$ defined naturally by supersymmetry. This gives a preferred representative for the conformal class of the boundary metric on $M_{3}$. In this appendix we study the more general choice $r=1 /(\Omega y)$, where $\Omega=\Omega(z, \bar{z})$ is an arbitrary smooth, basic, nowhere zero function on $M_{3}$. This results in a Weyl transformation of the boundary geometry and corresponding Killing spinor equation. We will see that we precisely recover the boundary structure, derived from a purely three-dimensional perspective, in [20, 25].

For comparison with [20], we begin by rescaling the constant-norm Kähler spinor $\zeta$ as

$$
\zeta \equiv \Omega^{-1 / 2}(z, \bar{z}) \hat{\zeta}
$$

so that the norm of $\hat{\zeta}$ is $\Omega^{1 / 2}$ if we normalize $\zeta$ to have unit norm. We then also have a rescaling of the four-dimensional Killing spinor $\epsilon$,

$$
\hat{\epsilon} \equiv \Omega^{1 / 2} \epsilon=\frac{1}{\sqrt{2 y}}\left(1+V^{-1 / 2} \hat{\Gamma}_{0}\right) \hat{\zeta} .
$$

Recall $\epsilon$ solves the Killing spinor equation (2.3), with the gauge field $A_{\mu}$ given by (2.8). Using instead $\hat{\epsilon}$ this Killing spinor equation reads

$$
\left(\nabla_{\mu}-\mathrm{i} A_{\mu}-\frac{1}{2} \partial_{\mu} \log \Omega+\frac{1}{2} \Gamma_{\mu}+\frac{\mathrm{i}}{4} F_{\nu \rho} \Gamma^{\nu \rho} \Gamma_{\mu}\right) \hat{\epsilon}=0,
$$

where the third term appears due to the rescaling. ${ }^{23}$

With the new choice of radial coordinate the boundary metric is

$$
\mathrm{d} s_{M_{3}}^{2}=\Omega^{2}(z, \bar{z})\left[\left(\mathrm{d} \psi+\phi_{0}\right)^{2}+4 \mathrm{e}^{w_{(0)}} \mathrm{d} z \mathrm{~d} \bar{z}\right] .
$$

As always, we introduce an orthonormal frame for this metric:

$$
e_{(3)}^{1}=\Omega\left(\mathrm{d} \psi+\phi_{0}\right), \quad e_{(3)}^{2}+\mathrm{i} e_{(3)}^{3}=2 \Omega \mathrm{e}^{w_{(0)} / 2} \mathrm{~d} z .
$$

The four-dimensional geometry is the same as before, namely

$$
\mathrm{d} s_{\mathrm{SDE}}^{2}=\frac{1}{y^{2}}\left[V^{-1}(\mathrm{~d} \psi+\phi)^{2}+V\left(\mathrm{~d} y^{2}+4 \mathrm{e}^{w} \mathrm{~d} z \mathrm{~d} \bar{z}\right)\right],
$$

and we will use the frame

$$
e^{0}=\frac{1}{y} V^{1 / 2} \mathrm{~d} y, \quad e^{1}=\frac{1}{y} V^{-1 / 2}(\mathrm{~d} \psi+\phi), \quad e^{2}+\mathrm{i} e^{3}=\frac{2}{y}\left(V \mathrm{e}^{w}\right)^{1 / 2} \mathrm{~d} z .
$$

Calculating the spin connection of (B.7), expanding in $y$ and comparing to the spin connection of (B.5), we find

$$
\begin{aligned}
\omega^{12} & =\omega_{(3)}^{12}-\partial_{2} \log \Omega e_{(3)}^{1}+\mathcal{O}(y), \\
\omega^{13} & =\omega_{(3)}^{13}-\partial_{3} \log \Omega e_{(3)}^{1}+\mathcal{O}(y), \\
\omega^{23} & =\omega_{(3)}^{23}-\partial_{3} \log \Omega e_{(3)}^{2}+\partial_{2} \log \Omega e_{(3)}^{3}+\mathcal{O}(y), \\
\omega^{0 i} & =\frac{1}{y} \Omega^{-1}\left(1+\frac{1}{4} y w_{(1)}\right) e_{(3)}^{i}+\mathcal{O}(y),
\end{aligned}
$$

\footnotetext{
${ }^{23}$ As this term is a total derivative it can formally be absorbed into a complex gauge transformation of $A_{\mu}$, although as we shall see all gauge fields will in the end be real.
} 
with $i=1,2,3$.

We next expand the Killing spinor equation with the rescaled spinor, $\hat{\epsilon}$. As in section 3 the term $\frac{\mathrm{i}}{4} F_{\nu \rho} \Gamma^{\nu \rho} \Gamma_{\mu}=\mathcal{O}(y)$ does not contribute. One gets

$$
\begin{aligned}
{\left[\nabla_{\mu}^{(3)}-\mathrm{i} A_{(0) \mu}-\right.} & \frac{1}{2} \partial_{\mu} \log \Omega+\frac{1}{2 y} \Omega^{-1}\left(1+\frac{1}{4} y w_{(1)}\right) e_{(3) \mu}^{i}\left(\Gamma_{i}-\Gamma_{i 0}\right) \\
& \left.-\frac{1}{2} \partial_{2} \log \Omega e_{(3) \mu}^{i} \Gamma_{i 2}-\frac{1}{2} \partial_{3} \log \Omega e_{(3) \mu}^{i} \Gamma_{i 3}+\mathcal{O}(y)\right] \hat{\epsilon}=0
\end{aligned}
$$

where $\mu=\psi, z, \bar{z}$, and $A_{(0) \mu}$ is the lowest order expansion of the gauge field (2.8), which in the frame (B.5) reads

$$
4 A_{(0)}=-\Omega^{-1} w_{(1)} e_{(3)}^{1}+\partial_{3} w_{(0)} e_{(3)}^{2}-\partial_{2} w_{(0)} e_{(3)}^{3} .
$$

The Killing spinor $\hat{\epsilon}$ expands as

$$
\hat{\epsilon}=\frac{1}{\sqrt{2 y}}\left[1+\Gamma_{0}+\frac{1}{4} y w_{(1)} \Gamma_{0}+\mathcal{O}\left(y^{2}\right)\right] \hat{\zeta}_{0},
$$

and when substituted into (B.9) gives a vanishing leading order term. The subleading term reads

$$
\begin{aligned}
& {\left[\left(\nabla_{i}^{(3)}-\mathrm{i} A_{(0) i}-\frac{1}{2} \partial_{i} \log \Omega\right)\left(1+\Gamma_{0}\right)-\frac{1}{8} w_{(1)} \Omega^{-1}\left(\Gamma_{i}-\Gamma_{i 0}\right)\right.} \\
& \left.-\frac{1}{2} \partial_{2} \log \Omega \Gamma_{i 2}\left(1+\Gamma_{0}\right)-\frac{1}{2} \partial_{3} \log \Omega \Gamma_{i 3}\left(1+\Gamma_{0}\right)\right] \hat{\zeta}_{0}=0 .
\end{aligned}
$$

The projection conditions (2.22) imply the following form for $\hat{\zeta}_{0}$,

$$
\hat{\zeta}_{0}=\left(\begin{array}{l}
\hat{\chi} \\
0
\end{array}\right) \quad \text { where } \quad \hat{\chi}=\left(\begin{array}{c}
\hat{\chi}_{0} \\
\hat{\chi}_{0}
\end{array}\right) .
$$

The three-dimensional Killing spinor equation then becomes

$$
\left[\nabla_{i}^{(3)}+\mathrm{i}\left(V_{i}-A_{i}^{(3)}\right)+\frac{1}{2} H \sigma_{i}+\frac{1}{2} \epsilon_{i j k} V_{j} \sigma_{k}\right] \hat{\chi}=0,
$$

with

$$
\begin{aligned}
H & =-\frac{\mathrm{i}}{4} w_{(1)} \Omega^{-1}+\mathrm{i} V_{1}, \quad A_{1}^{(3)}=A_{(0) 1}+\frac{3}{2} V_{1}, \\
A_{2}^{(3)} & =A_{(0) 2}-\frac{3}{2} \mathrm{i} V_{3}-\frac{3}{2} \mathrm{i} \partial_{2} \log \Omega+\frac{3}{2} \partial_{3} \log \Omega, \\
A_{3}^{(3)} & =A_{(0) 3}+\frac{3}{2} V_{3}, \\
V_{2}+\mathrm{i} V_{3} & =-\mathrm{i} \partial_{2} \log \Omega+\partial_{3} \log \Omega .
\end{aligned}
$$

The Killing spinor equation (B.14) is precisely of the form found in [25], which allows for the construction of supersymmetric field theories on $M_{3}$. The identifications of $A^{(3)}, V$ 
and $H$ are not unique because equation (B.14) has some symmetry properties, ${ }^{24} \mathrm{cf}$. (4.2) of [25]. In particular this gauge freedom allows one to freely choose $V_{1}$, as shown in (2.10) of [20]. Recall that $A_{(0)}$ is real. If we demand also the boundary gauge field $A^{(3)}$ to be real, one finds from the equations in (B.15) that also $V$ is real with

$$
V_{2}=\partial_{3} \log \Omega, \quad V_{3}=-\partial_{2} \log \Omega .
$$

This is exactly the result obtained for $V$ in [20] using the purely three-dimensional analysis of [25]. The remaining equations in (B.15) then further simplify to

$$
\begin{aligned}
H & =-\frac{\mathrm{i}}{4} w_{(1)} \Omega^{-1}+\mathrm{i} V_{1}, \\
A_{i}^{(3)} & =A_{(0) i}+\frac{3}{2} V_{i} .
\end{aligned}
$$

Again this is consistent with [20], where it was found (in our notation) that

$$
A_{\mu}^{(3)}=-\frac{\mathrm{i}}{2} H e_{(3) \mu}^{1}+V_{\mu}+j_{\mu},
$$

where

$$
j_{\mu}=\frac{\mathrm{i}}{4 \Omega^{2}}\left(s \partial_{\mu} \bar{s}-\bar{s} \partial_{\mu} s\right)+\frac{1}{2} \omega_{\mu(3)}^{23},
$$

and $|s|=\Omega$ is the square norm of the three-dimensional spinor,

$$
\hat{\chi}=\sqrt{s(\psi, z, \bar{z})}\left(\begin{array}{c}
\frac{1}{\sqrt{2}} \\
\frac{1}{\sqrt{2}}
\end{array}\right) .
$$

Hence we have $s=\Omega \mathrm{e}^{2 \mathrm{i} v(\psi, z, \bar{z})}$. Equation (B.20) then reads

$$
\begin{aligned}
j_{\mu} & =\partial_{\mu} v+\frac{1}{2} \omega_{\mu(3)}^{23} \\
& =\partial_{\mu} v-\frac{1}{8} \Omega^{-1} w_{(1)} e_{(3)}^{1}+\frac{1}{4}\left(\partial_{3} w_{(0)}+2 \partial_{3} \log \Omega\right) e_{(3)}^{2}-\frac{1}{4}\left(\partial_{2} w_{(0)}+2 \partial_{2} \log \Omega\right) e_{(3)}^{3}
\end{aligned}
$$

where we also used equation (2.6). Substituting equation (B.16), (B.17), and (B.22) into the right hand side of (B.19), this gives

$$
\begin{aligned}
A_{\mu}^{(3)} & =-\frac{1}{4} \Omega^{-1} w_{(1)} e_{(3) \mu}^{1}+\frac{1}{4} \partial_{3} w_{(0)} e_{(3) \mu}^{2}-\frac{1}{4} \partial_{2} w_{(0)} e_{(3) \mu}^{3}+\frac{3}{2} V_{\mu}+\partial_{\mu} v \\
& =A_{(0) \mu}+\frac{3}{2} V_{\mu}+\partial_{\mu} v
\end{aligned}
$$

where in the second line we used equation (B.10). As the last term in equation (B.23) is a total derivative, it can be absorbed into a gauge transformation of $A_{(0)}$. Thus we see that equation (B.19) reproduces (B.18) up to a gauge transformation. Indeed, such a gauge transformation with $v=\gamma \psi$ was shown in section 3.3 to be necessary in order for the gauge field to be globally well-defined on $M_{3} \cong S^{3}$.

\footnotetext{
${ }^{24}$ With an abuse of language, in this paper we refer to this symmetry as a "gauge" symmetry. Although $V$ is not a gauge field, and hence does not transform under gauge transformations. Hopefully this will not cause any confusion.
} 


\section{Toric self-dual Einstein metrics on the four-ball}

In this appendix we indicate how the metrics in section 5 may be extended to include arbitrarily many parameters, leaving further details of this construction for another occasion. The local form of these metrics was determined in [26], and is given in terms of so-called $m$-pole solutions. We discuss in detail the special cases of $m=2$ and $m=3$, showing that they correspond to Euclidean $\mathrm{AdS}_{4}$ and a particular metric discussed in [10]. The latter originates from a class of metrics originally studied by Plebanski-Demianski. Below we will also provide more details on the general instantons associated to a given self-dual Einstein metric, and a choice of Killing vector in the $\mathrm{U}(1)^{2}$ torus of isometries.

\section{C.1 Local form of the metrics and instanton}

Following [26], the local form of a toric self-dual Einstein metric can be written as ${ }^{25}$

$$
\begin{aligned}
\mathrm{d} s_{\text {toric }}^{2}= & \frac{4 \rho^{2}\left(\mathcal{F}_{\rho}^{2}+\mathcal{F}_{\eta}^{2}\right)-\mathcal{F}^{2}}{4 \mathcal{F}^{2}} \mathrm{~d} s_{\mathcal{H}^{2}}^{2}+\frac{4}{\mathcal{F}^{2}\left(4 \rho^{2}\left(\mathcal{F}_{\rho}^{2}+\mathcal{F}_{\eta}^{2}\right)-\mathcal{F}^{2}\right)}\left[\left(y_{\rho}^{\mathrm{can}} \mathrm{d} \nu\right.\right. \\
& \left.\left.+\left(\eta y_{\rho}^{\mathrm{can}}-\rho y_{\eta}^{\mathrm{can}}\right) \mathrm{d} \varphi\right)^{2}+\left(y_{\eta}^{\mathrm{can}} \mathrm{d} \nu+\left(\rho y_{\rho}^{\mathrm{can}}+\eta y_{\eta}^{\mathrm{can}}-y^{\mathrm{can}}\right) \mathrm{d} \varphi\right)^{2}\right] .
\end{aligned}
$$

Here we defined

$$
y^{\mathrm{can}}(\rho, \eta) \equiv \sqrt{\rho} \mathcal{F}(\rho, \eta),
$$

with $\mathcal{F}=\mathcal{F}(\rho, \eta)$ and the superscript "can" indicating that this is a canonical choice for the function $y$ (see below). We also have that

$$
\mathrm{d} s_{\mathcal{H}^{2}}^{2}=\frac{\mathrm{d} \rho^{2}+\mathrm{d} \eta^{2}}{\rho^{2}}
$$

is the metric on hyperbolic two-space $\mathcal{H}^{2}$, regarded as the upper half plane with boundary at $\rho=0$. Even though the metric (C.1) is local, this global description of $\mathcal{H}^{2}$ will be important. In particular, in the global construction of [45] the coordinate singularities along which Killing vectors vanish are mapped onto the boundary $\rho=0$ of $\mathcal{H}^{2}$, and we shall see this for the examples that we study below. The metric (C.1) is entirely determined by the choice of function $\mathcal{F}(\rho, \eta)$, and the metric is self-dual Einstein if and only if this solves the eigenfunction equation

$$
\Delta_{\mathcal{H}^{2}} \mathcal{F}=\frac{3}{4} \mathcal{F} \quad \Longleftrightarrow \quad \mathcal{F}_{\rho \rho}+\mathcal{F}_{\eta \eta}=\frac{3}{4 \rho^{2}} \mathcal{F},
$$

where $\mathcal{F}_{\rho} \equiv \partial_{\rho} \mathcal{F}$, etc. Crucially this is a linear equation, so we may add solutions as in the more familiar "multi-centre" types of solutions in other contexts.

As discussed in the main part of the paper, any self-dual Einstein metric with a choice of Killing vector gives rise to a conformal scalar-flat Kähler metric, with an associated conformal factor $y$. For the above metric (C.1) a natural canonical choice of Killing vector is $K=\partial_{\nu}$, and this leads to the associated conformal factor $y=y^{\text {can }}$ given by (C.2).

\footnotetext{
${ }^{25}$ We have reversed the sign of the metric $(1.1)$ in [26], so that for $4 \rho^{2}\left(\mathcal{F}_{\rho}^{2}+\mathcal{F}_{\eta}^{2}\right)-\mathcal{F}^{2}>0$ our metric (C.1) has Euclidean signature $(+,+,+,+)$ and negative scalar curvature.
} 


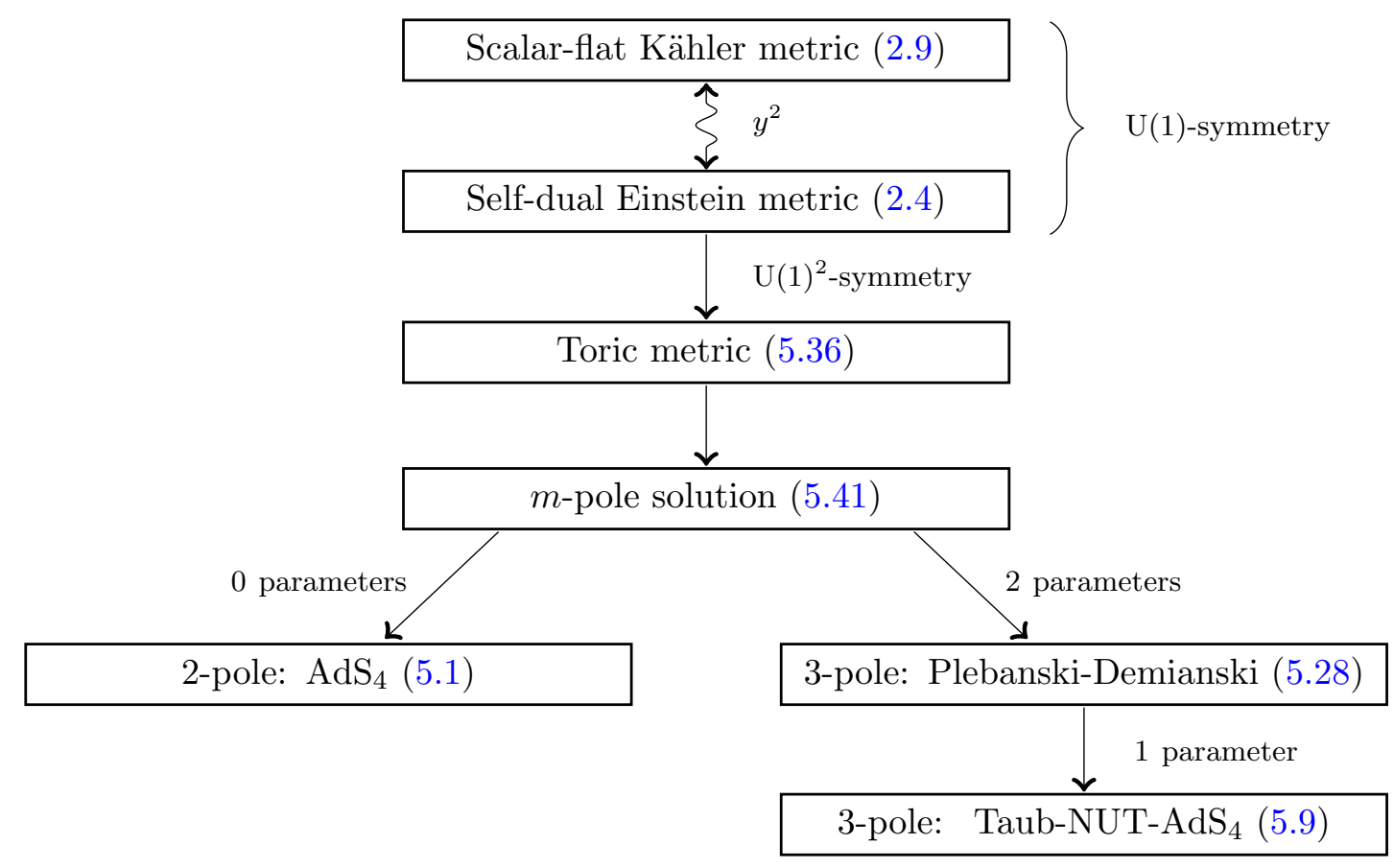

Figure 1. Overview of the metrics discussed in the main part of the paper and in the present appendix. The arrows point from a metric to a special case of the metric, except the wavy arrow which corresponds to a conformal transformation, i.e. equation (2.9).

Depending on global constraints, the Killing vector $\partial_{\nu}$ may have fixed points, and the associated supersymmetric solution may then be singular. However, we are also free to pick the supersymmetric Killing vector $K$ to be an arbitrary linear combination of Killing vectors

$$
K=b_{\nu} \partial_{\nu}+b_{\varphi} \partial_{\varphi}
$$

with real coefficients $b_{\nu}, b_{\varphi}$, giving the following $\left(b_{\nu}, b_{\varphi}\right)$-dependent conformal factor

$$
y(\rho, \eta)=\frac{\sqrt{\rho} \mathcal{F}(\rho, \eta)}{\sqrt{\left(b_{\nu}+b_{\varphi} \eta\right)^{2}+b_{\varphi}^{2} \rho^{2}}}=\frac{y^{\operatorname{can}}(\rho, \eta)}{\sqrt{\left(b_{\nu}+b_{\varphi} \eta\right)^{2}+b_{\varphi}^{2} \rho^{2}}} .
$$

Of course for $b_{\nu}=1$ and $b_{\varphi}=0$ one recovers $y=y^{\text {can }}$. It is simple to check that the conformally rescaled metric

$$
\mathrm{d} s^{2}=\frac{\rho \mathcal{F}^{2}(\rho, \eta)}{\left(b_{\nu}+b_{\varphi} \eta\right)^{2}+b_{\varphi}^{2} \rho^{2}} \mathrm{~d} s_{\text {toric }}^{2}
$$

is Kähler and scalar-flat.

A key feature of this construction will be that the conformal boundary $y^{\text {can }}=0$ will in general only be an implicit equation in the $(\rho, \eta)$ coordinates. However, these coordinates are well-suited for the discussion of regularity of the metric in the interior. The opposite is true in the $y$-coordinates given by supersymmetry in (2.4). Before discussing the general family of $m$-pole solutions, and the examples $m=2,3$, let us present the general explicit 
form of the instanton associated to the Killing vector (C.5). Using the following general formula for the instanton

$$
F=-\left(\frac{1}{2} y \mathrm{~d} K^{b}+y^{2} K^{b} \wedge J K^{b}\right)^{-}
$$

where $K^{b}$ denotes the one-form dual to the Killing vector $K$, and $J$ the complex structure (3.35), we compute

$$
\begin{aligned}
F= & \frac{y \mathcal{F}}{c_{11} \sqrt{\rho}\left(\mathcal{F}^{2}-4 \rho^{2}\left(\mathcal{F}_{\eta}^{2}+\mathcal{F}_{\rho}^{2}\right)\right)^{2}}\left[( g ^ { 1 3 } + g ^ { 2 4 } ) \left[2 c _ { 1 1 } \rho \left\{\mathcal{F}_{\rho}\left(2 b_{\varphi} \rho^{2} \mathcal{F}_{\eta}-\hat{\eta} \mathcal{F}\right)\right.\right.\right. \\
& \left.+2 \rho \mathcal{F}_{\rho \rho}\left(2 b_{\varphi} \rho^{2} \mathcal{F}_{\eta}-2 \hat{\eta} \rho \mathcal{F}_{\rho}-\hat{\eta} \mathcal{F}\right)+2 \rho^{2} \mathcal{F}_{\rho \eta}\left(b_{\varphi} \mathcal{F}-2 \hat{\eta} \mathcal{F}_{\eta}-2 b_{\varphi} \rho \mathcal{F}_{\rho}\right)\right\} \\
& \left.+c_{13} \hat{\eta}\left(\mathcal{F}^{2}-4 \rho^{2} \mathcal{F}_{\eta}^{2}\right)-8 c_{12} \rho^{2} \hat{\eta} \mathcal{F}_{\rho}^{2}\right]+\rho\left(g^{12}-g^{34}\right)\left[2 c _ { 1 1 } \left\{2 \rho ^ { 2 } \mathcal { F } _ { \rho \rho } \left(b_{\varphi} \mathcal{F}-2 b_{\varphi} \rho \mathcal{F}_{\rho}\right.\right.\right. \\
& \left.\left.-2 \hat{\eta} \mathcal{F}_{\eta}\right)+2 \rho \mathcal{F}_{\rho \eta}\left(\hat{\eta} \mathcal{F}+2 \rho \hat{\eta} \mathcal{F}_{\rho}-2 b_{\varphi} \rho^{2} \mathcal{F}_{\eta}\right)+3 b_{\varphi} \rho \mathcal{F} \mathcal{F}_{\rho}+4 \hat{\eta} \mathcal{F} \mathcal{F}_{\eta}+2 \rho \hat{\eta} \mathcal{F}_{\eta} \mathcal{F}_{\rho}\right\} \\
& \left.\left.-8 b_{\varphi}^{3} \rho^{4} \mathcal{F}_{\rho}^{2}-4 b_{\varphi} c_{13} \rho^{2} \mathcal{F}_{\eta}^{2}-b_{\varphi} c_{31} \mathcal{F}^{2}\right]\right]
\end{aligned}
$$

Here $y$ is given by the expression in (C.6) and we have defined $\hat{\eta} \equiv b_{\nu}+b_{\varphi} \eta$ and $c_{m n}=$ $c_{m n}(\rho, \eta) \equiv m \hat{\eta}^{2}+n b_{\varphi}^{2} \rho^{2}$. The vielbein are defined as

$$
\begin{aligned}
g^{1} & =\sqrt{\frac{4 \rho^{2}\left(\mathcal{F}_{\eta}^{2}+\mathcal{F}_{\rho}^{2}\right)-\mathcal{F}^{2}}{4 \rho^{2} \mathcal{F}^{2}}} \mathrm{~d} \eta \\
g^{2} & =\frac{2}{\sqrt{\mathcal{F}^{2}\left(4 \rho^{2}\left(\mathcal{F}_{\eta}^{2}+\mathcal{F}_{\rho}^{2}\right)-\mathcal{F}^{2}\right)}}\left(\left(\eta y_{\rho}^{\mathrm{can}}-\rho y_{\eta}^{\mathrm{can}}\right) \mathrm{d} \varphi+y_{\rho}^{\mathrm{can}} \mathrm{d} \nu\right), \\
g^{3} & =\frac{2}{\sqrt{\mathcal{F}^{2}\left(4 \rho^{2}\left(\mathcal{F}_{\eta}^{2}+\mathcal{F}_{\rho}^{2}\right)-\mathcal{F}^{2}\right)}}\left(\left(\eta y_{\eta}^{\mathrm{can}}+\rho y_{\rho}^{\mathrm{can}}-y^{\mathrm{can}}\right) \mathrm{d} \varphi+y_{\eta}^{\mathrm{can}} \mathrm{d} \nu\right), \\
g^{4} & =\sqrt{\frac{4 \rho^{2}\left(\mathcal{F}_{\eta}^{2}+\mathcal{F}_{\rho}^{2}\right)-\mathcal{F}^{2}}{4 \rho^{2} \mathcal{F}^{2}}} \mathrm{~d} \rho .
\end{aligned}
$$

\section{C.2 $m$-pole solutions}

There is a basic solution to (C.4), namely

$$
\mathcal{F}(\rho, \eta ; \lambda)=\frac{\sqrt{\rho^{2}+(\eta-\lambda)^{2}}}{\sqrt{\rho}},
$$

where $\lambda$ is any constant. We will refer to this as a single monopole solution, and via linearity then

$$
\mathcal{F}(\rho, \eta)=\sum_{i=1}^{m} \alpha_{i} \mathcal{F}\left(\rho, \eta ; \lambda_{i}\right),
$$

also solves (C.4), for arbitrary constants $\alpha_{i}, \lambda_{i}, i=1, \ldots, m$. We will refer to (C.12) as an $m$-pole solution. Of course, one could also replace the sum in (C.12) by an integral, 
smearing the monopoles in some chosen charge distribution. The local construction of infinitely many self-dual Einstein metrics is thus straightforward via this construction.

For the $m$-pole solution (C.12) the metric (C.1) depends on only $2 m-4$ of the $2 m$ constants in (C.12). This follows from taking into account symmetries. Recall that the isometry group of the hyperbolic upper half plane $\mathcal{H}^{2}$ is $\operatorname{PSL}(2, \mathbb{R})$. In terms of the $(\rho, \eta)$ coordinates in (C.3) this is generated by the three simple transformations

$$
\begin{array}{lll}
\text { Translation : } & \eta \rightarrow \eta+\mathbf{b}, & \\
\text { Rescaling : } & \eta \rightarrow \mu^{2} \eta, & \rho \rightarrow \mu^{2} \rho, \\
\text { Inversion : } & \eta \rightarrow-\frac{\eta}{\rho^{2}+\eta^{2}}, & \rho \rightarrow \frac{\rho}{\rho^{2}+\eta^{2}} .
\end{array}
$$

We may write these as $\mathrm{SL}(2, \mathbb{R})$ matrices by defining the complex coordinate $Z \equiv \eta+\mathrm{i} \rho$, so that $\operatorname{PSL}(2, \mathbb{R})$ acts as

$$
Z \rightarrow \frac{\mathbf{a} Z+\mathbf{b}}{\mathbf{c} Z+\mathbf{d}}, \quad\left(\begin{array}{ll}
\mathbf{a} & \mathbf{b} \\
\mathbf{c} & \mathbf{d}
\end{array}\right) \in \mathrm{SL}(2, \mathbb{R})
$$

The above three transformations are then

$$
T=\left(\begin{array}{cc}
1 & \mathbf{b} \\
0 & 1
\end{array}\right), \quad R=\left(\begin{array}{cc}
\mu & 0 \\
0 & \frac{1}{\mu}
\end{array}\right), \quad I=\left(\begin{array}{cc}
0 & -1 \\
1 & 0
\end{array}\right),
$$

respectively. The symmetries (C.13) extend to isometries of the self-dual Einstein metric (C.1) by also acting on the angular coordinates via

$$
\begin{array}{lll}
\text { Translation : } & \nu \rightarrow \nu-\mathbf{b} \varphi, & \\
\text { Rescaling : } & \nu \rightarrow \mu \nu, & \varphi \rightarrow \frac{1}{\mu} \varphi, \\
\text { Inversion : } & \nu \rightarrow-\varphi, & \varphi \rightarrow \nu,
\end{array}
$$

respectively, and for the $m$-pole solution (C.12) one acts on the monopole parameters $\alpha_{i}$, $\lambda_{i}$ via

$$
\begin{array}{lll}
\text { Translation : } & \lambda_{i} \rightarrow \lambda_{i}+\mathbf{b}, & \\
\text { Rescaling : } & \lambda_{i} \rightarrow \mu^{2} \lambda_{i}, & \alpha_{i} \rightarrow \frac{1}{\mu} \alpha_{i}, \\
\text { Inversion : } & \lambda_{i} \rightarrow-\frac{1}{\lambda_{i}}, & \alpha_{i} \rightarrow \alpha_{i}\left|\lambda_{i}\right|,
\end{array}
$$

respectively. Most of these are easily verified, apart from the action of inversion on the angular coordinates. Here it is useful to establish a number of transformation properties, such as $\eta \partial_{\rho}-\rho \partial_{\eta} \rightarrow-\eta \partial_{\rho}+\rho \partial_{\eta}$ under inversion. In addition to the above $\operatorname{PSL}(2, \mathbb{R})$ transformations, we may also simply rescale

$$
\mathcal{F}(\rho, \eta) \rightarrow \kappa \mathcal{F}(\rho, \eta), \quad \nu \rightarrow \kappa \nu, \quad \varphi \rightarrow \kappa \varphi,
$$

which for the $m$-pole solution simply scales $\alpha_{i} \rightarrow \kappa \alpha_{i}$. 
Note that under the $\operatorname{PSL}(2, \mathbb{R})$ symmetry action on the coordinates $(\rho, \eta)$, the basic monopole solution transforms as

$$
\alpha \frac{\sqrt{\rho^{2}+(\eta-\lambda)^{2}}}{\sqrt{\rho}} \rightarrow \alpha^{\prime} \frac{\sqrt{\rho^{2}+\left(\eta-\lambda^{\prime}\right)^{2}}}{\sqrt{\rho}}
$$

where

$$
\alpha^{\prime}=\alpha|\mathbf{c} \lambda+\mathbf{d}|, \quad \lambda^{\prime}=\frac{\mathbf{a} \lambda+\mathbf{b}}{\mathbf{c} \lambda+\mathbf{d}},
$$

with $\mathbf{a d}-\mathbf{b c}=1$. One can then use these symmetries to fix 4 of the $2 m$ parameters in (C.12). We shall see this explicitly for the 2-pole and 3-pole solutions that we examine in detail below.

\section{C.3 $\mathrm{AdS}_{4}$ from 2-pole solution}

The simplest example of the construction described above is the 2-pole solution, which turns out to be Euclidean $\mathrm{AdS}_{4}$, that is the four-dimensional hyperbolic space. Using the $\operatorname{PSL}(2, \mathbb{R})$ symmetry plus the overall scaling symmetry discussed in section C.2, we can set $\lambda_{1}=-\lambda_{2}=1$ and $\alpha_{1}=-\alpha_{2}=-\frac{1}{2}$ without loss of generality. Therefore we have

$$
\mathcal{F}_{\mathrm{EAdS}}=\frac{\sqrt{\rho^{2}+(\eta+1)^{2}}-\sqrt{\rho^{2}+(\eta-1)^{2}}}{2 \sqrt{\rho}}
$$

with the conformal factor for a generic choice of Killing vector

$$
y(\rho, \eta)=\frac{\sqrt{\rho^{2}+(\eta+1)^{2}}-\sqrt{\rho^{2}+(\eta-1)^{2}}}{2 \sqrt{\left(b_{\nu}+b_{\varphi} \eta\right)^{2}+b_{\varphi}^{2} \rho^{2}}} .
$$

Identifying $\varphi=\varphi_{1}, \nu=\varphi_{2}$ (so that $b_{\varphi}=b_{1}, b_{\nu}=b_{2}$ ), and introducing the change of coordinates

$$
\rho=\frac{4 r_{1} r_{2}}{\left(1+r_{1}^{2}+r_{2}^{2}\right)^{2}-4 r_{1}^{2}}, \quad \eta=\frac{\left(1+r_{1}^{2}+r_{2}^{2}\right)\left(1-r_{1}^{2}-r_{2}^{2}\right)}{\left(1+r_{1}^{2}+r_{2}^{2}\right)^{2}-4 r_{1}^{2}},
$$

the general toric metric takes the form

$$
\mathrm{d} s_{\mathrm{EAdS}_{4}}^{2}=\frac{4}{\left(1-r_{1}^{2}-r_{2}^{2}\right)^{2}}\left(\mathrm{~d} r_{1}^{2}+r_{1}^{2} \mathrm{~d} \varphi_{1}^{2}+\mathrm{d} r_{2}^{2}+r_{2}^{2} \mathrm{~d} \varphi_{2}^{2}\right)
$$

which is manifestly the metric of Euclidean $\mathrm{AdS}_{4}$, realised as a hyperbolic ball. In particular, $r_{1}, r_{2} \geq 0$ are constrained by $r_{1}^{2}+r_{2}^{2}<1$, with $\left\{r_{1}^{2}+r_{2}^{2}=1\right\}$ being the conformal boundary $S^{3}$. In these coordinates the conformal factor reads

$$
y\left(r_{1}, r_{2}\right)=\frac{1-r_{1}^{2}-r_{2}^{2}}{\sqrt{2\left(b_{2}^{2}-b_{1}^{2}\right)\left(r_{2}^{2}-r_{1}^{2}\right)+\left(b_{2}-b_{1}\right)^{2}\left(r_{1}^{2}+r_{2}^{2}\right)^{2}+\left(b_{1}+b_{2}\right)^{2}}} .
$$

It is instructive to analyse how the $(\rho, \eta)$ coordinates behave globally, in this simple example. First note that the polar axes map precisely to $\rho=0$. That is, $\rho=0$ if and only 
if $r_{1}=0$ or $r_{2}=0$. Looking more closely at the axes, we have

$$
\begin{aligned}
& \rho\left(r_{1}, 0\right)=0, \quad \eta\left(r_{1}, 0\right)=\frac{1+r_{1}^{2}}{1-r_{1}^{2}}, \\
& \rho\left(0, r_{2}\right)=0, \quad \eta\left(0, r_{2}\right)=\frac{1-r_{2}^{2}}{1+r_{2}^{2}} .
\end{aligned}
$$

In particular the origin $O=\left\{r_{1}=r_{2}=0\right\}$, which is the NUT, maps to the point $(\rho, \eta)=$ $(0,1)$. Indeed, inserting these values into either (C.25) or (C.22), we recover the general expression (3.36) for

$$
y_{\mathrm{NUT}}=\frac{1}{\left|b_{1}+b_{2}\right|} .
$$

The axis $\left(r_{1}, r_{2}\right)=\left(0, r_{2}\right)$ for $r_{2} \in[0,1)$ then maps to $\eta \in(0,1]$, while the axis $\left(r_{1}, r_{2}\right)=$ $\left(r_{1}, 0\right)$ for $r_{1} \in[0,1)$ maps to $\eta \in[1, \infty)$. Notice

$$
\left(1+r_{1}^{2}+r_{2}^{2}\right)^{2}-4 r_{1}^{2} \geq\left(1-r_{1}^{2}\right)^{2} \geq 0
$$

with equality holding in the first inequality if and only if $r_{2}=0$. It follows from this that $\rho \geq 0$ and $\eta>0$. The coordinate region $\left\{r_{1}^{2}+r_{2}^{2}<1\right\}$ then in fact maps one-to-one to the positive quadrant $\{\rho \geq 0, \eta>0\}$, with the axes mapping to $\rho=0$ in the above way. The conformal boundary $\left\{r_{1}^{2}+r_{2}^{2}=1\right\}=\left\{y^{\text {can }}=0\right\}$ is mapped to $\{\eta=0\} \cup\{(\rho=\infty, \eta=\infty)\}$, namely the axis $\rho \geq 0$ plus the point at infinity in $\mathcal{H}^{2}$, the latter corresponding to the point $\left(r_{1}, r_{2}\right)=(1,0)$.

Looking at the collapsing Killing vectors for this solution, we see that $\partial_{\varphi_{2}}$ collapses along $\left\{\rho=0, \eta \geq \lambda_{1}=1\right\}$, while $\partial_{\varphi_{1}}$ collapses along $\{\rho=0, \eta \in(0,1]\}$, where the $(\rho, \eta)$-plane is cut off at $\eta=0$ by the conformal boundary. In fact on the whole of $\mathcal{H}^{2}$ we have that $\partial_{\varphi_{2}}$ collapses on $\left\{\rho=0, \eta \geq \lambda_{1}=1\right\} \cup\left\{\rho=0, \eta \leq \lambda_{2}=-1\right\}$, while $\partial_{\varphi_{1}}$ collapses on the interval $\left\{\rho=0, \eta \in\left[\lambda_{2}, \lambda_{1}\right]=[-1,1]\right\}$. We thus see the division of the $\eta$-axis into the three segments $\lambda_{3}=-\infty<\lambda_{2}=-1<\lambda_{1}=1<\lambda_{0}=\infty$, with different Killing vectors collapsing in each of the 3 regions. However, the conformal boundary actually cuts off half this axis.

It is straightforward to check that the metric in the $(\rho, \eta)$ coordinates is non-singular near the loci where the Killing vectors collapse. Using the expansions, the angular part of the metric reads

$$
\mathrm{d} s_{\text {angular }}^{2}= \begin{cases}\mathrm{d} \varphi^{2}+\frac{\rho^{2}}{\left(\eta^{2}-1\right)^{2}}\left(\mathrm{~d} \nu^{2}-\mathrm{d} \varphi^{2}\right)+\mathcal{O}\left(\rho^{4}\right), & |\eta|>1 \\ \mathrm{~d} \nu^{2}+\frac{\rho^{2}}{\left(1-\eta^{2}\right)^{2}}\left(\mathrm{~d} \varphi^{2}-\mathrm{d} \nu^{2}\right)+\mathcal{O}\left(\rho^{4}\right), & |\eta|<1\end{cases}
$$

and one sees explicitly that $\partial_{\varphi_{1}}=\partial_{\varphi}$ collapses along $\{\rho=0,|\eta|<1\}$, while $\partial_{\varphi_{2}}=\partial_{\nu}$ collapses along $\{\rho=0,|\eta|>1\}$, which agrees with the statements above. The factor in front of this angular part of the metric in (C.1) is

$$
\Upsilon(\rho, \eta)^{2} \equiv \frac{4}{\mathcal{F}^{2}\left[4 \rho^{2}\left(\mathcal{F}_{\rho}^{2}+\mathcal{F}_{\eta}^{2}\right)-\mathcal{F}^{2}\right]}=\frac{4 \sqrt{\rho^{2}+(1-\eta)^{2}} \sqrt{\rho^{2}+(1+\eta)^{2}}}{\left(\sqrt{\rho^{2}+(1-\eta)^{2}}-\sqrt{\rho^{2}+(1+\eta)^{2}}\right)^{2}} .
$$




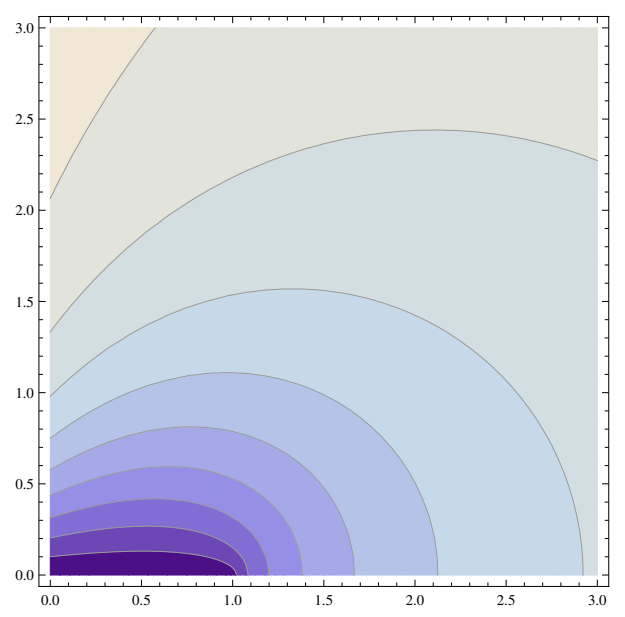

(a) Constant $r_{1}$ contours in the $(\rho, \eta)$ quadrant. The axis $r_{1}=0$ maps to $\eta \in$ $(0,1]$ on $\rho=0$.

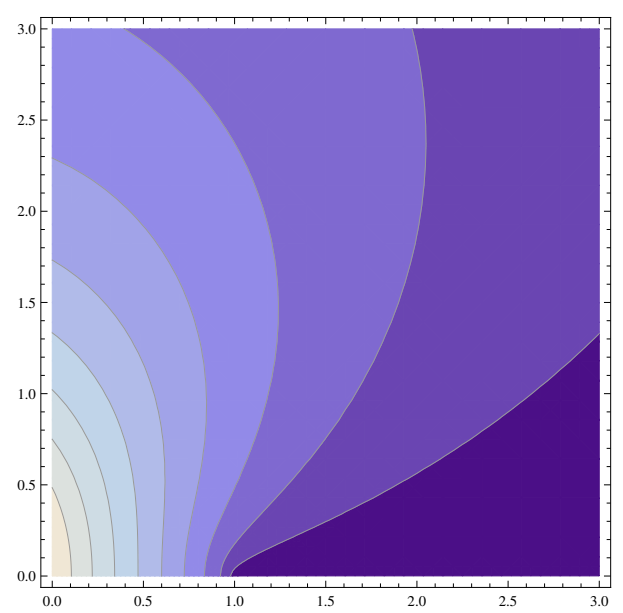

(b) Constant $r_{2}$ contours in the $(\rho, \eta)$ quadrant. The axis $r_{2}=0$ maps to $\eta \in$ $[1, \infty)$ on $\rho=0$.

Figure 2. Contour plots in the $(\rho, \eta)$ quadrant.

Notice that the denominator is non-zero on the quadrant $\{\rho \geq 0, \eta>0\} \subset \mathcal{H}^{2}$, while the numerator is zero precisely at the origin $\rho=0, \eta=1$. This is necessary in order that the metric is regular at the origin. In particular, we find

$$
\left(1-r_{1}^{2}-r_{2}^{2}\right)^{2} \Upsilon^{2} \mathrm{~d} s_{\text {angular }}^{2}=4 r_{1}^{2} \mathrm{~d} \varphi^{2}+4 r_{2}^{2} \mathrm{~d} \nu^{2}+\mathcal{O}\left(r_{1}^{4}, r_{2}^{4}\right) .
$$

Notice that the canonically defined Killing vector $\partial_{\nu}=\partial_{\varphi_{2}}$ has a fixed point set along the axis $r_{2}=0$, which is a copy of $\mathbb{R}^{2}$. Thus the induced Killing vector on the conformal boundary is not a Reeb vector field. Indeed, setting $b_{1}=0, b_{2}=1$ in (C.25) we see that

$$
y^{\operatorname{can}}\left(r_{1}, r_{2}\right)=\frac{1-r_{1}^{2}-r_{2}^{2}}{\sqrt{\left(1+r_{1}^{2}+r_{2}^{2}\right)^{2}-4 r_{1}^{2}}} .
$$

In particular, $y^{\text {can }}\left(r_{1}, 0\right) \equiv 1$ for $r_{1} \in[0,1)$ is constant along the axis where the associated Killing vector $\left\|\partial_{\nu}\right\|=0$. But also $y^{\text {can }}=0$ defines the conformal boundary, which contains the point $\left(r_{1}, r_{2}\right)=(1,0)$. Thus actually $y^{\text {can }}$ is not even a continuous function on the conformal compactification: it is identically 1 along the axis, which intersects the conformal boundary at infinity, where it jumps to 0 . Thus the general expansions we have made are not valid and this case is not covered by our analysis. On the other hand, assuming $b_{1} \neq 0$, $b_{2} \neq 0$ and expanding (C.25) near the points $r_{1}=0, r_{2}=1$ and $r_{1}=1, r_{2}=0$ we find

$$
\begin{aligned}
& y\left(r_{1}=0, r_{2}\right)=\frac{\left(r_{2}-1\right)^{2}}{b_{2}^{2}}+\mathcal{O}\left(\left(r_{2}-1\right)^{3}\right), \\
& y\left(r_{1}, r_{2}=0\right)=\frac{\left(r_{1}-1\right)^{2}}{b_{1}^{2}}+\mathcal{O}\left(\left(r_{1}-1\right)^{3}\right),
\end{aligned}
$$

respectively, so that $y\left(r_{1}, r_{2}\right)$ is now a continuous function on the conformal compactification, and we automatically obtain a non-singular instanton. 
Doing a further change of coordinates, setting

$$
r_{1}=\frac{\sqrt{q^{2}+1}-1}{q} \cos \vartheta, \quad r_{2}=\frac{\sqrt{2+q^{2}-2 \sqrt{1+q^{2}}} \sin \vartheta}{q},
$$

the metric becomes

$$
\mathrm{d} s_{\mathrm{EAdS}_{4}}^{2}=\frac{\mathrm{d} q^{2}}{q^{2}+1}+q^{2}\left(\mathrm{~d} \vartheta^{2}+\cos ^{2} \vartheta \mathrm{d} \varphi_{1}^{2}+\sin ^{2} \vartheta \mathrm{d} \varphi_{2}\right)
$$

and the conformal factor reads

$$
y(q, \vartheta)=\frac{1}{\sqrt{\left(b_{2}+b_{1} \sqrt{q^{2}+1}\right)^{2} \cos ^{2} \vartheta+\left(b_{1}+b_{2} \sqrt{q^{2}+1}\right)^{2} \sin ^{2} \vartheta}}
$$

in agreement with the formulas in section 5.1.

Finally, let us present the instanton in the various coordinate systems introduced. In the original $(\rho, \eta)$ coordinates the general instanton simplifies to

$$
\begin{aligned}
F= & \frac{y(\rho, \eta)\left(b_{1}^{2}-b_{2}^{2}\right) \sqrt{\rho} \mathcal{F}_{\mathrm{EAdS}}(\rho, \eta)}{2 c_{11} \sqrt{\left(\rho^{2}+(\eta+1)^{2}\right)\left(\rho^{2}+(\eta-1)^{2}\right)}}\left[\left(g^{13}+g^{24}\right)\left(\hat{\eta}\left(\eta^{2}-1\right)-\rho^{2}\left(b_{2}+3 b_{1} \eta\right)\right)\right. \\
& \left.+\rho\left(g^{12}-g^{34}\right)\left(2 b_{2} \eta+b_{1}\left(3 \eta^{2}-1\right)-b_{1} \rho^{2}\right)\right] .
\end{aligned}
$$

In the $\left(r_{1}, r_{2}\right)$-coordinates, we have instead

$$
\begin{aligned}
F= & \frac{2\left(b_{2}^{2}-b_{1}^{2}\right) y\left(r_{1}, r_{2}\right)^{3}}{\left(1-r_{1}^{2}-r_{2}^{2}\right)^{3}}\left[( r _ { 1 } \mathrm { d } r _ { 1 } \wedge \mathrm { d } \varphi _ { 1 } - r _ { 2 } \mathrm { d } r _ { 2 } \mathrm { d } \varphi _ { 2 } ) \left(b_{2}\left(1-r_{1}^{2}+r_{2}^{2}\right)\right.\right. \\
& \left.\left.+b_{1}\left(1+r_{1}^{2}-r_{2}^{2}\right)\right)-2\left(b_{2}-b_{1}\right) r_{1} r_{2}\left(r_{2} \mathrm{~d} r_{1} \wedge \mathrm{d} \varphi_{2}+r_{1} \mathrm{~d} r_{2} \wedge \mathrm{d} \varphi_{1}\right)\right],
\end{aligned}
$$

and the corresponding gauge field reads

$$
A=\frac{\left[b_{1}\left(1-r_{1}^{2}-r_{2}^{2}\right)+b_{2}\left(1+r_{1}^{2}+r_{2}^{2}\right)\right] \mathrm{d} \varphi_{1}+\left[b_{1}\left(1+r_{1}^{2}+r_{2}^{2}\right)+b_{2}\left(1-r_{1}^{2}-r_{2}^{2}\right)\right] \mathrm{d} \varphi_{2}}{2 \sqrt{2\left(b_{2}^{2}-b_{1}^{2}\right)\left(r_{2}^{2}-r_{1}^{2}\right)+\left(b_{1}-b_{2}\right)^{2}\left(r_{1}^{2}+r_{2}^{2}\right)^{2}+\left(b_{1}+b_{2}\right)^{2}}} .
$$

In the $(\vartheta, q)$ coordinates this becomes

$$
A=\frac{\left(b_{1}+b_{2} \sqrt{q^{2}+1}\right) \mathrm{d} \varphi_{1}+\left(b_{2}+b_{1} \sqrt{q^{2}+1}\right) \mathrm{d} \varphi_{2}}{2 \sqrt{\left(b_{2}+b_{1} \sqrt{q^{2}+1}\right)^{2} \cos ^{2} \vartheta+\left(b_{1}+b_{2} \sqrt{q^{2}+1}\right)^{2} \sin ^{2} \vartheta}}
$$

which is the expression written in section 5.1 and originally presented in [9].

\section{C.4 Plebanski-Demianski from 3-pole solution}

In section 5.3 we discussed a two-parameter family of self-dual Einstein metrics on the fourball. Although this was constructed in [10] starting from the local Plebanski-Demianski metric, it turns out that it is related to the 3-pole solutions of section C.2. However, the relationship is complicated and involves various changes of coordinates and conformal 


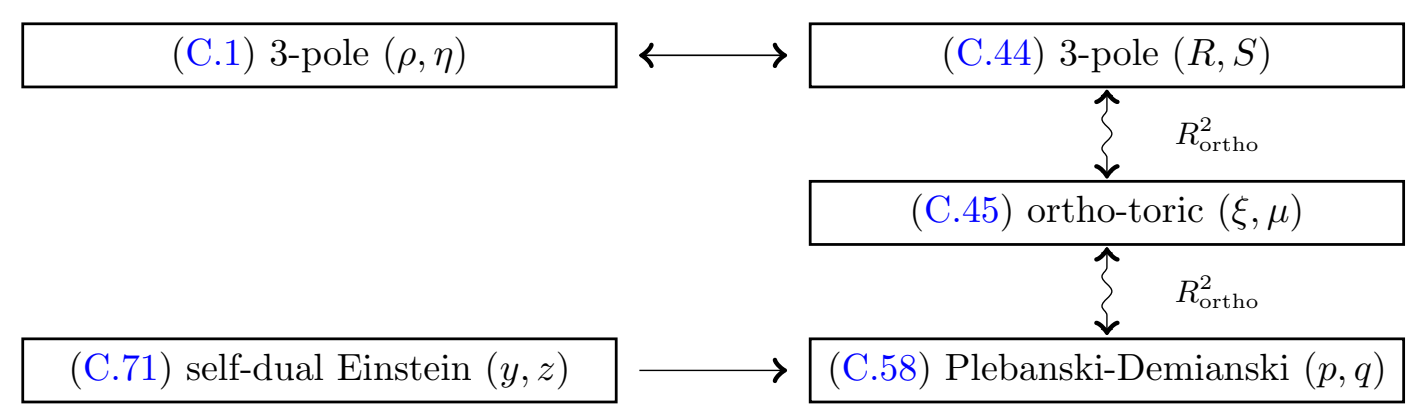

Figure 3. Overview of the metrics and coordinate transformations in this subsection. Straight arrows denote changes of coordinates, while wavy arrows denote conformal transformations (with the indicated conformal factor). The bottom arrow points only one way, to represent the fact that the Plebanski-Demianski metric, with a choice of Killing vector $K$, is a special case of the general self-dual Einstein metric.

transformations. These are illustrated in figure 3, and in the rest of this section will discuss the links in detail. Some of these relations were discussed in [26], albeit somewhat implicitly. In this section we will not be interested in global properties of these metrics, as metrics on the ball; in particular the various angular coordinates that will be introduced do not have canonical periodicities, and the action of the associated Killing vectors generically have non-closed orbits. Global properties were discussed in detail in [10], in the $(p, q)$ coordinate system.

From $(\rho, \eta)$ coordinates to $(\boldsymbol{R}, S)$ coordinates. We begin noting that applying the $\operatorname{PSL}(2, \mathbb{R})$ symmetry transformation in (C.19), (C.20) to the $m=3$ case, we see that we can choose for example $\lambda_{1}^{\prime}=1, \lambda_{2}^{\prime}=0, \lambda_{3}^{\prime}=-1$, so that the general 3 -pole solution can be written in the form

$$
\mathcal{F}(\rho, \eta)=\frac{b+c}{2} \frac{\sqrt{\rho^{2}+(\eta+1)^{2}}}{\sqrt{\rho}}+\frac{a}{\sqrt{\rho}}+\frac{b-c}{2} \frac{\sqrt{\rho^{2}+(\eta-1)^{2}}}{\sqrt{\rho}},
$$

as presented in [26]. ${ }^{26}$ Using the residual scaling symmetry one of the three parameters $a, b, c$ could be set to an arbitrary non-zero value. However, in the following we will find it convenient to keep the three parameters in the expressions. The metric (C.1) with $\mathcal{F}(\rho, \eta)$ given by (C.40) corresponds to the top left corner of figure 3. Following [26], let us define new coordinates ${ }^{27} R, \hat{\theta}$ as

$$
\rho=\sqrt{R^{2}-1} \cos \hat{\theta}, \quad \eta=R \sin \hat{\theta},
$$

so that

$$
y^{\mathrm{can}}(R, \theta)=a+b R+c \sin \hat{\theta},
$$

\footnotetext{
${ }^{26}$ In [26] the 3-pole solution appears written in terms of a parameter $m$, such that $m^{2}=\mp 1$. In this reference these two cases are referred to as Type I and Type II metrics, respectively. Here we are interested only in the case $m^{2}=1$, corresponding to the expression in (C.40).

${ }^{27}$ This change of coordinates may be easily inverted as

$$
R^{2}=\frac{1}{2}\left(1+\rho^{2}+\eta^{2}+\sqrt{\left(1+\rho^{2}+\eta^{2}\right)^{2}-4 \eta^{2}}\right), \quad \sin \hat{\theta}=\frac{\eta}{R} .
$$
}



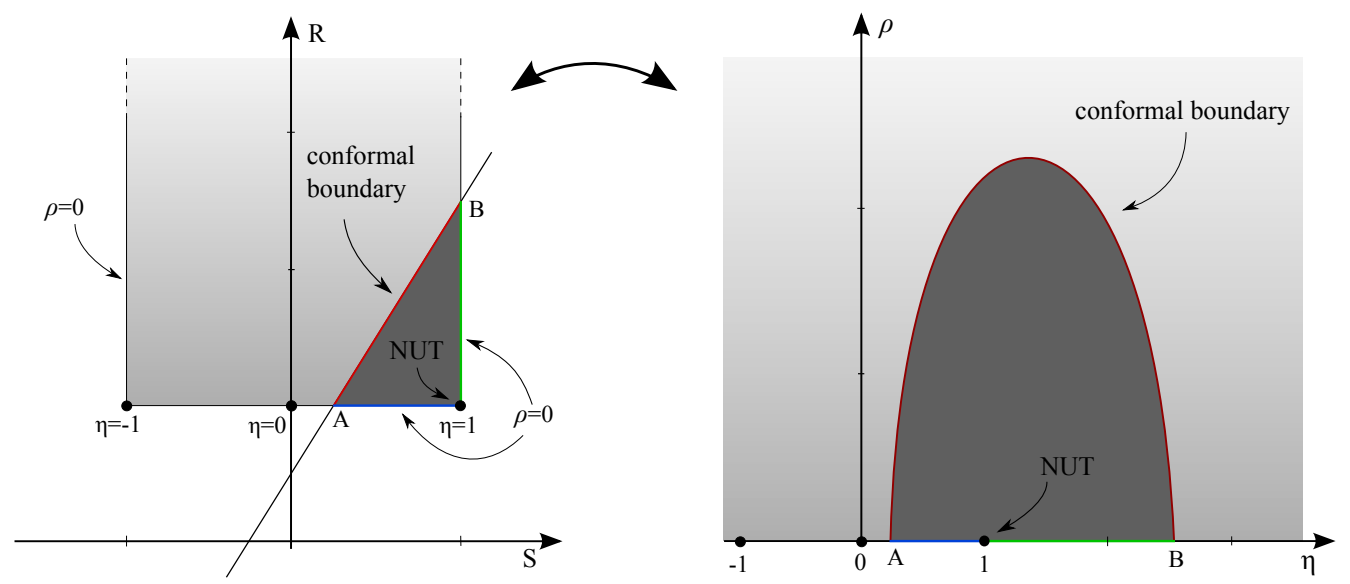

Figure 4. An illustration of the map between $(\rho, \eta)$-coordinates and $(R, S)$-coordinates: $\rho=$ $\sqrt{R^{2}-1} \sqrt{1-S^{2}}, \eta=R S$. The conformal boundary, defined by $y^{\text {can }}=0$, is simply a segment in the $(R, S)$ plane. This is mapped to an arc intersecting the $\rho=0$ axis at two points ( $A$ and $B$ ). The three marked points on this axis $\eta=-1,0,1$ correspond to the location of the three monopoles in (C.40), with $\eta=1$ corresponding to the NUT. The parameters $a, b, c$ are choosen to correspond to region $C$ in figure 3 of [26].

and

$$
\rho^{-1}\left(\frac{1}{4} \mathcal{F}^{2}-\rho^{2}\left(\mathcal{F}_{\rho}^{2}+\mathcal{F}_{\eta}^{2}\right)\right)=\frac{b(a R+b)-c(a \sin \hat{\theta}+c)}{R^{2}-\sin ^{2} \hat{\theta}}
$$

Then further defining $S=\sin \hat{\theta}$, the metric (C.1) becomes ${ }^{28}$

$$
\begin{aligned}
& \mathrm{d} s_{R S}^{2}=\frac{c^{2}-b^{2}-a(b R-c S)}{(a+b R+c S)^{2}}\left(\frac{\mathrm{d} R^{2}}{R^{2}-1}+\frac{\mathrm{d} S^{2}}{1-S^{2}}\right) \\
& \quad+\frac{1}{(a+b R+c S)^{2}\left(c^{2}-b^{2}-a(b R-c S)\right)\left(R^{2}-S^{2}\right)} \\
& \quad \times\left(\left(R^{2}-1\right)\left(1-S^{2}\right)[(b R-c S) \mathrm{d} \nu+(b S-c R) \mathrm{d} \varphi]^{2}+\left[\left(b S\left(R^{2}-1\right)+c R\left(1-S^{2}\right)\right) \mathrm{d} \nu\right.\right. \\
& \left.\left.\quad-\left(c S\left(R^{2}-1\right)+b R\left(1-S^{2}\right)+a\left(R^{2}-S^{2}\right)\right) \mathrm{d} \varphi\right]^{2}\right) .
\end{aligned}
$$

This form of the 3-pole metric appears at page 21 of [26] (with opposite sign — see footnote 25) and in figure 3 it corresponds to the second box in the upper part. Reference [26] discusses the moduli space of these metrics, parametrized by $(a, b, c)$, including different topologies and boundary conditions. Here we are only interested in the negative curvature case, and we note that in general the domain of existence in the $(R, S)$ plane is strictly contained in the strip $R \in[1,+\infty), S \in[-1,1]$, where the conformal boundary is a segment on the line $a+b R+c S=0$. In figure 4 this domain is the triangle on the right-bottom

\footnotetext{
${ }^{28}$ We find that the formula corresponding to (C.43) in [26] (middle of page 18) has a sign error. Moreover, the angular variables $\psi, \varphi$ in the metric $g_{R S}$ in [26] (top of page 21) are inverted with respect to those in our equation (C.44).
} 
corner of the strip, which maps back to a compact domain in the $(\rho, \eta)$ plane. Although this behaviour appears to be qualitatively different from that in the $\mathrm{AdS}_{4} / 2$-pole case of the previous section, we notice that via $\operatorname{PSL}(2, \mathbb{R})$ transformations we may first shift the point $B$ in figure 4 to the origin, and then using inversion we may map this to $\infty$. In this way $y^{\text {can }}=0$ becomes a semi-infinite line joining a point $\left(\rho=0, \eta_{0}\right)$ on the $\rho=0$ axis to $\infty$, as in the $\mathrm{AdS}_{4} / 2$-pole case. However, we will not further discuss global issues of the 3 -pole metrics in the $(\rho, \eta)$ coordinates, referring to [10] for global considerations, in the final $(p, q)$ (Plebanski-Demianski) coordinates.

From $(\boldsymbol{R}, \boldsymbol{S})$ coordinates to $(\boldsymbol{\xi}, \boldsymbol{\mu})$ coordinates. It was noted in [26] that the 3-pole metric must be conformally related to the following ortho-toric [52] Kähler metric

$$
\begin{aligned}
\mathrm{d} s_{\text {ortho }}^{2}= & (\xi-\mu)\left(\frac{\mathrm{d} \xi^{2}}{f(\xi)}-\frac{\mathrm{d} \mu^{2}}{f(\mu)}\right)+\frac{1}{\xi-\mu}\left[f(\xi)(\mathrm{d} t+\mu \mathrm{d} v)^{2}\right. \\
& \left.-f(\mu)(\mathrm{d} t+\xi \mathrm{d} v)^{2}\right]
\end{aligned}
$$

with

$$
f(x)=\left(x-x_{1}\right)\left(x-x_{2}\right)\left(x-x_{3}\right)\left(x-x_{4}\right),
$$

and $x_{1}+x_{2}+x_{3}+x_{4}=0$, so that the quartic polynomial has no cubic term. Here $\xi+\mu$ and $\xi \mu$ are the trace and the Pfaffian of the normalized Ricci form of the metric (C.44), and $(\xi+\mu)^{2}$ is the conformal factor necessary to pass from the Einstein metric (C.44) to the Kähler metric (C.45). Note that this Kähler metric is not scalar flat, and therefore it cannot be related by a change of coordinates to the canonical Kähler metric of section 2.2. Indeed, it will become clear shortly that one has to make two different conformal transformations to relate the Kähler metric (C.45) to the Kähler metric (2.9).

The conformal transformation relating (C.44) and (C.45) reads

$$
\mathrm{d} s_{\text {ortho }}^{2}=\kappa^{2}\left(\frac{a+b R+c S}{b^{2}-c^{2}+a(b R-c S)}\right)^{2} \mathrm{~d} s_{R S}^{2},
$$

where $\kappa$ is an arbitrary constant. In particular, computing the Ricci scalars of the metrics on each side of equation (C.47) and equating these, yields the relation

$$
\frac{\kappa^{3}}{a\left(b^{2}-c^{2}\right)}(\xi+\mu)=\kappa \frac{a+b R+c S}{b^{2}-c^{2}+a(b R-c S)} .
$$

As noted below (C.40), using the scaling symmetry the three parameters $a, b, c$ can be multiplied by an overall non-zero constant, while $g_{R S}$ is invariant. For example one could arrange for the conformal factor (C.47) to be simply $(\xi+\mu)^{2}$. Instead, leaving the arbitrary paramater $\kappa$, we find that the change of coordinates is given by

$$
\begin{aligned}
R=\frac{1}{2 a b \Delta}[ & 2 \kappa^{2}\left(\left(b^{2}-c^{2}\right)^{2}-3 a^{2} b^{2}-a^{2} c^{2}\right)(\xi+\mu)+4 \kappa^{4}\left(a^{2}+b^{2}-c^{2}\right) \xi \mu \\
& \left.+\left(b^{2}-c^{2}\right)^{3}+a^{2}\left(4 a^{2} b^{2}-3 b^{4}+c^{4}+2 b^{2} c^{2}\right)\right], \\
S=\frac{1}{2 a c \Delta}[ & 2 \kappa^{2}\left(\left(b^{2}-c^{2}\right)^{2}-3 a^{2} c^{2}-a^{2} b^{2}\right)(\xi+\mu)+4 \kappa^{4}\left(a^{2}-b^{2}+c^{2}\right) \xi \mu \\
- & \left.\left(b^{2}-c^{2}\right)^{3}+a^{2}\left(4 a^{2} c^{2}-3 c^{4}+b^{4}+2 b^{2} c^{2}\right)\right],
\end{aligned}
$$


where

$$
\Delta \equiv 2 \kappa^{2} a^{2}(\xi+\mu)-4 \kappa^{4} \xi \mu-2 a^{2}\left(b^{2}+c^{2}\right)+\left(b^{2}-c^{2}\right)^{2} .
$$

Note that $R$ and $S$ are rational functions of the trace $\xi+\mu$ and the Pfaffian $\xi \mu$. The polynomial $f(x)$ takes the form

$f(x)=\left(x-\frac{-b^{2}+c^{2}-2 a b}{2 \kappa^{2}}\right)\left(x-\frac{-b^{2}+c^{2}+2 a b}{2 \kappa^{2}}\right)\left(x-\frac{b^{2}-c^{2}-2 a c}{2 \kappa^{2}}\right)\left(x-\frac{b^{2}-c^{2}+2 a c}{2 \kappa^{2}}\right)$.

The angular coordinates are linearly related as

$$
\begin{aligned}
& \varphi=\varepsilon \frac{a b c\left(c^{2}-b^{2}\right)}{\kappa^{6}}\left(2 \kappa^{2} t+a^{2} v\right), \\
& \nu=\varepsilon \frac{a\left(b^{2}-c^{2}\right)}{2 \kappa^{6}}\left(2 \kappa^{2}\left(a^{2}-b^{2}-c^{2}\right) t+\left(a^{2}\left(b^{2}+c^{2}\right)-\left(b^{2}-c^{2}\right)^{2}\right) v\right),
\end{aligned}
$$

with the choices $\varepsilon= \pm 1$. Setting $\kappa^{2}=\frac{1}{2}$ by using the scaling symmetry, one recovers the form of $f(x)$ written in [26], up to a change of sign of the roots $x_{i} \rightarrow-x_{i}$, due to the different overall sign difference in the metrics $\mathrm{d} s_{R S}^{2}$ here and in [26].

The inverse change of coordinates is given by

$$
\begin{aligned}
& \xi=\frac{1}{2 \kappa^{2}} \frac{a\left(b^{2}-c^{2}\right)(a+b R+c S) \pm \sqrt{W(R, S)}}{b^{2}-c^{2}+a(b R-c S)} \\
& \mu=\frac{1}{2 \kappa^{2}} \frac{a\left(b^{2}-c^{2}\right)(a+b R+c S) \mp \sqrt{W(R, S)}}{b^{2}-c^{2}+a(b R-c S)}
\end{aligned}
$$

where

$$
\begin{aligned}
W(R, S) \equiv & 4 a^{4} b^{2} c^{2}\left(R^{2}+S^{2}\right)+4 a^{2} b c\left(\left(b^{2}-c^{2}\right)^{2}-a^{2}\left(c^{2}+b^{2}\right)\right) R S \\
& +\left(b^{2}-c^{2}\right)^{2}(a-b-c)(a+b-c)(a-b+c)(a+b+c) .
\end{aligned}
$$

Let us show that this makes sense, checking that $W(R, S) \geq 0$ everywhere in the domain of existence of the metric. Of course, it is sufficient to show that $W(R, S) \geq 0$ in the strip $[1,+\infty) \times[-1,1]$. By explicit computation one finds that for any value of $(R, S)$ the unique solution of $\partial_{R} W=\partial_{S} W=0$ is $R=S=0$, so that $W$ does not have an extremal point in the interior of $[1,+\infty) \times[-1,1]$. On the boundary of this strip we compute

$$
\begin{gathered}
W(R, S= \pm 1)=\left(\left(b^{2}-c^{2}\right)^{2}-a^{2}\left(b^{2}+c^{2} \mp 2 b c R\right)\right)^{2}, \\
W(R=1, S)=\left(\left(b^{2}-c^{2}\right)^{2}-a^{2}\left(b^{2}+c^{2}-2 b c S\right)\right)^{2},
\end{gathered}
$$

and for $R \rightarrow+\infty$ we have $W \rightarrow 4 a^{4} b^{2} c^{2} R^{2}>0$ for any $S \in[-1,1]$, therefore for any large value $R_{c}$ of $R, W$ is non-negative. Since $W(R, S)$ is a continuous bounded function, by the extreme value theorem it must attain an absolute maximum and an absolute minimum on the boundary of the domain $\left[1, R_{c}\right] \times[-1,1]$. As there are no extremal points in the interior of the domain, it follows that the maximum and the minimum must be on the boundary. Therefore, since at the boundary $W \geq 0$, the absolute minimum is also non-negative. This is clearly still true when we let the cut-off $R_{c} \rightarrow \infty$. 
Notice that the ortho-toric Kähler metric (C.45) strongly resembles the PlebanskiDemianski Einstein metric (5.28). In particular, both metrics are characterized by a quartic polynomial without cubic term. Thus it would be tempting to think that, up to a conformal transformation, the two sets of coordinates may be simply related. However, this is not the case.

From $(\xi, \mu)$ coordinates to $(\boldsymbol{p}, \boldsymbol{q})$ coordinates. Here we will show that after undoing the conformal transformation (C.47), transforming the ortho-toric Kähler metric (C.45) into an Einstein self-dual metric, this is related by a non-trivial change of coordinates to the Plebanski-Demianski metric (5.28). In particular, we will show that

$$
\mathrm{d} s_{\mathrm{PD}}^{2}=\frac{8 a^{2}\left(b^{2}-c^{2}\right)^{2}}{(\xi+\mu)^{2}} \mathrm{~d} s_{\text {ortho }}^{2},
$$

displaying the explicit change of coordinates. Note that the conformal factor is simply proportional to the square of the Ricci scalar of the ortho-toric Kähler metric, namely $R_{\text {ortho }}=-12(\xi+\mu)$.

Recall the Plebanski-Demianski metric in the $(p, q)$-coordinates reads

$$
\mathrm{d} s_{\mathrm{PD}}^{2}=\frac{\mathcal{P}(q)}{q^{2}-p^{2}}\left(\mathrm{~d} \tau+p^{2} \mathrm{~d} \sigma\right)^{2}-\frac{\mathcal{P}(p)}{q^{2}-p^{2}}\left(\mathrm{~d} \tau+q^{2} \mathrm{~d} \sigma\right)^{2}+\frac{q^{2}-p^{2}}{\mathcal{P}(q)} \mathrm{d} q^{2}-\frac{q^{2}-p^{2}}{\mathcal{P}(p)} \mathrm{d} p^{2},
$$

where

$$
\begin{aligned}
\mathcal{P}(x) & =\left(x-p_{1}\right)\left(x-p_{2}\right)\left(x-p_{3}\right)\left(x-p_{4}\right) \\
& \equiv x^{4}+E x^{2}-2 M x+L .
\end{aligned}
$$

Here we will denote the constant coefficient of the quartic $\mathcal{P}(x)$ with the symbol $L$, instead of using the notation " $-Q^{2}+\alpha$ " of [10]. This is to emphasize the fact that $L$ is a genuine metric parameter, while $Q$ and $\alpha$ are not, and are meaningful only when discussing the instanton. Rewriting the polynamial $f(x)$ in (C.52) as

$$
f(x)=x^{4}+H x^{2}+T x+U,
$$

where $T=8 a^{2}\left(b^{2}-c^{2}\right)^{2}$, the non-angular part of the change of coordinates is then given by

$$
\begin{aligned}
& \xi=\left(\frac{T}{4 M^{2}}\right)^{1 / 3} \frac{-M \pm \frac{1}{2} \sqrt{\Xi(p, q)}}{(p+q)}, \\
& \mu=\left(\frac{T}{4 M^{2}}\right)^{1 / 3} \frac{-M \mp \frac{1}{2} \sqrt{\Xi(p, q)}}{(p+q)},
\end{aligned}
$$

with

$$
\Xi(p, q)=4 M^{2}+4 M(p+q)(2 p q-E)+(p+q)^{2}\left(E^{2}-4 L\right) .
$$

Let us postpone showing that $\Xi(p, q) \geq 0$ until the end of this subsection. Remarkably, in spite of this complicated relationship, in both sets of coordinates the metrics are characterized by a single quartic polynomial without the cubic term. The angular coordinates 
are given by the linear combination,

$$
t=\left(\frac{|M|}{4 T^{2}}\right)^{1 / 3}(E \sigma-2 \tau), \quad v=\frac{2|M|}{T} \sigma
$$

while the remaining parameters are related as

$$
\begin{aligned}
& H=-\frac{1}{4}\left(\frac{T^{2}}{2 M^{4}}\right)^{1 / 3}\left(E^{2}-4 L\right), \\
& U=\frac{1}{64}\left(\frac{T^{2}}{2 M^{4}}\right)^{2 / 3}\left(32 M^{2} E+\left(E^{2}-4 L\right)^{2}\right) .
\end{aligned}
$$

Of course these parameters are also related to the original constants $a, b, c$ as

$$
\begin{aligned}
& H=-2\left(\left(b^{2}-c^{2}\right)^{2}+2 a^{2}\left(b^{2}+c^{2}\right)\right), \\
& U=16 a^{4} b^{2} c^{2}+\left(b^{2}-c^{2}\right)^{4}-4 a^{2}\left(b^{2}-c^{2}\right)^{2}\left(b^{2}+c^{2}\right) .
\end{aligned}
$$

Let us now show that $\Xi(p, q) \geq 0$ in the domain of definition of the coordinates $(p, q)$. Adopting the conventions of [10], we have $p \in\left[p_{3}, p_{4}\right]$ and $q \in\left[p_{4},+\infty\right)$, with $p_{4}>0$ and $p_{4}>p_{3}$, and we must check that $\Xi(p, q) \geq 0$ everywhere in the strip $\left[p_{3}, p_{4}\right] \times\left[p_{4},+\infty\right]$. On the boundary of the strip, we find ${ }^{29}$

$$
\begin{aligned}
& \Xi\left(p_{3}, q\right)=\left(p_{3}^{3}+p_{1} p_{3} p_{4}+p_{1} p_{4}\left(p_{1}+p_{4}\right)-q\left(p_{1}^{2}+p_{1} p_{4}+p_{4}^{2}-p_{3}^{2}+p_{1} p_{3}+p_{3} p_{4}\right)\right)^{2}, \\
& \Xi\left(p_{4}, q\right)=\left(p_{1}^{2}\left(p_{3}-q\right)+p_{1}\left(p_{3}+p_{4}\right)\left(p_{3}-q\right)-p_{3}^{2} q+p_{4} q\left(p_{4}-p_{3}\right)+p_{4}^{3}\right)^{2}, \\
& \Xi\left(p, p_{4}\right)=\left(-p\left(p_{1}^{2}+p_{1}\left(p_{3}+p_{4}\right)+p_{3}^{2}+p_{3} p_{4}-p_{4}^{2}\right)+p_{1} p_{3} p_{4}+p_{1} p_{3}\left(p_{1}+p_{3}\right)+p_{4}^{3}\right)^{2}
\end{aligned}
$$

and for $q \rightarrow \infty$ we have

$$
\Xi \rightarrow q^{2}\left(8 M p+E^{2}-4 L\right) \equiv q^{2} \Xi_{\infty}(p) .
$$

One checks that $\Xi_{\infty}(p) \geq 0$ at $p=p_{3}$ and $p=p_{4}$, and because $\Xi_{\infty}(p)$ is linear in $p$ one concludes that $\Xi(p, q) \geq 0$ for all $p \in\left[p_{3}, p_{4}\right]$ for $q \rightarrow \infty$. Therefore $\Xi(p, q) \geq 0$ on the boundary of the rectangular domain $\left[p_{3}, p_{4}\right] \times\left[p_{4}, q_{c}\right]$, for any large $q_{c}$. A computation shows that there exist four points $(p, q)$ where $\partial_{p} \Xi=\partial_{q} \Xi=0$. Two of these points are

$$
p=-q= \pm \sqrt{\frac{-E}{2}},
$$

and exist only if $E \leq 0$. In any case, it's easy to see that the line $p=-q$ intersects the strip only at $q=p_{4}, p=p_{3}=-p_{4}$. In [10] it is shown that in order for the instanton to be non-singular, the condition $p_{3}+p_{4}>0$ must hold, so these two points are never inside the strip. Two further points take the form

$$
p=q=\alpha+\sqrt{\beta} \quad \text { and } \quad p=q=\alpha-\sqrt{\beta},
$$

for some combinations of the parameters denoted $\alpha$ and $\beta$, and again it's simple to see that the line $p=q$ intersects the strip only at $p=p_{4}=q$. Therefore there are no extremal points in the interior of the domain $\left[p_{3}, p_{4}\right] \times\left[p_{4}, q_{c}\right]$ and the argument to conclude that $\Xi(p, q) \geq 0$ is then exactly the same as that used earlier to show that $W(R, S) \geq 0$.

\footnotetext{
${ }^{29}$ Here we have used $p_{2}=-p_{1}-p_{3}-p_{4}$.
} 
From $(y, u)$ coordinates to $(p, q)$ coordinates. Finally, let us show that the Plebanski-Demianski metric can be cast in the canonical coordinates characterising the self-dual Einstein metric (2.4), which we recall here

$$
\mathrm{d} s_{\mathrm{SDE}}^{2}=\frac{1}{y^{2}}\left[V^{-1}(\mathrm{~d} \psi+\phi)^{2}+V\left(\mathrm{~d} y^{2}+4 \mathrm{e}^{w} \mathrm{~d} z \mathrm{~d} \bar{z}\right)\right] .
$$

Although in principle this can be done for any choice of Killing vector $K$, the general expressions are unwieldy. We will present an expression for the instanton constructed from a general Killing vector in subsection C.5. Here we will consider only the special choices of Killing vector $K$ corresponding to the instantons studied in [10], for which the formulas simplify considerably. Moreover, as assumed everywhere in this paper, we have to restrict to the real solutions in [10], so that in particular $Q$ and $\sqrt{\alpha}$ are real.

Starting from $[10]$

$$
K=\partial_{\psi}=2 \sqrt{\alpha} \partial_{\tau}+2 \partial_{\sigma}
$$

where

$$
2 \sqrt{\alpha}=\frac{M^{2}}{Q^{2}}+E
$$

and using equations (3.33) and (3.34), in section 5.3 we find that

$$
\frac{1}{y(p, q)^{2}}=\frac{4\left(Q^{2}(p+q)+M p q-M \sqrt{\alpha}\right)^{2}}{Q^{2}} .
$$

Notice that the relation (C.73) was derived in [10] by imposing supersymmetry, but in doing so the authors were employing a specific ansatz for $F$. It is the compatibility of that ansatz with supersymmetry that yielded (C.73). However, in our general context we know that supersymmetry is automatic for any choice of Killing vector, and therefore we cannot expect a new relation, such as (C.73) to be found. Thus (C.73) corresponds merely to a very special choice of Killing vector. This will become more manifest after writing the general instanton in section C.5.

Assuming the second $\mathrm{U}(1)$ isometry of the general metric (C.71) may be parametrized by a local angular coordinate $\Theta$, defined through $z=u \mathrm{e}^{\mathrm{i} \Theta}$, the self-dual Einstein metric becomes

$$
\mathrm{d} s_{\mathrm{SDE}}^{2}=\frac{1}{y^{2}}\left[V^{-1}(\mathrm{~d} \psi+\phi)^{2}+V\left(\mathrm{~d} y^{2}+4 \mathrm{e}^{w}\left(\mathrm{~d} u^{2}+u^{2} \mathrm{~d} \Theta^{2}\right)\right)\right] .
$$

The angular coordinates $(\psi, \Theta)$ must be linearly related to the angular coordinates $(\sigma, \tau)$ of the Plebanski-Demianski metric (C.58) as

$$
\left(\begin{array}{l}
\tau \\
\sigma
\end{array}\right)=\left(\begin{array}{ll}
A & C \\
B & D
\end{array}\right)\left(\begin{array}{l}
\psi \\
\Theta
\end{array}\right)
$$

where $A=2 \sqrt{\alpha}$ and $B=2$, whereas the entries $C, D$ are arbitrary, provided the transformation (C.76) is invertible. Comparing the relevant terms, we find that the function $V(p, q)$ is

$$
V(p, q)=\frac{1}{4 y(p, q)^{2}} \frac{q^{2}-p^{2}}{\mathcal{P}(q)\left(\sqrt{\alpha}+p^{2}\right)^{2}-\mathcal{P}(p)\left(\sqrt{\alpha}+q^{2}\right)^{2}},
$$


and the one-form $\phi$ is

$$
\phi=\frac{1}{2} \frac{\mathcal{P}(q)\left(\sqrt{\alpha}+p^{2}\right)\left(C+D p^{2}\right)-\mathcal{P}(p)\left(\sqrt{\alpha}+q^{2}\right)\left(C+D q^{2}\right)}{\mathcal{P}(q)\left(\sqrt{\alpha}+p^{2}\right)^{2}-\mathcal{P}(p)\left(\sqrt{\alpha}+q^{2}\right)^{2}} \mathrm{~d} \Theta .
$$

The coordinate $u$ is found by integrating the following relation

$$
\frac{\mathrm{d} u^{2}}{u^{2}}=\frac{1}{Q^{2}(C-D \sqrt{\alpha})^{2}}\left(\frac{\left(M p+Q^{2}\right)}{\mathcal{P}(p)} \mathrm{d} p+\frac{\left(M q+Q^{2}\right)}{\mathcal{P}(q)} \mathrm{d} q\right)^{2} .
$$

If all four roots $p_{i}$ of the polynomial $\mathcal{P}(x)$ are distinct this is solved by

$$
u(p, q)=\tilde{C} \prod_{i=1}^{4}\left[\left(p-p_{i}\right)\left(q-p_{i}\right)\right]^{ \pm \frac{\left(M p_{i}+Q^{2}\right)}{Q(C-D \sqrt{\alpha}) \mathcal{P}^{\prime}\left(p_{i}\right)}},
$$

where $\tilde{C}$ an integration constant. When $p_{1}=p_{2}$, equation (C.79) can also be solved by a function $u(p, q)$, but we will not give this expression here. Finally, the function $w(p, q)$ is given by

$$
\mathrm{e}^{w(p, q)}=-\frac{y(p, q)^{4}}{u(p, q)^{2}}(C-D \sqrt{\alpha})^{2} \mathcal{P}(p) \mathcal{P}(q) .
$$

From these expressions, one can verify that equations (2.5), (2.6), and (2.7) are satisfied.

Notice that choosing $C=0$ and $D=\frac{1}{2 \sqrt{\alpha}}$ so that the angular change of variables (C.76) is an $\mathrm{SL}(2, \mathbb{R})$ transformation, the formulas simplify slightly. One can also write more concrete expressions for $u(p, q)$ in (C.80), obtained upon using the various solutions for $Q$ [10]:

$$
Q=\left\{\begin{array}{l} 
\pm \frac{\left(p_{3}+p_{1}\right)\left(p_{4}+p_{1}\right)}{2} \\
\pm \frac{\left(p_{3}+p_{4}\right)\left(p_{3}+p_{1}\right)}{2} \\
\pm \frac{\left(p_{3}+p_{4}\right)\left(p_{4}+p_{1}\right)}{2}
\end{array}\right.
$$

where, as we already noticed, here we restrict to the region of parameter space where $Q$ is everywhere real. We refer to [10] for details. Setting $\tilde{C}=1$, and fixing a choice of sign, in the first case we have

$$
u(p, q)=\left(\frac{\left(p-p_{2}\right)\left(q-p_{2}\right)}{\left(p-p_{1}\right)\left(q-p_{1}\right)}\right)^{\frac{1}{p_{1}-p_{2}}}\left(\frac{\left(p-p_{3}\right)\left(q-p_{3}\right)}{\left(p-p_{4}\right)\left(q-p_{4}\right)}\right)^{\frac{1}{p_{3}-p_{4}}},
$$

in the second case we have

$$
u(p, q)=\left(\frac{\left(p-p_{2}\right)\left(q-p_{2}\right)}{\left(p-p_{3}\right)\left(q-p_{3}\right)}\right)^{\frac{1}{p_{2}-p_{3}}}\left(\frac{\left(p-p_{4}\right)\left(q-p_{4}\right)}{\left(p-p_{1}\right)\left(q-p_{1}\right)}\right)^{\frac{1}{p_{1}-p_{4}}},
$$

and in the third case we have

$$
u(p, q)=\left(\frac{\left(p-p_{3}\right)\left(q-p_{3}\right)}{\left(p-p_{1}\right)\left(q-p_{1}\right)}\right)^{\frac{1}{p_{1}-p_{3}}}\left(\frac{\left(p-p_{2}\right)\left(q-p_{2}\right)}{\left(p-p_{4}\right)\left(q-p_{4}\right)}\right)^{\frac{1}{p_{2}-p_{4}}} .
$$

Perhaps not surprisingly these changes of coordinates are very similar to those appearing in equation (25) of [53]. 


\section{C.5 General instanton on Plebanski-Demianski}

Here we illustrate the construction of the general one-parameter instanton, starting directly from the Plebanski-Demianski metric

$$
\mathrm{d} s_{\mathrm{PD}}^{2}=\frac{\mathcal{P}(q)}{q^{2}-p^{2}}\left(\mathrm{~d} \tau+p^{2} \mathrm{~d} \sigma\right)^{2}-\frac{\mathcal{P}(p)}{q^{2}-p^{2}}\left(\mathrm{~d} \tau+q^{2} \mathrm{~d} \sigma\right)^{2}+\frac{q^{2}-p^{2}}{\mathcal{P}(q)} \mathrm{d} q^{2}-\frac{q^{2}-p^{2}}{\mathcal{P}(p)} \mathrm{d} p^{2},
$$

with

$$
\mathcal{P}(x)=x^{4}+E x^{2}-2 M x+L,
$$

and a Killing vector

$$
K=b_{\tau} \partial_{\tau}+b_{\sigma} \partial_{\sigma},
$$

with generic coefficients $b_{\tau}, b_{\sigma}$. As before, we will denote the constant coefficient of the quartic $\mathcal{P}(x)$ with the symbol $L$, instead of " $-Q^{2}+\alpha$ ". This is to emphasize the fact that in our general set up the parameters $L, b_{\tau}, b_{\sigma}$ are independent.

Recall that given the one-form $K^{b}$, dual to $K$, and the expression for $y$ in (3.34), the instanton $F$ can be derived using the following formula

$$
F=-\left(\frac{1}{2} y \mathrm{~d} K^{b}+y^{2} K^{b} \wedge J K^{b}\right)^{-}
$$

where the complex structure tensor is

$$
J_{\nu}^{\mu}=-y g^{\mu \rho}\left(\mathrm{d} K^{b}\right)_{\rho \nu}^{+} .
$$

Here $g^{\mu \nu}$ is the inverse of the self-dual Einstein metric, and the contraction with the complex structure is defined as $J K^{b}=J^{\mu}{ }_{\nu} K_{\mu}^{b} \mathrm{~d} x^{\nu}$. We then obtain the following general expression

$$
\begin{aligned}
\frac{1}{y^{2}}=\frac{1}{4} \frac{1}{\left(q^{2}-p^{2}\right)^{2}}\{[ & {\left.\left[\frac{2 \mathcal{P}(q)}{q-p}-\mathcal{P}^{\prime}(q)\right)\left(b_{\tau}+b_{\sigma} p^{2}\right)-\left(\frac{2 \mathcal{P}(p)}{q-p}+\mathcal{P}^{\prime}(p)\right)\left(b_{\tau}+b_{\sigma} q^{2}\right)\right]^{2} } \\
& \left.-4 b_{\sigma}^{2} \mathcal{P}(q) \mathcal{P}(p)(q+p)^{2}\right\} .
\end{aligned}
$$

Inserting the polynomial $\mathcal{P}(x)$, we see that this is actually a polynomial of degree two, symmetric in $p$ and $q$, namely

$$
\begin{aligned}
\frac{1}{y(p, q)^{2}}= & p^{2} q^{2}\left(2 b_{\tau} b_{\sigma}-b_{\sigma}^{2} E\right)+2 p q\left(b_{\tau} b_{\sigma} E-b_{\tau}^{2}-b_{\sigma}^{2} L\right)+\left(p^{2}+q^{2}\right)\left(b_{\tau}^{2}-b_{\sigma}^{2} L\right) \\
& +2 b_{\sigma}^{2} M\left(p^{2} q+p q^{2}\right)-2 b_{\tau} b_{\sigma} M(p+q)+2 b_{\tau} b_{\sigma} L+b_{\sigma}^{2}\left(M^{2}-E L\right)
\end{aligned}
$$

In the frame

$$
\begin{aligned}
\tilde{e}^{1} & =\sqrt{\frac{q^{2}-p^{2}}{-\mathcal{P}(p)}} \mathrm{d} p, & \tilde{e}^{2} & =\sqrt{\frac{-\mathcal{P}(p)}{q^{2}-p^{2}}}\left(\mathrm{~d} \tau+q^{2} \mathrm{~d} \sigma\right), \\
\tilde{e}^{3} & =\sqrt{\frac{\mathcal{P}(q)}{q^{2}-p^{2}}}\left(\mathrm{~d} \tau+p^{2} \mathrm{~d} \sigma\right), & \tilde{e}^{4} & =\sqrt{\frac{q^{2}-p^{2}}{\mathcal{P}(q)}} \mathrm{d} q,
\end{aligned}
$$


the instanton takes the form

$$
\begin{aligned}
F= & \left(\tilde{e}^{13}+\tilde{e}^{24}\right) \frac{y(p, q)^{3} \sqrt{-\mathcal{P}(p) \mathcal{P}(q)}}{2(q+p)}\left(b_{\sigma}^{3}\left(M^{2}-E L\right)-2 b_{\tau}^{3}+b_{\tau}^{2} b_{\sigma} E+2 b_{\tau} b_{\sigma}^{2} L\right) \\
& +\left(\tilde{e}^{12}-\tilde{e}^{34}\right) \frac{y(p, q)^{3}}{32 Q^{8}(p+q)^{2}} \sum_{m, n=0}^{3} a_{m n} q^{m} p^{n}
\end{aligned}
$$

with symmetric coefficients, $a_{m n}=a_{n m}$, given by

$$
\begin{aligned}
& a_{00}=2 b_{\tau} b_{\sigma} M\left(2 b_{\tau} L+b_{\sigma}\left(M^{2}-E L\right)\right) \\
& a_{01}=-2 b_{\tau}^{3} L+b_{\tau}^{2} b_{\sigma}\left(E L-6 M^{2}\right)+2 b_{\tau} b_{\sigma}^{2} L^{2}+b_{\sigma}^{3} L\left(M^{2}-E L\right) \\
& a_{02}=M\left(4 b_{\tau}^{3}+b_{\tau}^{2} b_{\sigma} E-4 b_{\tau} b_{\sigma}^{2} L+b_{\sigma}^{3}\left(-E L+M^{2}\right)\right) \\
& a_{03}=-b_{\tau}^{3} E+2 b_{\tau}^{2} b_{\sigma} L+b_{\tau} b_{\sigma}^{2}\left(E L-M^{2}\right)-2 b_{\sigma}^{3} L^{2} \\
& a_{11}=6 b_{\tau} b_{\sigma} M\left(b_{\tau} E-2 b_{\sigma} L\right) \\
& a_{12}=-b_{\tau}^{3} E+b_{\tau}^{2} b_{\sigma}\left(6 L-E^{2}\right)+b_{\tau} b_{\sigma}^{2}\left(E L+9 M^{2}\right)+b_{\sigma}^{3}\left(E^{2} L-E M^{2}-6 L^{2}\right) \\
& a_{13}=6 b_{\sigma} M\left(b_{\sigma}^{2} L-b_{\tau}^{2}\right) \\
& a_{22}=6 b_{\sigma}^{2} M\left(2 b_{\sigma} L-b_{\tau} E\right) \\
& a_{23}=-2 b_{\tau}^{3}+b_{\tau}^{2} b_{\sigma} E+2 b_{\tau} b_{\sigma}^{2} L-b_{\sigma}^{3}\left(E L+5 M^{2}\right) \\
& a_{33}=2 b_{\sigma}^{2} M\left(b_{\sigma} E-2 b_{\tau}\right)
\end{aligned}
$$

These are all homogeneous degree three polynomials in the parameters $b_{\tau}, b_{\sigma}$, but only their ratio is important, so we could set one of them to unity. We can also express the instanton in terms of $b_{1}$ and $b_{2}$, using the relations

$$
\begin{aligned}
& b_{\tau}=\frac{2 p_{3}^{2}}{\mathcal{P}^{\prime}\left(p_{3}\right)} b_{1}-\frac{2 p_{4}^{2}}{\mathcal{P}^{\prime}\left(p_{4}\right)} b_{2}, \\
& b_{\sigma}=-\frac{2}{\mathcal{P}^{\prime}\left(p_{3}\right)} b_{1}+\frac{2}{\mathcal{P}^{\prime}\left(p_{4}\right)} b_{2} .
\end{aligned}
$$

The $\left(\tilde{e}^{13}+\tilde{e}^{24}\right)$ component is rather simple and reads

$$
\begin{aligned}
\left.F\right|_{\left(\tilde{e}^{13}+\tilde{e}^{24}\right)}= & \frac{y^{3}\left(p_{3}-p_{4}\right) \sqrt{-\mathcal{P}(p) \mathcal{P}(q)}}{(q+p) \mathcal{P}^{\prime}\left(p_{3}\right) \mathcal{P}^{\prime}\left(p_{4}\right)} \\
& \times\left(b_{1}+b_{2}\right)\left(b_{1}\left(p_{4}-p_{1}\right)-b_{2}\left(p_{3}-p_{2}\right)\right)\left(b_{1}\left(p_{4}-p_{2}\right)-b_{2}\left(p_{3}-p_{1}\right)\right),
\end{aligned}
$$

whereas the $\left(\tilde{e}^{12}-\tilde{e}^{34}\right)$ component does not simplify and we will not write it here.

Notice that the second line in (C.98) vanishes precisely in the three cases corresponding to the solutions in [10], where this part of the instanton is absent. These correspond precisely to the special choice

$$
\left.\frac{b_{\tau}}{b_{\sigma}}\right|_{\mathrm{MP}}=\frac{1}{2}\left(\frac{M^{2}}{Q^{2}}+E\right)
$$

Inserting this into (C.92) one finds that $1 / y^{2}$ factorizes, so that $1 / y$ becomes homogeneous of degree one in $p$ and $q$, as in (C.74). Similarly, the symmetric polynomial 
$\sum_{m, n=0}^{3} a_{m n} q^{m} p^{n}$ also becomes the cube of a degree one polynomial, so that the two functions cancel, leaving the enormously simplified instanton

$$
F=-\frac{Q}{(q+p)^{2}}\left(\tilde{e}^{12}-\tilde{e}^{34}\right),
$$

in agreement ${ }^{30}$ with $(2.28)$ of [10].

We also note that under the exchange of $p$ and $q$ the two terms transform as

$$
\begin{aligned}
& \left(\tilde{e}^{12}-e^{34}\right) \rightarrow\left(\tilde{e}^{12}-\tilde{e}^{34}\right), \\
& \left(\tilde{e}^{13}+e^{24}\right) \rightarrow-\left(\tilde{e}^{13}+\tilde{e}^{24}\right),
\end{aligned}
$$

respectively, while the functions entering in $F$ are all symmetric. Therefore, the special instantons in [10] are symmetric under this exchange, while the general instanton is neither symmetric nor antisymmetric, thus breaking this symmetry completely.

In conclusion, in this subsection we have explicitly shown how starting from a metric with two non-trivial parameters $(E, M, L \bmod$ scaling symmetry) we have obtained an instanton, and hence a full supersymmetric solution, depending on one further non-trivial parameter $\left(b_{\tau}, b_{\sigma}\right.$ modulo scaling symmetry). By contrast, in the construction of [10], the full solution depends on only two non-trivial parameters, already appearing in the Plebanski-Demianski metric, and the instanton does not introduce a new parameter due to the relation (C.99).

\section{C.6 Taub-NUT-AdS ${ }_{4}$ as a limit of Plebanski-Demianski}

Here we will show how to recover the Taub-NUT-AdS 4 metric

$$
\mathrm{d} s_{4}^{2}=\frac{r^{2}-s^{2}}{\Omega(r)} \mathrm{d} r^{2}+\left(r^{2}-s^{2}\right)\left(\mathrm{d} \theta^{2}+\sin ^{2} \theta \mathrm{d} \varphi^{2}\right)+\frac{4 s^{2} \Omega(r)}{r^{2}-s^{2}}(\mathrm{~d} \varsigma+\cos \theta \mathrm{d} \varphi)^{2},
$$

with

$$
\Omega(r)=(r-s)^{2}(1+(r-s)(r+3 s)),
$$

from a limit of the Plebanski-Deminaski metric (C.58), thus demonstrating that the former is a one-parameter sub-family of the toric 3-pole metric, where the isometry enhances to $\mathrm{SU}(2) \times \mathrm{U}(1)$. Applying the same limit to the general instanton on the Plebanski-Demianski metric (C.94) we will also obtain an explicit expression for the general toric instanton on the Taub-NUT-AdS 4 metric.

Following [10] we parameterise the four roots of $\mathcal{P}(x)$ in terms of two constants $\hat{a}, s$ as

$$
\begin{array}{llrl}
p_{1} & =-\frac{1}{2}-\sqrt{\hat{a}^{2}-2 M}, & p_{2} & =-\frac{1}{2}+\sqrt{\hat{a}^{2}-2 M}, \\
p_{3} & =\frac{1}{2}-\hat{a}, & p_{4} & =\frac{1}{2}+\hat{a},
\end{array}
$$

with $^{31}$

$$
2 M=\frac{1}{4 s^{2}}-1
$$

\footnotetext{
${ }^{30} \mathrm{Up}$ to an overall sign related to charge conjugation of the spinor — see the discussion in the paragraph before equation (2.8).

${ }^{31}$ In [10] the squashing parameter $s$ was denoted $\frac{1}{2 v}$.
} 
Then we make the following change of coordinates

$$
p=\frac{1}{2}-\hat{a} \cos \theta, \quad q=\frac{r}{2 s},
$$

and

$$
\tau=-\left(4 s^{2}+\frac{k}{4}\right) \varsigma-\frac{s^{2}}{\hat{a}} \varphi, \quad \sigma=k \varsigma+\frac{4 s^{2}}{\hat{a}} \varphi,
$$

with $k$ an arbitrary real number. Substituting $p, q, \tau, \sigma$ above into the Plebanski-Demianski metric and taking the limit $\hat{a} \rightarrow 0$, it is straightforward to verify that one obtains precisely the Taub-NUT-AdS 4 metric (C.102).

Comparing the expression of the Killing vector in section C.5, namely

$$
K=\partial_{\psi}=b_{\tau} \partial_{\tau}+b_{\sigma} \partial_{\sigma}
$$

with that given in section 5.2, namely

$$
K=\left(b_{1}+b_{2}\right) \partial_{\varphi}+\left(b_{1}-b_{2}\right) \partial_{\varsigma}
$$

we deduce that the parameters $b_{\tau}, b_{\sigma}$ must be related to $b_{1}, b_{2}$ as

$$
b_{\tau}=-\frac{s^{2}}{\hat{a}}\left(b_{1}+b_{2}\right)+\left(\frac{k}{4}+4 s^{2}\right)\left(b_{2}-b_{1}\right), \quad b_{\sigma}=\frac{4 s^{2}}{\hat{a}}\left(b_{1}+b_{2}\right)+k\left(b_{1}-b_{2}\right) .
$$

Inserting these into the expression for $y(p, q)$ in (C.92) along with (C.106) and (C.107), and then taking the limit $\hat{a} \rightarrow 0$, one finds precisely the $y(r, \theta)$ given in (5.13). Notice that the final result does not depend on $k$.

Finally, using this change of coordinate/parameters in the instanton (C.94), we find the following explicit expression for general instanton on the Taub-NUT-AdS 4 metric

$$
\begin{aligned}
F= & \frac{y^{3}}{2}\left(b_{1}+b_{2}\right)\left(16 b_{1} b_{2} s^{2}-\left(b_{1}+b_{2}\right)^{2}\right)(r-s) \sin \theta\left(\frac{2 s \Omega(r)}{r^{2}-s^{2}} \mathrm{~d} \theta \wedge \tau_{3}+\sin \theta \mathrm{d} r \wedge \mathrm{d} \varphi\right) \\
& +\frac{y^{3}(r-s)^{2}}{2(r+s)}\left(\frac{2 s}{r^{2}-s^{2}} \mathrm{~d} r \wedge \tau_{3}-\tau_{1} \wedge \tau_{2}\right) \\
& \times\left(-\left(b_{1}+b_{2}\right)^{2} \sin ^{2} \theta\left[\left(4 s^{2}-1\right)\left(b_{1}+b_{2}\right)\left(4 r^{2} s+4 r s^{2}+r-8 s^{3}+3 s\right) \cos \theta\right.\right. \\
& \left.\quad+2 s\left(b_{1}-b_{2}\right)\left(r^{2}\left(8 s^{2}-1\right)+2 s r\left(4 s^{2}+1\right)-16 s^{4}+11 s^{2}+\frac{2 s}{r-s}\right)\right] \\
& +(2 s(r-s)+1)\left(4 s^{2}-1\right)\left[s\left(4 s^{2}-1\right)-r\left(4 s^{2}+1\right)-2 s\right]\left(b_{1}+b_{2}\right)^{3} \cos ^{3} \theta \\
& -2 s\left(b_{1}-b_{2}\right)\left(b_{1}+b_{2}\right)^{2} \cos ^{2} \theta \\
& \times\left(r^{2}\left(48 s^{4}-4 s^{2}-1\right)-4 r\left(24 s^{5}-14 s^{3}+s\right)+s^{2}\left(48 s^{4}-52 s^{2}+17\right)+\frac{2 s}{r-s}\right) \\
& -8 s^{3}\left(b_{1}-b_{2}\right)^{2}\left(b_{1}+b_{2}\right) \cos \theta\left(1+2(r-s)\left(6 r s^{2}-r-6 s^{3}\right)+\frac{r-s}{2 s}\left(16 s^{2}-1\right)\right) \\
& \left.-8 s^{3}\left(b_{1}-b_{2}\right)^{3}(r-s)^{2}\left(4 s^{2}-1\right)\right),
\end{aligned}
$$


where $y(r, \theta)$ is given by (5.13) and $\tau_{i}$ are the $\mathrm{SU}(2)$ left-invariant one-forms

$$
\begin{aligned}
& \tau_{1}=\cos \varsigma \mathrm{d} \theta+\sin \varsigma \sin \theta \mathrm{d} \varphi, \\
& \tau_{2}=-\sin \varsigma \mathrm{d} \theta+\cos \varsigma \sin \theta \mathrm{d} \varphi, \\
& \tau_{3}=\mathrm{d} \varsigma+\cos \theta \mathrm{d} \varphi .
\end{aligned}
$$

Indeed, for $b_{1}=-b_{2}$ this reduces to the $1 / 4$-BPS instanton in $(5.20)$

$$
F_{\frac{1}{4} \mathrm{BPS}}=\frac{1}{2}\left(4 s^{2}-1\right)\left(\frac{2 s}{(r+s)^{2}} \mathrm{~d} r \wedge \tau_{3}-\frac{r-s}{r+s} \tau_{1} \wedge \tau_{2}\right),
$$

up to a sign related to charge conjugation of the spinor. While for

$$
b_{1}=\frac{1}{4 s}, \quad b_{2}=-\frac{1}{4 s}+2 s+\sqrt{4 s^{2}-1},
$$

it reduces to the $1 / 2$-BPS instanton in (5.24)

$$
F_{\frac{1}{2} \mathrm{BPS}}=s \sqrt{4 s^{2}-1}\left(\frac{2 s}{(r+s)^{2}} \mathrm{~d} r \wedge \tau_{3}-\frac{r-s}{r+s} \tau_{1} \wedge \tau_{2}\right),
$$

again up to a sign related to charge conjugation.

Finally, taking the limit $r \rightarrow \infty$ of (C.111), it is straightforward to extract the background gauge field induced on the boundary. This has field strength

$$
\begin{aligned}
F_{(0)}= & \frac{\sqrt{2} s}{\mathcal{X}^{3 / 2}}\left[\tau _ { 1 } \wedge \tau _ { 2 } \left(\left(4 s^{2}-1\right)^{2} b_{+}^{3} \cos ^{3} \theta+12 s^{2}\left(4 s^{2}-1\right) b_{-} b_{+}^{2} \cos ^{2} \theta\right.\right. \\
& \left.+2\left(24 s^{4} b_{-}^{2}+16 s^{2} b_{1} b_{2}-b_{+}^{2}\right) b_{+} \cos \theta+\left(8 s^{2}-1\right) b_{-} b_{+}^{2}+4 s^{2}\left(4 s^{2}-1\right) b_{-}^{3}\right) \\
& \left.+b_{+}\left(16 s^{2} b_{1} b_{2}-b_{+}^{2}\right) \sin \theta \mathrm{d} \theta \wedge \tau_{3}\right],
\end{aligned}
$$

where we defined $b_{ \pm} \equiv b_{1} \pm b_{2}$ and

$$
\mathcal{X}=b_{+}^{2} \sin ^{2} \theta+4 s^{2}\left(b_{-}+b_{+} \cos \theta\right)^{2} .
$$

The corresponding gauge field takes the form

$$
A_{(0)}^{\text {local }}=f_{\varphi}(\theta) \mathrm{d} \varphi+f_{\varsigma}(\theta) \mathrm{d} \varsigma,
$$

where

$$
\begin{aligned}
f_{\varphi}(\theta) & =\frac{s}{\sqrt{\mathcal{X}}}\left(b_{+}-\left(4 s^{2}-1\right)\left(b_{-}+b_{+} \cos \theta\right) \cos \theta\right) \\
f_{\varsigma}(\theta) & =-\frac{s}{\sqrt{\mathcal{X}}}\left(4 s^{2} b_{-}+\left(4 s^{2}-1\right) b_{+} \cos \theta\right)
\end{aligned}
$$

This provides an explicit one-parameter family of three-dimensional backgrounds interpolating between those of [7] and [21]. Of course, in general this preserves only a $\mathrm{U}(1) \times \mathrm{U}(1)$ subgroup of the isometry group of the biaxially squashed sphere, which is enhanced to $\mathrm{SU}(2) \times \mathrm{U}(1)$ in the two special cases above. 
Open Access. This article is distributed under the terms of the Creative Commons Attribution License (CC-BY 4.0), which permits any use, distribution and reproduction in any medium, provided the original author(s) and source are credited.

\section{References}

[1] J.M. Maldacena, The large- $N$ limit of superconformal field theories and supergravity, Int. J. Theor. Phys. 38 (1999) 1113 [hep-th/9711200] [INSPIRE].

[2] S.S. Gubser, I.R. Klebanov and A.M. Polyakov, Gauge theory correlators from noncritical string theory, Phys. Lett. B 428 (1998) 105 [hep-th/9802109] [INSPIRE].

[3] E. Witten, Anti-de Sitter space and holography, Adv. Theor. Math. Phys. 2 (1998) 253 [hep-th/9802150] [INSPIRE].

[4] V. Pestun, Localization of gauge theory on a four-sphere and supersymmetric Wilson loops, Commun. Math. Phys. 313 (2012) 71 [arXiv:0712.2824] [inSPIRE].

[5] G. Festuccia and N. Seiberg, Rigid supersymmetric theories in curved superspace, JHEP 06 (2011) 114 [arXiv:1105.0689] [INSPIRE].

[6] D. Martelli, A. Passias and J. Sparks, The gravity dual of supersymmetric gauge theories on a squashed three-sphere, Nucl. Phys. B 864 (2012) 840 [arXiv:1110.6400] [INSPIRE].

[7] N. Hama, K. Hosomichi and S. Lee, SUSY gauge theories on squashed three-spheres, JHEP 05 (2011) 014 [arXiv: 1102.4716] [INSPIRE].

[8] D. Martelli and J. Sparks, The gravity dual of supersymmetric gauge theories on a biaxially squashed three-sphere, Nucl. Phys. B 866 (2013) 72 [arXiv:1111.6930] [INSPIRE].

[9] D. Martelli, A. Passias and J. Sparks, The supersymmetric NUTs and bolts of holography, Nucl. Phys. B 876 (2013) 810 [arXiv:1212.4618] [INSPIRE].

[10] D. Martelli and A. Passias, The gravity dual of supersymmetric gauge theories on a two-parameter deformed three-sphere, Nucl. Phys. B 877 (2013) 51 [arXiv:1306.3893] [INSPIRE].

[11] X. Huang, S.-J. Rey and Y. Zhou, Three-dimensional SCFT on conic space as hologram of charged topological black hole, JHEP 03 (2014) 127 [arXiv:1401.5421] [INSPIRE].

[12] T. Nishioka, The gravity dual of supersymmetric Rényi entropy, JHEP 07 (2014) 061 [arXiv: 1401.6764] [INSPIRE].

[13] T. Nishioka and I. Yaakov, Supersymmetric Rényi entropy, JHEP 10 (2013) 155 [arXiv:1306.2958] [INSPIRE].

[14] D. Cassani and D. Martelli, The gravity dual of supersymmetric gauge theories on a squashed $S^{1} \times S^{3}$, JHEP 08 (2014) 044 [arXiv: 1402.2278] [INSPIRE].

[15] L.F. Alday, M. Fluder, P. Richmond and J. Sparks, Gravity dual of supersymmetric gauge theories on a squashed five-sphere, Phys. Rev. Lett. 113 (2014) 141601 [arXiv:1404.1925] [INSPIRE].

[16] D.Z. Freedman and S.S. Pufu, The holography of F-maximization, JHEP 03 (2014) 135 [arXiv: 1302.7310] [INSPIRE].

[17] J.G. Russo and K. Zarembo, Large- $N$ limit of $N=2 \mathrm{SU}(N)$ gauge theories from localization, JHEP 10 (2012) 082 [arXiv: 1207.3806] [INSPIRE]. 
[18] A. Buchel, J.G. Russo and K. Zarembo, Rigorous test of non-conformal holography: Wilson loops in $N=2^{*}$ theory, JHEP 03 (2013) 062 [arXiv:1301.1597] [INSPIRE].

[19] N. Bobev, H. Elvang, D.Z. Freedman and S.S. Pufu, Holography for $N=2^{*}$ on $S^{4}$, JHEP 07 (2014) 001 [arXiv:1311.1508] [INSPIRE].

[20] L.F. Alday, D. Martelli, P. Richmond and J. Sparks, Localization on three-manifolds, JHEP 10 (2013) 095 [arXiv: 1307.6848] [INSPIRE].

[21] Y. Imamura and D. Yokoyama, $N=2$ supersymmetric theories on squashed three-sphere, Phys. Rev. D 85 (2012) 025015 [arXiv:1109.4734] [inSPIRE].

[22] C. Closset, T.T. Dumitrescu, G. Festuccia and Z. Komargodski, The geometry of supersymmetric partition functions, JHEP 01 (2014) 124 [arXiv:1309.5876] [INSPIRE].

[23] N. Drukker, M. Mariño and P. Putrov, From weak to strong coupling in ABJM theory, Commun. Math. Phys. 306 (2011) 511 [arXiv:1007.3837] [InSPIRE].

[24] C. Klare, A. Tomasiello and A. Zaffaroni, Supersymmetry on curved spaces and holography, JHEP 08 (2012) 061 [arXiv:1205.1062] [INSPIRE].

[25] C. Closset, T.T. Dumitrescu, G. Festuccia and Z. Komargodski, Supersymmetric field theories on three-manifolds, JHEP 05 (2013) 017 [arXiv:1212.3388] [INSPIRE].

[26] D.M.J. Calderbank and H. Pedersen, Selfdual Einstein metrics with torus symmetry, J. Diff. Geom. 60 (2002) 485 [math/0105263] [INSPIRE].

[27] D.Z. Freedman and A.K. Das, Gauge internal symmetry in extended supergravity, Nucl. Phys. B 120 (1977) 221 [INSPIRE].

[28] M. Dunajski, J.B. Gutowski, W.A. Sabra and P. Tod, Cosmological Einstein-Maxwell instantons and Euclidean supersymmetry: beyond self-duality, JHEP 03 (2011) 131 [arXiv: 1012.1326] [INSPIRE].

[29] M. Dunajski, J. Gutowski, W. Sabra and P. Tod, Cosmological Einstein-Maxwell instantons and Euclidean supersymmetry: anti-self-dual solutions, Class. Quant. Grav. 28 (2011) 025007 [arXiv: 1006 . 5149] [INSPIRE].

[30] C. Lebrun, On complete quaternionic Kahler manifolds, Duke Math. J. 63 (1991) 723.

[31] K.P. Tod, The SU( $\infty$ )-Toda field equation and special four-dimensional metrics, in Geometry and physics, Aarhus Denmark (1995), pg. 307 and in Lecture Notes in Pure and Appl. Math. 184, Dekker, New York U.S.A. (1997) [INSPIRE].

[32] J.P. Gauntlett, D. Martelli and D. Waldram, Superstrings with intrinsic torsion, Phys. Rev. D 69 (2004) 086002 [hep-th/0302158] [INSPIRE].

[33] D. Martelli, J. Sparks and S.-T. Yau, Sasaki-Einstein manifolds and volume minimisation, Commun. Math. Phys. 280 (2008) 611 [hep-th/0603021] [INSPIRE].

[34] R. Emparan, C.V. Johnson and R.C. Myers, Surface terms as counterterms in the AdS/CFT correspondence, Phys. Rev. D 60 (1999) 104001 [hep-th/9903238] [INSPIRE].

[35] K. Skenderis, Lecture notes on holographic renormalization, Class. Quant. Grav. 19 (2002) 5849 [hep-th/0209067] [INSPIRE].

[36] M.T. Anderson, $L^{2}$ curvature and volume renormalization of AHE metrics on 4-manifolds, Math. Res. Lett. 8 (2001) 171 [math/0011051]. 
[37] M.F. Atiyah, V.K. Patodi and I.M. Singer, Spectral asymmetry and Riemannian geometry, I, Math. Proc. Camb. Philos. Soc. 77 (1975) 43.

[38] N.J. Hitchin, Einstein metrics and the eta-invariant, Boll. Union. Mat. Ital. B 11 (1997) 95.

[39] M.T. Anderson, Geometric aspects of the AdS/CFT correspondence, IRMA Lect. Math. Theor. Phys. 8 (2005) 1 [hep-th/0403087] [INSPIRE].

[40] N. Hitchin, Harmonic spinors, Adv. Math. 14 (1974) 1.

[41] J.F. Plebanski and M. Demianski, Rotating, charged and uniformly accelerating mass in general relativity, Annals Phys. 98 (1976) 98 [INSPIRE].

[42] O. Biquard, Metriques autoduales sur la boule (in French), Preprint IRMA, Strasbourg France (2000) [math/0010188].

[43] C. Fefferman and C.R. Graham, Conformal invariants, in The Mathematical Heritage of Elie Cartan, Lyon (1984), Asterisque, France (1985), pg. 95.

[44] M.T. Anderson, On the structure of conformally compact Einstein metrics, math/0402198.

[45] D.M.J. Calderbank and M.A. Singer, Einstein metrics and complex singularities, Invent. Math. 156 (2004) 405 [math/0206229] [INSPIRE].

[46] D. Martelli, J. Sparks and S.-T. Yau, The geometric dual of a-maximisation for toric Sasaki-Einstein manifolds, Commun. Math. Phys. 268 (2006) 39 [hep-th/0503183] [INSPIRE].

[47] A. Butti and A. Zaffaroni, R-charges from toric diagrams and the equivalence of a-maximization and Z-minimization, JHEP 11 (2005) 019 [hep-th/0506232] [INSPIRE].

[48] D. Martelli and J. Sparks, The large-N limit of quiver matrix models and Sasaki-Einstein manifolds, Phys. Rev. D 84 (2011) 046008 [arXiv:1102.5289] [InSPIRE].

[49] J.P. Gauntlett and O. Varela, Consistent Kaluza-Klein reductions for general supersymmetric AdS solutions, Phys. Rev. D 76 (2007) 126007 [arXiv:0707.2315] [InSPIRE].

[50] D. Farquet and J. Sparks, Wilson loops on three-manifolds and their M2-brane duals, JHEP 12 (2014) 173 [arXiv: 1406.2493] [INSPIRE].

[51] D. Farquet and J. Sparks, Wilson loops and the geometry of matrix models in AdS $S_{4} / C_{F T}$, JHEP 01 (2014) 083 [arXiv: 1304.0784] [INSPIRE].

[52] V. Apostolov, D.M.J. Calderbank and P. Gauduchon, The geometry of weakly selfdual Kähler surfaces, Composit. Math. 135 (2003) 279 [math/0104233].

[53] D. Martelli and J. Sparks, Toric Sasaki-Einstein metrics on $S^{2} \times S^{3}$, Phys. Lett. B 621 (2005) 208 [hep-th/0505027] [INSPIRE]. 